\title{
TREE-AUTOMATIC WELL-FOUNDED TREES *
}

\author{
MARTIN HUSCHENBETT ${ }^{a}$, ALEXANDER KARTZOW $^{b}$, JIAMOU LIU $^{c}$, AND MARKUS LOHREY ${ }^{d}$ \\ ${ }^{a}$ Technische Universität Ilmenau, Germany \\ e-mail address: martin.huschenbett@tu-ilmenau.de \\ ${ }^{b, d}$ Universität Leipzig, Germany \\ e-mail address: \{kartzow, lohrey\}@informatik.uni-leipzig.de \\ ${ }^{c}$ Auckland University of Technology, New Zealand \\ e-mail address: jiamou.liu@aut.ac.nz
}

\begin{abstract}
We investigate tree-automatic well-founded trees. Using Delhommé's decomposition technique for tree-automatic structures, we show that the (ordinal) rank of a tree-automatic well-founded tree is strictly below $\omega^{\omega}$. Moreover, we make a step towards proving that the ranks of tree-automatic well-founded partial orders are bounded by $\omega^{\omega^{\omega}}$ : we prove this bound for what we call upwards linear partial orders. As an application of our result, we show that the isomorphism problem for tree-automatic well-founded trees is complete for level $\Delta_{\omega \omega}^{0}$ of the hyperarithmetical hierarchy with respect to Turingreductions.
\end{abstract}

\section{INTRODUCTION}

Various classes of infinite but finitely presented structures received a lot of attention in algorithmic model theory [2]. Among the most important such classes of structures is the class of string-automatic structures [17]. A (relational) structure is string-automatic if its universe is a regular set of words and all relations can be recognized by synchronous multi-tape automata. During the past 15 years a theory of string-automatic structures has emerged. This theory was developed along two interrelated branches. The first is a structural branch, which leads to (partial) characterizations of particular classes of stringautomatic structures [9, 16, 18, 19, 22. The second is an algorithmic branch, which leads to numerous decidability and undecidability, as well as complexity results for important algorithmic problems for string-automatic structures [4, 18, 21]. One of the most fundamental

2012 ACM CCS: [Theory of computation]: Logic; Formal languages and automata theory-Tree languages.

Key words and phrases: hyperarithmetical hierarchy, isomorphism problem, ordinal rank, tree-automatic structures, well-founded trees.

* An extended abstract of this paper appeared at CiE 2012 [14].

${ }^{b, d}$ The second and fourth author are supported by the DFG research project GELO.

넴
DOI:10.2168/LMCS-9(2:10)2013

(C) M. Huschenbett, A. Kartzow, J. Liu, and M. Lohrey Creative Commons 
results for string-automatic structures states that their first-order theories are uniformly decidable [17.

By replacing strings and string automata by trees and tree automata, respectively, Blumensath [3] generalized string-automatic structures to tree-automatic structures and proved that their first-order theories are still uniformly decidable. However compared to string-automatic structures, the theory of tree-automatic structures is less developed. The only non-trivial characterization of a class of tree-automatic structures we are aware of concerns ordinals. In [9] Delhommé proved that an ordinal is tree-automatic if and only if it is strictly below $\omega^{\omega^{\omega}}$. Some complexity results for first-order theories of tree-automatic structures are shown in [21]. Recently, Huschenbett proved that it is decidable whether a given tree-automatic scattered linear order is string-automatic [12.

In this paper, we study tree-automatic well-founded trees. We stress here that in this paper the term tree always refers to order trees $\mathfrak{T}=(T, \leq)$, i.e., partial orders that have the shape of a tree, as opposed to successor trees where the edge relation is not transitive (except for trivial cases). Using Delhommé's decomposition technique for tree-automatic structures [9], we show that the rank of a tree-automatic well-founded tree is strictly below $\omega^{\omega}$. As a generalization of trees, we introduce the class of upwards linear partial orders. These are orders where the elements above any fixed element form a linear suborder. If such an order is well-founded and tree-automatic, then its rank is strictly below $\omega^{\omega^{\omega}}$. Unfortunately, we do not know if this bound holds for all tree-automatic well-founded partial orders. We will show in Example 3.27 that there is no hope to extend Delhommé's technique in order to obtain bounds on the ranks of all tree-automatic well-founded partial orders. Thus, characterizing the ranks of well-founded partial orders requires the development of completely new techniques.

We apply this result to the isomorphism problem for tree-automatic well-founded trees. In [20], it was shown that the isomorphism problem for string-automatic well-founded trees is complete for level $\Delta_{\omega}^{0}$ of the hyperarithmetical hierarchy. In other words, the isomorphism problem for string-automatic well-founded trees is recursively equivalent to true arithmetic. We show that the rank of well-founded computable trees determines the complexity of the isomorphism problem in the following sense: The isomorphism problem for well-founded computable trees of rank at most $\lambda$ (where $\lambda$ is a computable limit ordinal) belongs to level $\Delta_{\lambda}^{0}$ of the hyperarithmetical hierarchy. Since we know that the rank of a tree-automatic well-founded tree is strictly below $\omega^{\omega}$, this implies that the isomorphism problem for treeautomatic well-founded trees belongs to level $\Delta_{\omega^{\omega}}^{0}$ of the hyperarithmetical hierarchy. We also provide a corresponding lower bound. We prove that the isomorphism problem for tree-automatic well-founded trees is $\Delta_{\omega^{\omega}}^{0}$-complete under Turing-reductions.

Let us remark that for non-well-founded order trees, the isomorphism problem is complete for $\Sigma_{1}^{1}$ (the first existential level of the analytical hierarchy) already in the stringautomatic case [20], and this complexity is in a certain sense maximal, since the isomorphism problem for the class of all computable structures is $\Sigma_{1}^{1}$-complete as well [6, 10]. Let us also emphasize that our proof techniques do not work for successor trees.

\section{Preliminaries}

We write $\mathbb{N}_{>0}$ for $\mathbb{N} \backslash\{0\}$. For $M, N$ sets, $2^{M}$ denotes the powerset of $M$ and $N^{M}$ denotes the set of functions from $M$ to $N$. We denote by $M \sqcup N$ (by $\bigsqcup_{i \in N} M_{i}$, respectively) the 
disjoint union of the sets $M$ and $N\left(\left(M_{i}\right)_{i \in N}\right.$, respectively). For any (partial) function $f$ we write $\operatorname{dom}(f)$ for the domain of $f$.

A (finite and relational) signature $\tau=(\mathcal{R}$, ar) is a finite set $\mathcal{R}$ of relation symbols together with a map ar : $\mathcal{R} \rightarrow \mathbb{N}_{>0}$ assigning to each each $R \in \mathcal{R}$ its arity $\operatorname{ar}(R)$. A $\tau$ structure $\mathfrak{S}=\left(S,\left(R^{\mathfrak{S}}\right)_{R \in \mathcal{R}}\right)$ consists of a domain $S$ and an $\operatorname{ar}(R)$-ary relation $R^{\mathfrak{S}}$ on $S$ for each $R \in \mathcal{R}$. For $T \subseteq S$, we denote the substructure of $\mathfrak{S}$ induced by the restriction of $\mathfrak{S}$ to the set $T$ as $\left.\mathfrak{S}\right|_{T}$. In this paper we consider only structures with countable domains.

A partial order $\mathfrak{A}=(A, \leq)$ is regarded as a structure over a signature consisting of a single binary relation symbol $\leq$. Every substructure of $\mathfrak{A}$ is again a partial order. We call a subset $B \subseteq A$ an antichain if for all distinct $a, b \in B$, neither $a \leq b$ nor $b \leq a$.

Let $A$ be a (not necessarily finite) set. We use $\preceq$ to denote the prefix order on finite words in $A^{*}$, i.e., for $u, v \in A^{*}, u \preceq v$ if $v=u w$ for some $w \in A^{*}$. For a language $L \subseteq A^{*}$, let $\operatorname{pref}(L)=\left\{w \in A^{*} \mid \exists u \in L: w \preceq u\right\}$ be its prefix-closure.

2.1. Trees, forests and upwards linear partial orders. Let $\mathfrak{P}=(P, \leq)$ be a partial order. We say that $\mathfrak{P}$ is connected if the undirected graph $(P,\{(a, b) \mid a \leq b$ or $b \leq a\})$ is connected. For an element $p \in P$ let $\uparrow p=\{a \in P \mid p<a\}$ be its (strict) upwards closure and $\downarrow p=\{a \in P \mid a<p\}$ be its (strict) downwards closure. Two elements $a, b \in P$ are comparable if $a \leq b$ or $b \leq a$. We say that $\mathfrak{P}$ is upwards linear if for every $p \in P, \uparrow p$ induces a linear order $\mathfrak{P} \uparrow_{\uparrow p}$. A forest is an upwards linear partial order $\mathfrak{F}=(F, \leq)$ such that for every $p \in P$ the set $\uparrow p$ induces a finite linear order. A tree is a forest which has a greatest element, which is called the root of the tree. Note that a forest is a disjoint union of (an arbitrary number of) trees and all its substructures are also forests. For a given forest $\mathfrak{F}$, we denote as $\langle\mathfrak{F}\rangle$ the tree that results from adding a new root (i.e., a new greatest element) to $\mathfrak{F}$. If $F$ is the domain of $\mathfrak{F}$ we write $\langle F\rangle$ for the domain of $\langle\mathfrak{F}\rangle$. For a node $u$ in $\mathfrak{F}, \mathfrak{F}(u)$ denotes the subtree of $\mathfrak{F}$ at $u$, i.e., $\mathfrak{F}(u)=\mathfrak{F} \uparrow_{\{v \in F \mid v \leq u\}}$. We define the successor relation of $\mathfrak{F}$ as

$$
E_{\mathfrak{F}}=\left\{(x, y) \in F^{2} \mid x>y, \neg \exists z: x>z>y\right\} .
$$

For $x \in F$ the set of children of $x$ in $\mathfrak{F}$ is $E_{\mathfrak{F}}(x)=\left\{y \in F \mid(x, y) \in E_{\mathfrak{F}}\right\}$. The set of leaves of $\mathfrak{F}$ is leaves $(\mathfrak{F})=\left\{x \in F \mid E_{\mathfrak{F}}(x)=\emptyset\right\}$. A partial order $\mathfrak{P}=(P, \leq)$ is well-founded, if it does not contain an infinite descending chain $a_{1}>a_{2}>a_{3}>\cdots$. The sum of two partial orders $\mathfrak{P}_{1}=\left(P_{1}, \leq_{1}\right)$ and $\mathfrak{P}_{2}=\left(P_{2}, \leq_{2}\right)$ with $P_{1} \cap P_{2}=\emptyset$ is the partial order $\mathfrak{P}_{1}+\mathfrak{P}_{2}=\left(P_{1} \cup P_{2}, \leq\right)$, where $a \leq b$ if either $\left(a, b \in P_{1}\right.$ and $\left.a \leq_{1} b\right)$, or $\left(a, b \in P_{2}\right.$ and $\left.a \leq_{2} b\right)$, or $\left(a \in P_{1}\right.$ and $\left.b \in P_{2}\right)$.

We write wulpo as an abbreviation for well-founded upwards linear partial order. Note that the class of wulpo's contains all ordinals and all well-founded forests and is closed under taking substructures.

Let $\mathfrak{P}=(P, \leq)$ be some partial order. In the following, we set $p<\infty$ for all $p \in P$. In particular, $\infty$ is comparable to every $p \in P \cup\{\infty\}$ and $\max (p, \infty)=\infty$ for every $p \in P \cup\{\infty\}$. For $p_{1} \in P$ and $p_{2} \in P \cup\{\infty\}$ with $p_{1} \leq p_{2}$, we denote by $\left[p_{1}, p_{2}\right)$ the interval $\left\{p \in P \mid p_{1} \leq p<p_{2}\right\}$. We call this interval branching free if for all $p \in\left[p_{1}, p_{2}\right)$ we have

$$
\downarrow p=\downarrow p_{1}+\left[p_{1}, p\right) .
$$

In other words: $\left[p_{1}, p_{2}\right)$ is branching free, if for every $p \in\left[p_{1}, p_{2}\right)$ and every $x<p$, the points $x$ and $p_{1}$ are comparable. 
Lemma 2.1. Let $p_{1} \in P$ and $p_{2} \in P \cup\{\infty\}$ with $p_{1} \leq p_{2}$. If $\left[p_{1}, p_{2}\right)$ is branching free, then for every $p \in\left[p_{1}, p_{2}\right)$ also $\left[p_{1}, p\right)$ is branching free.

Proof. Assume that $x<p^{\prime} \in\left[p_{1}, p\right)$. We have to show that $x$ and $p_{1}$ are comparable. But this clear, since also $x<p^{\prime} \in\left[p_{1}, p_{2}\right)$ and the latter interval is branching free.

Lemma 2.2. Let $\mathfrak{P}=(P, \leq)$ be an upwards linear partial order and let $a, c \in P$ and $b, d \in P \cup\{\infty\}$ with $a \leq b$ and $c \leq d$. If $[a, b)$ and $[c, d)$ are branching free and not disjoint, then $a$ and $c$ are comparable, and $b$ and $d$ are comparable.

Proof. Assume that $[a, b)$ and $[c, d)$ are not disjoint and let $p \in[a, b) \cap[c, d)$. Since $\mathfrak{P}$ is upwards linear, the assumptions $p<b$ and $p<d$ imply that $b$ and $d$ are comparable. Moreover, since $c \leq p \in[a, b)$ and $[a, b)$ is branching free, the elements $c$ and $a$ are comparable too.

We call a branching free interval maximal branching free if it is maximal with respect to set inclusion. We use this interval notation mostly for upwards linear partial orders. In this case every interval induces a linear suborder.

Lemma 2.3. Let $\mathfrak{P}=(P, \leq)$ be an upwards linear partial order and let $a, c \in P$ and $b, d \in P \cup\{\infty\}$ with $a \leq b$ and $c \leq d$. If $[a, b)$ and $[c, d)$ are maximal branching free, then they are either disjoint or equal.

Proof. If $[a, b)$ and $[c, d)$ are not disjoint, Lemma 2.2 implies that $\max (b, d)$ and $\min (a, c)$ are defined. We claim that $[\min (a, c), \max (b, d))$ is also branching free. Since we assumed $[a, b)$ and $[c, d)$ to be maximal branching free, this implies $[a, b)=[\min (a, c), \max (b, d))=[c, d)$.

Let us now prove the claim. If $a=c$ or $b=d$ or $(a<c, d<b)$ or $(c<a, b<d)$ then $[\min (a, c), \max (b, d))$ is $[a, b)$ or $[c, d)$ and hence branching free. The remaining two cases are symmetric. Hence we can assume that $a<c$ and $b<d$ and we have to show that $[a, d)$ is branching free. Since $a \leq b$ and $a \leq c$, we have either $b \leq c$ or $c<b$. If $b \leq c$, then the intervals $[a, b)$ and $[c, d)$ would be disjoint. Hence we have

$$
a<c<b<d .
$$

To show that $[a, d)$ is branching free, let $x \in[a, d)$ and $y<x$. We have to show that $a$ and $y$ are comparable.

If $x \in[a, b)$ then $a$ and $y$ are comparable since $[a, b)$ is branching free. So, assume that $x<b$ does not hold. Since $\mathfrak{P}$ is upwards linear and $a \leq b, a \leq x$, it follows $b \leq x$. In particular $x \in[c, d)$. Since $[c, d)$ is branching free, $c$ and $y$ must be comparable. If $c \leq y$ then also $a \leq y$. Hence $y<c$. Since $c \in[a, b)$ and $[a, b)$ is branching free, $y$ and $a$ are comparable.

Lemma 2.4. Let $\mathfrak{P}$ be a wulpo. Every node of $\mathfrak{P}$ is contained in a unique maximal branching free interval.

Proof. Fix a node $p \in P$. Firstly, we define a node $p_{+}>p$, secondly we define a node $p_{-} \leq p$, and finally, we prove that $\left[p_{-}, p_{+}\right)$is maximal branching free. If for all $p^{\prime}>p$ and all $p^{\prime \prime}<p^{\prime}, p^{\prime \prime}$ and $p$ are comparable, set $p_{+}:=\infty$. Note that $\left[p, p_{+}\right)$is branching free. Otherwise, by well-foundedness and upwards linearity, there is a minimal element $p_{+}>p$ for which there exists $p^{\prime}<p_{+}$incomparable to $p$. Thus, $\left[p, p_{+}\right)$is branching free.

Lemma 2.2 implies that, for all $p_{1} \leq p, p_{2} \leq p$, if $\left[p_{1}, p_{+}\right)$and $\left[p_{2}, p_{+}\right)$are branching free, then $p_{1}$ and $p_{2}$ are comparable. By well-foundedness, there is a minimal $p_{-} \leq p$ such that $I:=\left[p_{-}, p_{+}\right)$is branching free. 
In order to prove maximality, assume that for some $p_{1} \leq p<p_{2},\left[p_{1}, p_{2}\right)$ is branching free. First we show that $p_{+} \notin\left[p_{1}, p_{2}\right)$ : If $p_{+} \in\left[p_{1}, p_{2}\right)$ then, since $\left[p_{1}, p_{2}\right)$ is branching free, for every $p^{\prime}<p_{+}, p^{\prime}$ and $p_{1}$ are comparable. By upwards linearity, if $p^{\prime}$ and $p_{1}$ are comparable then also $p^{\prime}$ and $p$ are comparable. Hence, every $p^{\prime}<p_{+}$is comparable to $p$. But this contradicts the choice of $p_{+}$. Hence, we have $p_{+} \notin\left[p_{1}, p_{2}\right)$.

Since $p<p_{2}$ and $p<p_{+}, p_{2}$ and $p_{+}$are comparable. We cannot have $p_{+}<p_{2}$. Hence, $\max \left(p_{2}, p_{+}\right)=p_{+}$. By Lemma 2.3, the interval

$$
\left[\min \left(p_{1}, p_{-}\right), p_{+}\right)=\left[\min \left(p_{1}, p_{-}\right), \max \left(p_{2}, p_{+}\right)\right)
$$

is branching free. But by definition of $p_{-}, I$ is the minimal branching free interval of the form $\left[p, p_{+}\right)$. Thus, $p_{-} \leq p_{1}$ and $\left[p_{1}, p_{2}\right)$ is contained in $I$. This shows that $I$ is maximal branching free.

Lemma 2.3 and 2.4 imply that every wulpo is partitioned into its maximal branching free intervals.

Lemma 2.5. Let $\mathfrak{P}$ be a wulpo and $I \neq J$ two different maximal branching free intervals of $\mathfrak{P}$. For $p_{1} \in I$ and $p_{2} \in J$, if $p_{1}<p_{2}$ then there is a $p \in \mathfrak{P}$ such that $p<p_{2}$ but $p$ and $p_{1}$ are incomparable, i.e., $\downarrow p_{2} \neq \downarrow p_{1} \cup\left[p_{1}, p_{2}\right)$.

Proof. Heading for a contradiction, assume that

$$
\downarrow p_{2}=\downarrow p_{1} \cup\left[p_{1}, p_{2}\right) .
$$

Note that there is a $p_{m} \in \mathfrak{P} \cup\{\infty\}$ such that $K:=\left[p_{1}, p_{m}\right)=\left\{p \in \mathfrak{P} \mid p_{1} \leq p \leq p_{2}\right\}$. Due to (2.1), $K$ is branching free. Using Lemma 2.3 we conclude that $I \cup K \cup J$ is branching free contradicting maximality of $I$ and $J$.

We finally discuss the replacement of maximal branching free intervals in wulpo's. Let $\mathfrak{P}=(P, \leq)$ be a wulpo and $I=\left[p_{0}, p_{1}\right)$ a maximal branching free interval. For each $i \in I$ and each $p \in P \backslash I$ we have

$$
\begin{aligned}
& p \leq i \text { if and only if } p \leq i^{\prime} \text { for all } i^{\prime} \in I \text { and } \\
& p \geq i \text { if and only if } p \geq i^{\prime} \text { for all } i^{\prime} \in I
\end{aligned}
$$

Let $J$ be some ordinal. Then we can replace $I$ by $J$ and obtain the wulpo $\mathfrak{P}^{\prime}=\left((P \backslash I) \sqcup J, \leq^{\prime}\right)$ where $p \leq^{\prime} p^{\prime}$ if

(1) $p, p^{\prime} \in P \backslash I$ and $p \leq p^{\prime}$,

(2) $p, p^{\prime} \in J$ and $p \leq p^{\prime}$ in $J$,

(3) $p \in P \backslash I, p^{\prime} \in J$ and $p \leq p_{0}$, or

(4) $p \in J, p^{\prime} \in P \backslash I$ and $p_{0} \leq p^{\prime}$.

Note that the maximal branching free intervals of $\mathfrak{P}^{\prime}$ are $J$ and the maximal branching free intervals of $\mathfrak{P}$ except for $I$. Analogously, one can also replace simultaneously all maximal branching free intervals of a wulpo by new ordinals. This kind of replacement will be used in Lemma 3.15. 
2.2. Ordinal arithmetic and the ordinal rank. We use standard terminology concerning ordinals; see e.g. [25]. We briefly recall the operations of natural sum and natural product on ordinals. The natural sum of ordinals $\alpha$ and $\beta$, denoted by $\alpha \oplus \beta$, is the largest (well-founded) linearization of $\alpha \sqcup \beta$ (cf. [7, where the following statements can be found). It is commutative, associative and strictly monotone, i.e., $\alpha \oplus \beta<\alpha \oplus \gamma$ if and only if $\beta<\gamma$. Furthermore,

$$
\alpha \oplus \beta=\omega^{\gamma} \text { implies } \alpha=\omega^{\gamma} \text { or } \beta=\omega^{\gamma} \text {. }
$$

The natural product (cf. 7]) of $\alpha$ and $\beta$, denoted as $\alpha \otimes \beta$, is the largest (well-founded) linearization of the direct (Cartesian) product of $\alpha$ and $\beta$ with the componentwise order. It is commutative, associative, and strictly monotone, i.e., for $\alpha \neq 0$ we have $\alpha \otimes \beta<\alpha \otimes \gamma$ if and only if $\beta<\gamma$. Furthermore,

$$
\alpha \otimes \beta=\omega^{\omega^{\gamma}} \text { implies } \alpha=\omega^{\omega^{\gamma}} \text { or } \beta=\omega^{\omega^{\gamma}} .
$$

Lemma 2.6. Let $\alpha, \beta, \gamma$ be ordinals. Then

$$
(\alpha \otimes \beta)+(\alpha \otimes \gamma) \leq \alpha \otimes(\beta+\gamma)
$$

where + is the usual sum of ordinals.

Proof. Observe that $(\alpha \otimes \beta)+(\alpha \otimes \gamma)$ is a well-order with domain $(\alpha \times \beta) \sqcup(\alpha \times \gamma)=\alpha \times(\beta \sqcup \gamma)$ such that for all $a<a^{\prime} \in \alpha, b<b^{\prime} \in \beta$ and $c<c^{\prime} \in \gamma$ we have $(a, b)<\left(a^{\prime}, c\right),(a, b)<\left(a^{\prime}, b\right)$, $(a, c)<\left(a^{\prime}, c\right),(a, b)<\left(a, b^{\prime}\right)$, and $(a, c)<\left(a, c^{\prime}\right)$. Thus, we conclude that it is a linearization of the product of the orders $\alpha$ and $\beta+\gamma$. Since $\alpha \otimes(\beta+\gamma)$ is the largest linearization of these two orders, the claim follows immediately.

Let us recall the (ordinal) rank of a well-founded partial order. For a set of ordinals $M$, we denote by $\sup (M)$ the supremum of $M$, where $\sup (\emptyset)=0$.

Definition 2.7. Let $\mathfrak{P}$ be a well-founded partial order. The rank of an element $p \in \mathfrak{P}$ is inductively defined by

$$
\operatorname{rank}(p, \mathfrak{P})=\sup \left\{\operatorname{rank}\left(p^{\prime}, \mathfrak{P}\right)+1 \mid p^{\prime}<p \in P\right\} .
$$

The rank of $\mathfrak{P}$ (also called the ordinal height of $\mathfrak{P}$ ) is

$$
\operatorname{rank}(\mathfrak{P})=\sup \{\operatorname{rank}(p, \mathfrak{P})+1 \mid p \in P\} .
$$

If the partial order $\mathfrak{P}$ is clear from the context, we will write rank $(p)$ instead of $\operatorname{rank}(p, \mathfrak{P})$. Note that $\operatorname{rank}(p, \mathfrak{P})=\operatorname{rank}\left(\left.\mathfrak{P}\right|_{\downarrow p}\right)$. Also note that the rank of the ordinal $\alpha$ is exactly $\alpha$. Next, we collect some useful facts about the rank.

Lemma 2.8. Let $\mathfrak{P}=(P, \leq)$ be a well-founded partial order and $S \subseteq P$ a subset. The induced suborder $\left.\mathfrak{P}\right|_{S}$ has rank at most $\operatorname{rank}(\mathfrak{P})$.

Proof. By induction on the rank of an element $s \in S$ (with respect to $\mathfrak{P}$ ) one easily shows that the rank of $s$ in $\mathfrak{P} \uparrow_{S}$ is bounded by the rank of $s$ in $\mathfrak{P}$. 
We will also use a result proved by Khoussainov and Minnes on decomposition of partial orders into suborders.

Lemma 2.9 ([16], Lemma 3.3). Let $\mathfrak{P}=(P, \leq)$ be a well-founded partial order, and let $P=P_{1} \sqcup P_{2}$ be a partition of the domain of $\mathfrak{P}$. Setting $\mathfrak{P}_{i}=\mathfrak{P} \uparrow_{P_{i}}$ for $i \in\{1,2\}$, we have $\operatorname{rank}(\mathfrak{P}) \leq \operatorname{rank}\left(\mathfrak{P}_{1}\right) \oplus \operatorname{rank}\left(\mathfrak{P}_{2}\right)$.

Lemma 2.10. Let $\mathfrak{P}=(P, \leq)$ be a well-founded partial order and $p_{1} \in P, p_{2} \in P \cup\{\infty\}$ with $p_{1} \leq p_{2}$. If $\left[p_{1}, p_{2}\right)$ is branching free, then $\operatorname{rank}(p)=\operatorname{rank}\left(p_{1}\right)+\operatorname{rank}\left(\left[p_{1}, p\right)\right)$ for each $p \in\left[p_{1}, p_{2}\right)$. Moreover, if $p_{2} \in P$, then $\operatorname{rank}\left(p_{2}\right) \geq \operatorname{rank}\left(p_{1}\right)+\operatorname{rank}\left(\left[p_{1}, p_{2}\right)\right)$.

Proof. Let $p \in\left[p_{1}, p_{2}\right)$. We prove the first statement of the lemma by induction on $\alpha:=$ $\operatorname{rank}\left(\left[p_{1}, p\right)\right)$. We distinguish three cases:

Case 1. $\alpha=0$. We must have $\left[p_{1}, p\right)=\emptyset$, i.e., $p_{1}=p_{2}$. Hence $\operatorname{rank}\left(p_{2}\right)=\operatorname{rank}\left(p_{1}\right)+0$.

Case 2. $\alpha$ is a successor ordinal, i.e., $\alpha=\beta+1$. Then by definition of the rank there is an element $p^{\prime} \in\left[p_{1}, p\right)$ such that

$$
\operatorname{rank}\left(p^{\prime},\left[p_{1}, p\right)\right)=\operatorname{rank}\left(\left[p_{1}, p^{\prime}\right)\right)=\beta
$$

Moreover, $\operatorname{rank}(p) \geq \operatorname{rank}\left(p^{\prime}\right)+1$ since $p^{\prime}<p$. By induction hypothesis, we conclude that

$$
\operatorname{rank}(p) \geq \operatorname{rank}\left(p^{\prime}\right)+1=\operatorname{rank}\left(p_{1}\right)+\beta+1=\operatorname{rank}\left(p_{1}\right)+\alpha .
$$

Moreover, for every $p^{\prime} \in\left[p_{1}, p\right)$ we must have $\operatorname{rank}\left(\left[p_{1}, p^{\prime}\right)\right) \leq \beta$. Since $\left[p_{1}, p_{2}\right)$ is branching free, we have $\downarrow p^{\prime}=\downarrow p_{1}+\left[p_{1}, p^{\prime}\right)$ for every $p^{\prime}<p$. Therefore

$$
\operatorname{rank}(p)=\sup \left\{\operatorname{rank}\left(p^{\prime}\right)+1 \mid p^{\prime} \in\left[p_{1}, p\right)\right\} \text {. }
$$

By induction,

$$
\operatorname{rank}\left(p^{\prime}\right)=\operatorname{rank}\left(p_{1}\right)+\operatorname{rank}\left(\left[p_{1}, p^{\prime}\right)\right) \leq \operatorname{rank}\left(p_{1}\right)+\beta .
$$

Hence, $\operatorname{rank}(p) \leq \operatorname{rank}\left(p_{1}\right)+\beta+1=\operatorname{rank}\left(p_{1}\right)+\alpha$.

Case 3. $\alpha$ is a limit ordinal. Since $\left[p_{1}, p_{2}\right)$ is branching free, for each $p^{\prime}<p$ one of the following holds.

(1) $p^{\prime} \in\left[p_{1}, p\right)$ and there is some $\beta<\alpha$ such that $\operatorname{rank}\left(\left[p_{1}, p^{\prime}\right)\right)=\beta$. By induction hypothesis, $\operatorname{rank}\left(p^{\prime}\right)=\operatorname{rank}\left(p_{1}\right)+\beta$.

(2) $p^{\prime}<p_{1}$ whence $\operatorname{rank}\left(p^{\prime}\right) \leq \operatorname{rank}\left(p_{1}\right)$.

Thus, we have

$$
\begin{aligned}
\operatorname{rank}(p) & \leq \sup \left\{\operatorname{rank}\left(p_{1}\right)+\beta+1 \mid \beta<\alpha\right\} \\
& =\sup \left\{\operatorname{rank}\left(p_{1}\right)+\beta \mid \beta<\alpha\right\} \\
& =\operatorname{rank}\left(p_{1}\right)+\alpha .
\end{aligned}
$$

On the other hand, $\operatorname{rank}\left(\left[p_{1}, p\right)\right)=\alpha$ implies that for each $\beta<\alpha$ there is a node $p_{\beta} \in\left[p_{1}, p\right)$ such that $\beta \leq \operatorname{rank}\left(\left[p_{1}, p_{\beta}\right)<\alpha\right.$. By induction, we get $\operatorname{rank}\left(p_{\beta}\right) \geq \operatorname{rank}\left(p_{1}\right)+\beta$. Thus,

$$
\begin{aligned}
\operatorname{rank}(p) & \geq \sup \left\{\operatorname{rank}\left(p_{\beta}\right)+1 \mid \beta<\alpha\right\} \\
& \geq \sup \left\{\operatorname{rank}\left(p_{1}\right)+\beta+1 \mid \beta<\alpha\right\} \\
& =\sup \left\{\operatorname{rank}\left(p_{1}\right)+\beta \mid \beta<\alpha\right\} \\
& =\operatorname{rank}\left(p_{1}\right)+\alpha .
\end{aligned}
$$


Finally, the second statement of the lemma follows easily:

$$
\begin{aligned}
\operatorname{rank}\left(p_{2}\right) & \geq \sup \left\{\operatorname{rank}(p)+1 \mid p \in\left[p_{1}, p_{2}\right)\right\} \\
& =\sup \left\{\operatorname{rank}\left(p_{1}\right)+\operatorname{rank}\left(\left[p_{1}, p\right)\right)+1 \mid p \in\left[p_{1}, p_{2}\right)\right\} \\
& =\operatorname{rank}\left(p_{1}\right)+\operatorname{rank}\left(\left[p_{1}, p_{2}\right)\right)
\end{aligned}
$$

This concludes the proof of the lemma.

Corollary 2.11. Let $\mathfrak{P}$ be a partial order and $p_{1} \leq p_{2} \in \mathfrak{P}$ such that $\left[p_{1}, p_{2}\right)$ is branching free. For every $p \in\left[p_{1}, p_{2}\right), \operatorname{rank}(p)<\operatorname{rank}\left(p_{1}\right)+\operatorname{rank}\left(\left[p_{1}, p_{2}\right)\right)$.

2.3. Finitely labeled trees. We identify a non-empty, finite, prefix-closed subset $T \subseteq$ $\{0,1\}^{*}$ with the tree $(T, \succeq)$ and call $T$ a finite binary tree. Note that $\varepsilon$ is the largest element of the inverse prefix relation $\succeq$ and hence the root of the tree. We denote the set of all finite binary trees as $\mathcal{T}_{2}^{\text {fin }}$. For $T \in \mathcal{T}_{2}^{\text {fin }}$ let

$$
\operatorname{fr}(T)=T\{0,1\} \backslash T \text { and } \operatorname{cl}(T)=T \cup \operatorname{fr}(T)
$$

be the frontier and closure of $T$. Notice that $\mathrm{cl}(T) \in \mathcal{T}_{2}^{\text {fin }}$.

A finite $\Sigma$-labeled binary tree is a pair $(T, \lambda)$, where $T \in \mathcal{T}_{2}^{\text {fin }}$ and $\lambda: T \rightarrow \Sigma$ is a labeling function. We denote the set of all finite $\Sigma$-labeled binary trees by $\mathcal{T}_{2, \Sigma}^{\text {fin }}$; elements of $\mathcal{T}_{2, \Sigma}^{\text {fin }}$ will be denoted by lower case letters $(s, t, \ldots)$. When $\Sigma$ is the singleton set $\{\sharp\}$, we will simply consider a tree $t \in \mathcal{T}_{2, \Sigma}^{\text {fin }}$ as unlabeled, i.e., $t \in \mathcal{T}_{2}^{\text {fin }}$. The set of leaves of $t=(T, \lambda)$ is leaves $(t)=$ leaves $(T, \succeq)$. We use the following operations on labeled trees $t=(T, \lambda) \in \mathcal{T}_{2, \Sigma}^{\text {fin }}$. For $d \in T$, the subtree rooted at $d$ is the tree $t(d)=(U, \mu) \in \mathcal{T}_{2, \Sigma}^{\text {fin }}$ with $U=\left\{u \in\{0,1\}^{*} \mid d u \in T\right\}$ and $\mu(u)=\lambda(d u)$. For trees $t_{1}, \ldots, t_{n} \in \mathcal{T}_{2, \Sigma}^{\text {fin }}$ and nodes $d_{1}, \ldots, d_{n} \in \mathrm{cl}(T)$ forming an antichain (i.e., $d_{i}$ is not a prefix of $d_{j}$ for $i \neq j$ ) we consider the tree $t\left[d_{1} / t_{1}, \ldots, d_{n} / t_{n}\right] \in \mathcal{T}_{2, \Sigma}^{\text {fin }}$ which is obtained from $t$ by simultaneously replacing for each $i$ the subtree rooted at $d_{i}$ by $t_{i}$. Formally, $t\left[d_{1} / t_{1}, \ldots, d_{n} / t_{n}\right]=(V, \nu)$ is defined by

$$
\begin{gathered}
V=\left(T \backslash\left(\left\{d_{1}, \ldots, d_{n}\right\}\{0,1\}^{*}\right)\right) \cup \bigcup_{1 \leq i \leq n} d_{i} T_{i} \text { and } \\
\nu(v)= \begin{cases}\lambda_{i}(u) & \text { if } v=d_{i} u \text { for some (unique) } i \in\{1, \ldots, n\}, \\
\lambda(v) & \text { otherwise, }\end{cases}
\end{gathered}
$$

where $t_{i}=\left(T_{i}, \lambda_{i}\right)$.

We fix a new symbol $\diamond \notin \Sigma$. Let $\Sigma_{\diamond}$ denote the set $\Sigma \sqcup\{\diamond\}$ and $\Sigma_{\diamond}^{n}$ the $n$-fold product $\Sigma_{\diamond} \times \cdots \times \Sigma_{\diamond}$. Let $\bar{t}=\left(t_{1}, \ldots, t_{n}\right) \in\left(\mathcal{T}_{2, \Sigma}^{\text {fin }}\right)^{n}$ be a tuple of trees with $t_{i}=\left(T_{i}, \lambda_{i}\right)$. The convolution of $\bar{t}$ is the $\Sigma_{\diamond}^{n}$-labeled binary tree $\otimes \bar{t}=(T, \lambda) \in \mathcal{T}_{2, \Sigma_{\diamond}^{n}}^{\text {fin }}$ defined by

$$
\begin{aligned}
T & =\bigcup_{1 \leq i \leq n} T_{i} \text { and } \\
\lambda(u) & =\left(\lambda_{1}^{\prime}(u), \ldots, \lambda_{n}^{\prime}(u)\right), \text { where } \\
\lambda_{i}^{\prime}(u) & = \begin{cases}\lambda_{i}(u) & \text { if } u \in T_{i}, \\
\diamond & \text { if } u \in T \backslash T_{i} .\end{cases}
\end{aligned}
$$


Instead of $\otimes\left(t_{1}, t_{2}, \ldots, t_{n}\right)$ we sometimes also write $t_{1} \otimes \cdots \otimes t_{n}$. Finally, for any $n$-ary relation $R \subseteq\left(\mathcal{T}_{2, \Sigma}^{\text {fin }}\right)^{n}$ we define

$$
\otimes R=\{\otimes \bar{t} \mid \bar{t} \in R\} \subseteq \mathcal{T}_{2, \Sigma_{\diamond}^{n}}^{\text {fin }}
$$

2.4. Tree automata and tree-automatic structures. Let $\Sigma$ be a finite alphabet. A (top-down) tree automaton over $\Sigma$ is a tuple $\mathcal{A}=(Q, \Delta, I, F)$, where $Q$ is the finite set of states, $I \subseteq Q$ is the set of initial states, $F \subseteq Q$ is the set of final states, and $\Delta \subseteq(Q \backslash F) \times \Sigma \times Q \times Q$ is the transition relation 1$]$ Given a finite $\Sigma$-labeled binary tree $t=(T, \lambda) \in \mathcal{T}_{2, \Sigma}^{\text {fin }}$, a successful run of $\mathcal{A}$ on $t$ is a mapping $\rho: \operatorname{cl}(T) \rightarrow Q$ such that

(1) $\rho(\varepsilon) \in I$,

(2) $\rho(\operatorname{fr}(T)) \subseteq F$, and

(3) $(\rho(d), \lambda(\bar{d}), \rho(d 0), \rho(d 1)) \in \Delta$ for every $d \in T$.

Let $L(\mathcal{A})$ be the set of all $t \in \mathcal{T}_{2, \Sigma}^{\text {fin }}$ which admit a successful run of $\mathcal{A}$. A set $L \subseteq \mathcal{T}_{2, \Sigma}^{\text {fin }}$ is called regular if there is a tree automaton $\mathcal{A}$ over $\Sigma$ with $L=L(\mathcal{A})$.

A successful run $\rho$ of $\mathcal{A}$ on $t=(T, \lambda) \in \mathcal{T}_{2, \Sigma}^{\text {fin }}$ naturally defines a $((\Sigma \times(Q \backslash F)) \cup F)$ labeled binary tree tree $(\rho)=(\operatorname{cl}(T), \mu)$ such that $\mu(d)=(\lambda(d), \rho(d))$ for every $d \in T$ and $\mu(d)=\rho(d)$ for every $d \in \operatorname{fr}(T)$. $\operatorname{Run}(\mathcal{A}, t)$ denotes the set of all trees tree $(\rho)$ where $\rho$ is a successful run of $\mathcal{A}$ on $t$. Let $\operatorname{Run}(\mathcal{A})=\bigcup_{t \in L(\mathcal{A})} \operatorname{Run}(\mathcal{A}, t)$. This is also a regular set: a tree automaton for $\operatorname{Run}(\mathcal{A})$ can be obtained by replacing every transition $\left(p, a, p_{1}, p_{2}\right) \in \Delta$ by $\left(p,(a, p), p_{1}, p_{2}\right)$ and adding transitions $(f, f, \perp, \perp)$ for each final state $f \in F$ where $\perp$ is a new state which is the only final state of the new automaton. For notational simplicity we refer to tree $(\rho)$ simply as $\rho$.

A tree automaton $\mathcal{A}=(Q, \Delta, I, F)$ is called bottom-up deterministic if

(1) for all $p, q \in Q$ and $a \in \Sigma$ there is a unique $r \in Q$ with $(r, a, p, q) \in \Delta$ and

(2) $F$ is a singleton set.

In this situation, for $F=\left\{q_{0}\right\}$, a tree $t=(T, \lambda) \in \mathcal{T}_{2, \Sigma}^{\text {fin }}$, a node $u \in \mathrm{cl}(T)$, a set $U \subseteq \operatorname{fr}(T)$ and a map $\zeta: U \rightarrow Q$ we recursively define a state $\mathcal{A}(t, u, \zeta) \in Q$ by

$$
\mathcal{A}(t, u, \zeta)= \begin{cases}r & \text { if } u \in T \text { and }(r, \lambda(u), \mathcal{A}(t, u 0, \zeta), \mathcal{A}(t, u 1, \zeta)) \in \Delta, \\ \zeta(u) & \text { if } u \in U, \\ q_{0} & \text { if } u \in \operatorname{fr}(T) \backslash U\end{cases}
$$

We omit the second parameter if $u=\varepsilon$ and the third one if $U=\emptyset$. Notice that $\mathcal{A}(t, u)=$ $\mathcal{A}(t(u))$ for all $u \in T$. The tree $t$ admits a successful run $\rho$ of $\mathcal{A}$ if and only if $\mathcal{A}(t) \in I$. It is well known that for each tree automaton $\mathcal{A}$ one can compute a bottom-up deterministic tree automaton $\mathcal{A}^{\prime}$ with $L(\mathcal{A})=L\left(\mathcal{A}^{\prime}\right)$.

An $n$-ary relation $R \subseteq\left(\mathcal{T}_{2, \Sigma}^{\text {fin }}\right)^{n}$ is called tree-automatic if there is a tree automaton $\mathcal{A}_{R}$ over $\Sigma_{\diamond}^{n}$ such that $L\left(\mathcal{A}_{R}\right)=\otimes R$. A relational structure $\mathfrak{S}$ is called tree-automatic over $\Sigma$ if its domain $S$ is a regular subset of $\mathcal{T}_{2, \Sigma}^{\text {fin }}$ and each of its atomic relations $R^{\mathfrak{S}}$ is tree-automatic; any tuple $\mathbb{P}$ of automata that accept the domain and the relations of $\mathfrak{S}$ is called a treeautomatic presentation of $\mathfrak{S}$. In this case, we write $\mathfrak{S}(\mathbb{P})$ for $\mathfrak{S}$. If a tree-automatic structure $\mathfrak{S}$ is isomorphic to a structure $\mathfrak{S}^{\prime}$, then $\mathfrak{S}$ is called a tree-automatic copy of $\mathfrak{S}^{\prime}$ and $\mathfrak{S}^{\prime}$ is

\footnotetext{
${ }^{1}$ In contrast to the usual definition, we disallow transitions starting in final states. Obviously this restricted version of tree-automata is equivalent to the usual one. Our definition simplifies some constructions because runs on a tree $t$ only assume final states on the frontier of $t$.
} 
tree-automatically presentable. In this paper we sometimes abuse the terminology referring to $\mathfrak{S}^{\prime}$ as simply tree-automatic and calling a tree-automatic presentation of $\mathfrak{S}$ also a treeautomatic presentations of $\mathfrak{S}^{\prime}$. We also simplify our statements by saying "given/compute a tree-automatic structure $\mathfrak{S}$ " for "given/compute a tree-automatic presentation $\mathbb{P}$ of a structure $\mathfrak{S}(\mathbb{P})$ ". The structures $(\mathbb{N},+)$ and $(\mathbb{N}, \cdot)$ are examples of tree-automatic structures.

A tree-automatic structure over a singleton alphabet (i.e., the domain of the structure is a subset of $\mathcal{T}_{2}^{\text {fin }}$ ) is called unary tree-automatic. Moreover, let

$$
\mathcal{T}_{\text {bin }}=\left\{t \in \mathcal{T}_{2}^{\text {fin }} \mid \forall u \in t: u 0 \in t \Leftrightarrow u 1 \in t\right\}
$$

be the set of all finite (unlabeled) full binary trees. We will make use of the following simple lemma.

Lemma 2.12. For every tree-automatic structure $\mathfrak{S}$ there is an isomorphic unary treeautomatic structure $\mathfrak{S}^{\prime}$ whose domain is a subset of $\mathcal{T}_{\text {bin. }}$. Moreover, there is a computable isomorphism from $\mathfrak{S}$ to $\mathfrak{S}^{\prime}$.

Proof. Let $\Sigma$ be some finite alphabet; w.l.o.g. assume that $\Sigma=\{1,2, \ldots, n\}$. For $1 \leq i \leq n$ let $a_{i}=\operatorname{pref}\left(\left\{0,10,110, \ldots, 1^{i-1} 0,1^{i}\right\}\right) \in \mathcal{T}_{\text {bin }}$. We inductively define an injective mapping unlabel : $\mathcal{T}_{2, \Sigma}^{\text {fin }} \rightarrow \mathcal{T}_{\text {bin }}$ as follows: Let $t=(T, \lambda) \in \mathcal{T}_{2, \Sigma}^{\text {fin }}$ and for $i \in\{0,1\}$ let $t_{i}=t(i)$ be the subtree of $t$ rooted at node $i$, where we set $t_{i}=\emptyset$ if $i \notin T$. Then

$$
\text { unlabel }(t)=\{\varepsilon, 0\} \cup 00 \text { unlabel }\left(t_{0}\right) \cup 01 \text { unlabel }\left(t_{1}\right) \cup 1 a_{\lambda(\varepsilon)},
$$

where we set unlabel $(\emptyset)=\emptyset$. By induction over the size of $t$ it easily follows that the mapping unlabel is indeed injective. We show that for every tree-automatic relation $R \subseteq\left(\mathcal{T}_{2, \Sigma}^{\text {fin }}\right)^{k}$, the relation

$$
\text { unlabel }(R)=\left\{\left(\text { unlabel }\left(t_{1}\right), \ldots, \text { unlabel }\left(t_{k}\right)\right) \mid\left(t_{1}, \ldots, t_{k}\right) \in R\right\}
$$

is also tree-automatic. Suppose $\mathcal{A}=(Q, \Delta, I, F)$ is a tree automaton recognizing the relation $R$. We construct a (top-down) tree automaton $\mathcal{A}^{\prime}$ as follows: The state set of $\mathcal{A}^{\prime}$ contains the set

$$
Q \cup(Q \times Q) \cup\{\diamond, 1,2, \ldots, n\}^{n} \cup\{\perp\}
$$

(in addition $\mathcal{A}^{\prime}$ contains some auxiliary states that we do not specify), where $\perp$ is final (states from $F$ are no longer final). For a state $q \in Q, \mathcal{A}^{\prime}$ contains the following transitions (we omit here the unique node label, which formally should be the second component of every transition):

$$
\left(q,(p, r),\left(x_{1}, \ldots, x_{k}\right)\right) \text { if }\left(q,\left(x_{1}, \ldots, x_{k}\right), p, r\right) \in \Delta \quad \text { and }(q, \perp, \perp) \text { for } q \in F \text {. }
$$

For a state $(p, r) \in Q \times Q, \Delta^{\prime}$ contains the following transitions:

$$
((p, r), p, r) \text {. }
$$

Finally, the tree automaton $\mathcal{A}^{\prime}$ contains additional states and transitions such that from a state $\left(x_{1}, \ldots, x_{k}\right) \in\{\diamond, 1,2, \ldots, n\}^{n}$ only the tree $\operatorname{pref}\left(s_{1} \otimes s_{2} \otimes \cdots \otimes s_{n}\right)$ with

$$
s_{i}= \begin{cases}a_{x_{i}} & \text { if } x_{i} \in\{1, \ldots, n\}, \\ \{\varepsilon\} & \text { if } x_{i}=\diamond .\end{cases}
$$

is accepted. Now let $t_{1}, \ldots, t_{k} \in \mathcal{T}_{2, \Sigma}^{\text {fin }}$ and $q \in Q$. A straightforward induction on the size of trees shows that $\mathcal{A}$ accepts the convolution $t_{1} \otimes \cdots \otimes t_{k}$ via a run $\rho$ with $\rho(\varepsilon)=q$ if and only if $\mathcal{A}^{\prime}$ accepts unlabel $\left(t_{1}\right) \otimes \cdots \otimes$ unlabel $\left(t_{k}\right)$ via a run $\rho^{\prime}$ with $\rho^{\prime}(\varepsilon)=q$. 
The above considerations shows that for every tree-automatic structure $\mathfrak{S}$ there exists an isomorphic unary tree-automatic structure $\mathfrak{S}^{\prime}$, whose domain is a subset of $\mathcal{T}_{\text {bin. }}$. An isomorphism between $\mathfrak{S}$ and $\mathfrak{S}^{\prime}$ is given by the computable mapping unlabel. This proves the lemma.

Consider $\mathrm{FO}[\tau]+\exists^{\infty}+\exists^{\text {chain }}$, the first-order logic over the signature $\tau$ extended by the quantifiers $\exists^{\infty}$ ("there are infinitely many") and the chain-quantifier $\exists^{\text {chain }}$ (if $\varphi(x, y)$ is some formula, then $\exists^{\text {chain }} \varphi(x, y)$ asserts that $\varphi$ is a partial order and there is an infinite increasing $\varphi$-chain). Let $\varphi\left(x_{1}, \ldots, x_{m}, y_{1}, \ldots, y_{n}\right)$ be a formula of this logic in the signature of some structure $\mathfrak{S}$. For all $\bar{b} \in S^{n}$ we define the $m$-ary relation

$$
\varphi^{\mathfrak{S}}(\cdot, \bar{b})=\left\{\bar{a} \in S^{m} \mid \mathfrak{S} \models \varphi(\bar{a}, \bar{b})\right\}
$$

on $\mathfrak{S}$. In case of $n=0$ we simply write $\varphi^{\mathfrak{S}}$ instead of $\varphi^{\mathfrak{S}}(\cdot)$. The following theorem from [3, 13, 26] lays out the main motivation for investigating tree-automatic structures.

Theorem 2.13. From a tree-automatic presentation $\mathbb{P}$ of a structure $\mathfrak{S}(\mathbb{P})$ over the signature $\tau$ and an $\mathrm{FO}[\tau]+\exists^{\infty}+\exists^{\text {chain }}$-formula $\varphi(\bar{x})$ one can compute a tree automaton accepting $\otimes \varphi^{\mathfrak{S}(\mathbb{P})}$. In particular, the $\mathrm{FO}[\tau]+\exists^{\infty}+\exists^{\text {chain }}$-theory of any tree-automatic structure $\mathfrak{S}$ is (uniformly) decidable.

Note that the property of being a tree is expressible in $\mathrm{FO}[\leq]+\exists^{\infty}$. Moreover, the chain-quantifier allows to define well-foundedness of a tree. Hence, we obtain:

Corollary 2.14. The class of tree-automatic well-founded trees is decidable.

Let $\mathcal{K}$ be a class of tree-automatic presentations. The isomorphism problem $\operatorname{Iso}(\mathcal{K})$ is the set of pairs $\left(\mathbb{P}_{1}, \mathbb{P}_{2}\right) \in \mathcal{K} \times \mathcal{K}$ of tree-automatic presentations with $\mathfrak{S}\left(\mathbb{P}_{1}\right) \cong \mathfrak{S}\left(\mathbb{P}_{2}\right)$. If $\mathcal{K}$ is the class of tree-automatic presentations for a class $\mathcal{C}$ of relational structures (e.g. trees), then we briefly speak of the isomorphism problem for (tree-automatic members of) $\mathcal{C}$. The isomorphism problem for the class of all tree-automatic structures is complete for $\Sigma_{1}^{1}$, the first level of the analytical hierarchy; this holds already for string-automatic trees [18, 20].

\section{BOUNDING THE RANK OF TREE-AUTOMATIC WULPO'S AND WELL-FOUNDED TREES}

In this section we show that the rank of a tree-automatic wulpo is strictly below $\omega^{\omega^{\omega}}$. As a corollary, we obtain that the rank of every tree-automatic well-founded tree is strictly below $\omega^{\omega}$. The first part of our proof is a refinement of Delhommé's decomposition theorem for tree-automatic structures [9]. This result shows that for a given tree-automatic structure $\mathfrak{A}$ and a first-order formula $\varphi(x, \bar{y})$ all substructures induced by $\varphi$ (via different tuples $\bar{s}$ of parameters) are composed from a finite set $\mathcal{S}_{\varphi}^{\mathfrak{A}}$ of structures using the operations of box-augmentation and sum-augmentation. Roughly speaking, a structure $\mathfrak{A}$ is a sumaugmentation of structures $\mathfrak{B}_{1}, \ldots, \mathfrak{B}_{n}$ if there is a finite partition of the domain of $\mathfrak{A}$ which induces the substructures $\mathfrak{B}_{1}, \ldots, \mathfrak{B}_{n}$ (see Definition 3.1). The structure $\mathfrak{A}$ is a boxaugmentation of $\mathfrak{B}_{1}, \ldots, \mathfrak{B}_{n}$ if its domain is a finite product of copies of the domains of $\mathfrak{B}_{1}, \ldots, \mathfrak{B}_{n}$ such that fixing all but the $i^{\text {th }}$ component of this product arbitrarily results in a structure isomorphic to $\mathfrak{B}_{i}$ (see Definition 3.1).

Let $\nu$ be a function assigning ordinals to structures. An ordinal $\alpha$ is $\nu$-indecomposable if for all structures $\mathfrak{B}_{1}, \ldots, \mathfrak{B}_{n}$ and all sum- or box-augmentations $\mathfrak{A}$ of them, $\nu(\mathfrak{A})=\alpha$ implies $\nu\left(\mathfrak{B}_{i}\right)=\alpha$ for some $i \in\{1, \ldots, n\}$ (see Definition 3.7). Delhommé's result implies the following. The substructures of a tree-automatic structure $\mathfrak{A}$ (induced by a fixed first-order 
formula $\varphi$ ) only take finitely many $\nu$-indecomposable values. Unfortunately, Delhommé never published a proof of this result. Since our further arguments rely on this proof, we reprove his result in Sections 3.1 and 3.2. More precisely, we strengthen his result in the following way.

- All box-augmentations occurring in the decomposition of tree-automatic substructures provided by Delhommé's result are tamely colorable. Roughly speaking, $\mathfrak{A}$ is a tamely colorable box-augmentation of $\mathfrak{B}_{1}, \ldots, \mathfrak{B}_{n}$ if there are finite colorings of the $\mathfrak{B}_{i}$ and a simple rule how to completely reconstruct $\mathfrak{A}$ from the $\mathfrak{B}_{i}$ and these colorings.

- We introduce a notion of $\nu$-indecomposability for intervals of ordinals: we say $\left[\alpha_{1}, \alpha_{2}\right]$ is $\nu$-indecomposable if

(1) for all structures $\mathfrak{B}_{1}, \ldots, \mathfrak{B}_{n}$ and all sum-augmentations $\mathfrak{A}$ of them, $\nu(\mathfrak{A})=\alpha_{2}$ implies $\nu\left(\mathfrak{B}_{i}\right)=\alpha_{2}$ for some $i \in\{1, \ldots, n\}$ and

(2) for all structures $\mathfrak{B}_{1}, \ldots, \mathfrak{B}_{n}$ and all tamely colorable box-augmentations $\mathfrak{A}$ of them, $\nu(\mathfrak{A})=\alpha_{2}$ implies $\nu\left(\mathfrak{B}_{i}\right) \in\left[\alpha_{1}, \alpha_{2}\right]$ for some $i \in\{1, \ldots, n\}$.

It follows directly that for a sequence $\alpha_{0}<\alpha_{1}<\alpha_{2}<\cdots$ of ordinals such that each interval $\left[\alpha_{i}, \alpha_{i+1}\right]$ is $\nu$-indecomposable, the substructures of a tree-automatic structure $\mathfrak{A}$ (induced by a first-order formula $\varphi$ ) take only finitely many values among the $\alpha_{i}$ under $\nu$ (Proposition 3.9).

In Section 3.3. we prove that the rank of any tree-automatic wulpo is bounded by $\omega^{\omega^{\omega}}$ and in the case of a tree-automatic well-founded forest its rank is bounded by $\omega^{\omega}$. We prove this result using rank-indecomposability: we can prove that all intervals of the form $\left[\omega^{\omega^{\alpha}}, \omega^{\omega^{\alpha+1}}\right]$ are rank-indecomposable (if we restrict the domain of rank to the class of wulpo's). Thus, there are infinitely many rank-indecomposable intervals below $\omega^{\omega^{\omega}}$. A simple transfinite induction shows that for every well-founded partial order $\mathfrak{P}$ of rank $\alpha$ and for all $\beta<\alpha$, there is a node $p \in \mathfrak{P}$ such that $\mathfrak{P} \uparrow_{\left\{p^{\prime} \mid p^{\prime}<p\right\}}$ is of rank $\beta$. Thus, for a fixed tree-automatic well-founded partial order $\mathfrak{P}$, the automaton corresponding to the formula $x<y$ induces substructures of all $\operatorname{ranks} \beta<\operatorname{rank}(\mathfrak{P})$. For a tree-automatic wulpo $\mathfrak{P}$ we have seen that these substructures may only take finitely many values of the form $\omega^{\omega^{i}}$. Thus, one concludes immediately that $\operatorname{rank}(\mathfrak{P})<\omega^{\omega^{\omega}}$. The bound on the ranks of tree-automatic forests follows from an analogous proof: writing rank $k_{\mathcal{F}}$ for the restriction of rank to forests, we can prove that the interval $\left[\omega^{i}, \omega^{i+1}\right]$ is rank ${ }_{\mathcal{F}}$-indecomposable.

Note that Delhommé's original result is too weak for our proof. Using the definition of rank-indecomposable values with respect to all box-augmentations, the ordinals 0 and 1 are the only rank-indecomposable values. The problem is that any forest containing an infinite antichain is the box-augmentation of two infinite antichains. Note that an infinite antichain has rank 1 . Hence, ordinals above 1 are not rank $_{\mathcal{F}}$-indecomposable with respect to Delhommé's original definition. The crucial point is that such a box-augmentation of two infinite antichains in general is not tamely colorable.

3.1. Delhommé's decomposition theorem for tree-automatic structures. In this section we reprove Delhommé's decomposition theorem [9]. Beforehand, we give the precise definitions of sum- and box-augmentations. After providing a proof of Delhommé's original result, we introduce tamely colorable box-augmentations and improve this result. For the rest of this section, we fix a relational signature $\tau=(\mathcal{R}$, ar $)$.

Definition 3.1. Let $\mathfrak{A}$ and $\mathfrak{B}_{1}, \ldots, \mathfrak{B}_{n}$ be $\tau$-structures. 
(1) We say that $\mathfrak{A}$ is a sum-augmentation of $\left(\mathfrak{B}_{1}, \ldots, \mathfrak{B}_{n}\right)$ if there is a partition

$$
A=\bigsqcup_{1 \leq i \leq n} A_{i}
$$

of the domain of $\mathfrak{A}$ such that $\mathfrak{A} \uparrow_{A_{i}} \cong \mathfrak{B}_{i}$ for all $1 \leq i \leq n$.

(2) We say that $\mathfrak{A}$ is a box-augmentation of $\left(\mathfrak{B}_{1}, \ldots, \mathfrak{B}_{n}\right)$ if there is a bijection

$$
\eta: \prod_{1 \leq i \leq n} B_{i} \rightarrow A
$$

such that for each $1 \leq k \leq n$ and all $\bar{d}=\left(d_{1}, \ldots, d_{k-1}, d_{k+1}, \ldots, d_{n}\right) \in \prod_{1 \leq i \leq n, i \neq k} B_{i}$

$$
\begin{aligned}
& \eta_{k}^{\bar{d}}: B_{k} \rightarrow A \\
& \eta_{k}^{\bar{d}}(e)=\eta\left(d_{1}, \ldots d_{k-1}, e, d_{k+1}, \ldots d_{n}\right)
\end{aligned}
$$

is an (isomorphic) embedding of $\mathfrak{B}_{k}$ into $\mathfrak{A}$.

Remark 3.2. Whenever $\mathfrak{A}$ is a sum- or box-augmentation of $\left(\mathfrak{B}_{1}, \ldots, \mathfrak{B}_{n}\right)$, the $\mathfrak{B}_{i}$ are substructures of $\mathfrak{A}$. In particular, if $\mathfrak{A}$ is a forest or a wulpo, the $\mathfrak{B}_{i}$ are also forests or wulpo's, respectively.

To simplify notation, for a $\tau$-structure $\mathfrak{A}$, an $\mathrm{FO}[\tau]+\exists^{\infty}+\exists^{\text {chain }}$-formula $\varphi\left(x, y_{1}, \ldots, y_{n}\right)$, and a tuple $\bar{s} \in A^{n}$ we use $\mathfrak{A} \uparrow_{\varphi, \bar{s}}$ as an abbreviation for $\mathfrak{A} \uparrow_{\varphi^{\mathfrak{A}}(\cdot, \bar{s})}$. Delhommé's decomposition theorem is the following.

Theorem 3.3. From a given tree-automatic $\tau$-structure $\mathfrak{A}$ and an $\mathrm{FO}[\tau]+\exists^{\infty}+\exists^{\text {chain }}$ formula $\varphi\left(x, y_{1}, \ldots, y_{n}\right)$, one can compute a finite set $\mathcal{S}_{\varphi}^{\mathfrak{A}}$ of tree-automatic structures such that for all $\bar{s} \in A^{n}$ the substructure $\mathfrak{A}_{\varphi, \bar{s}}$ is a sum-augmentation of box-augmentations of elements from $\mathcal{S}_{\varphi}^{\mathfrak{A}}$.

Proof. Let $\mathcal{A}_{R}$, where $R \in \mathcal{R}$, (resp. $\mathcal{A}_{\varphi}$ ) be a bottom-up deterministic tree automaton accepting $\otimes R^{\mathfrak{A}}\left(\right.$ resp. $\left.\otimes \varphi^{\mathfrak{A}}\right)$ and $Q_{R}$ (resp. $\left.Q_{\varphi}\right)$ be its state set. For each $t=(T, \lambda) \in \mathcal{T}_{2, \Sigma}^{\text {fin }}$ and all $R \in \mathcal{R}$ of arity $r$ we define $\underset{R}{\otimes} t=\otimes(t, \ldots, t) \in \mathcal{T}_{2, \Sigma_{\diamond}^{r}}^{\text {fin }}$ where the convolution is made up of $r$ many copies of $t$. We further consider the tree $t \underset{n}{\otimes} \emptyset=\left(T, \lambda^{\prime}\right) \in \mathcal{T}_{2, \Sigma_{\diamond}^{1+n}}^{\text {fin }}$ with $\lambda^{\prime}(u)=(\lambda(u), \diamond, \ldots, \diamond)$, where the number of $\diamond$-symbols is $n$.

As a first step towards proving the claim, we construct the set $\mathcal{S}_{\varphi}^{\mathfrak{A}}$. Before we construct the set, let us give some intuition on the structures we are interested in. $\mathcal{S}_{\varphi}^{\mathfrak{A}}$ consists of structures $\mathfrak{B}_{\gamma}$ that are substructures of the structures $\mathfrak{A} \uparrow_{\varphi, \bar{s}}$ which are obtained as follows: we fix an element $t$ of $\mathfrak{A} \uparrow_{\varphi, \bar{s}}$ and we fix a node $d$ in $t$ that is outside of the domain of $\bar{s}$. Now the domain of $\mathfrak{B}_{\gamma}$ consists of all elements of $\mathfrak{A} \uparrow_{\varphi, \bar{s}}$ that are obtained from $t$ by replacing the subtree rooted at $d$ by some other tree. Thus, the structures $\mathfrak{B}_{\gamma}$ are the substructures of all $\mathfrak{A} \uparrow_{\varphi, \bar{s}}$ whose domain is obtained by only local changes to a fixed tree.

Let $\Gamma=\prod_{R \in \mathcal{R}} Q_{R} \times Q_{\varphi} \times \prod_{R \in \mathcal{R}} 2^{Q_{R}}$. For each $\gamma=\left(\left(q_{R}\right)_{R \in \mathcal{R}}, q_{\varphi},\left(P_{R}\right)_{R \in \mathcal{R}}\right) \in \Gamma$ we define a structure $\mathfrak{B}_{\gamma}$ with domain

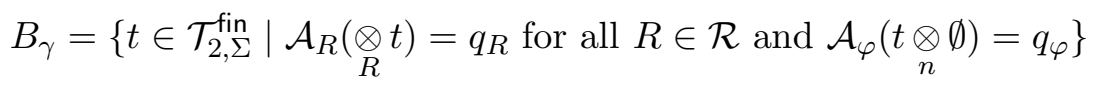

and relation $R \in \mathcal{R}$ (with arity $r=\operatorname{ar}(R)$ ) interpreted by

$$
R^{\mathfrak{B}_{\gamma}}=\left\{\bar{t} \in\left(B_{\gamma}\right)^{r} \mid \mathcal{A}_{R}(\otimes \bar{t}) \in P_{R}\right\} .
$$




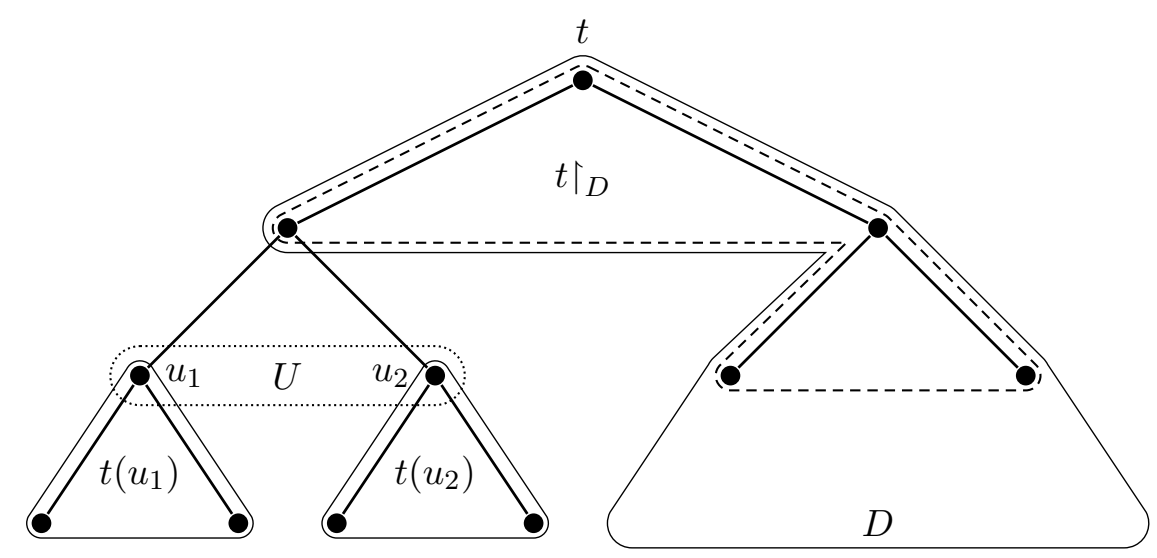

Figure 1: The $\bar{s}$-type of some example tree $t$. The dashed line surrounds $t \uparrow_{D}$ and $U$ consists of all nodes within the dotted line.

Clearly, $\mathfrak{B}_{\gamma}$ is tree-automatic. Finally, we let $\mathcal{S}_{\varphi}^{\mathfrak{A}}$ be the finite set

$$
\mathcal{S}_{\varphi}^{\mathfrak{A}}=\left\{\mathfrak{B}_{\gamma} \mid \gamma \in \Gamma\right\} .
$$

It remains to show that for each tuple $\bar{s}=\left(s_{1}, \ldots, s_{n}\right) \in \mathfrak{A}^{n}$ the substructure $\mathfrak{A} \uparrow_{\varphi, \bar{s}}$ is a sum-augmentation of box-augmentations of structures from $\left\{\mathfrak{B}_{\gamma} \mid \gamma \in \Gamma\right\}$.

For this purpose, we fix such a tuple $\bar{s}$ and consider the finite binary tree $D=\bigcup_{1 \leq i \leq n} S_{i}$, where $s_{i}=\left(S_{i}, \mu_{i}\right)$. The $\bar{s}$-type of a tree $t=(T, \lambda) \in \mathcal{T}_{2, \Sigma}^{\text {fin }}$ is the tuple

$$
\operatorname{tp}_{\bar{s}}(t)=\left(t \Gamma_{D}, U,\left(\zeta_{R}\right)_{R \in \mathcal{R}}, \zeta_{\varphi}\right),
$$

where

(1) $t \uparrow_{D}=\left(T \cap D, \lambda \uparrow_{(T \cap D)}\right) \in \mathcal{T}_{2, \Sigma}^{\text {fin }}$ is the restriction of $t$ to the domain $T \cap D$,

(2) $U=T \cap \operatorname{fr}(D) \subseteq \mathrm{fr}(T \cap D)$,

(3) $\zeta_{R}: U \rightarrow Q_{R}$ is a map with $\zeta_{R}(u)=\mathcal{A}_{R}(\underset{R}{\otimes} t(u))$, and

(4) $\zeta_{\varphi}: U \rightarrow Q_{\varphi}$ is a map with $\zeta_{\varphi}(u)=\mathcal{A}_{\varphi}(t(u) \underset{n}{\otimes} \emptyset)$.

Figure 1 illustrates the choice of $t \Gamma_{D}$ and $U$. Observe that for $t_{u}:=t(u) \underset{n}{\otimes}$ the equation

$$
\otimes(t, \bar{s})=\otimes\left(t \Gamma_{D}, \bar{s}\right)\left[\left(u / t_{u}\right)_{u \in U}\right]
$$

holds, whence

$$
\mathcal{A}_{\varphi}(\otimes(t, \bar{s}))=\mathcal{A}_{\varphi}\left(\otimes\left(t\left\lceil_{D}, \bar{s}\right), \zeta_{\varphi}\right) .\right.
$$

Therefore, $\operatorname{tp}_{\bar{s}}(t)$ determines whether $t \in \varphi^{\mathfrak{A}}(\cdot, \bar{s})$. Since $D$ and $\mathcal{R}$ are finite, there are only finitely many distinct $\bar{s}$-types. Consequently, the equivalence relation $\sim_{\bar{s}}$ on $\mathcal{T}_{2, \Sigma}^{\text {fin }}$ defined by $t \sim_{\bar{s}} t^{\prime}$ if and only if $\operatorname{tp}_{\bar{s}}(t)=\operatorname{tp}_{\bar{s}}\left(t^{\prime}\right)$ has finite index. Due to (3.3) $), \sim_{\bar{s}}$ saturates the set $\varphi^{\mathfrak{A}}(\cdot, \bar{s})$, i.e., $\varphi^{\mathfrak{A}}(\cdot, \bar{s})$ is a disjoint union of some $\sim_{\bar{s}^{-}}$-classes. Assume that $Z_{1}, \ldots, Z_{\ell} \subseteq$ $\varphi^{\mathfrak{A}}(\cdot, \bar{s})$ are these $\sim_{\bar{s}}$-classes. Then $\mathfrak{A} \uparrow_{\varphi, \bar{s}}$ is a sum-augmentation of $\left.\mathfrak{A}\right|_{Z_{1}}, \ldots, \mathfrak{A} \uparrow_{Z_{\ell}}$.

As the next step, we fix a single $\sim_{\bar{s}}$-class $Z \subseteq \varphi^{\mathfrak{A}}(\cdot, \bar{s})$. We show that $\mathfrak{Z}=\mathfrak{A} \uparrow_{Z}$ is a box-augmentation of a tuple of structures from $\mathcal{S}_{\varphi}^{\mathfrak{A}}$. Let $\left(t, U,\left(\zeta_{R}\right)_{R \in \mathcal{R}}, \zeta_{\varphi}\right)$ be the $\bar{s}$-type corresponding to $Z$. Note that the domain of $t$ is contained in the set $D$. For $u \in U$ we define

$$
\gamma(u)=\left(\left(\zeta_{R}(u)\right)_{R \in \mathcal{R}}, \zeta_{\varphi}(u),\left(P_{R}(u)\right)_{R \in \mathcal{R}}\right) \in \Gamma,
$$


where for each relation $R$

$$
P_{R}(u)=\left\{q \in Q_{R} \mid \mathcal{A}_{R}\left(\underset{R}{\otimes} t, \zeta_{R}[u \mapsto q]\right) \in I_{R}\right\} .
$$

Here $I_{R} \subseteq Q_{R}$ is the set of initial states of $\mathcal{A}_{R}$, and $\zeta_{R}[u \mapsto q]$ is the map $\zeta_{R}$ with the value at position $u$ changed to $q$. Finally, we put $\mathfrak{X}_{u}=\mathfrak{B}_{\gamma(u)}$ and denote the domain of $\mathfrak{X}_{u}$ by $X_{u}$. By definition of the the domain $B_{\gamma(u)}$ of $\mathfrak{X}_{u}$ (see (3.1) ), $y \in X_{u}$ is equivalent to

$$
\begin{aligned}
\mathcal{A}_{R}(\underset{R}{\otimes} y) & =\zeta_{R}(u) \text { for all } R \in \mathcal{R} \\
\mathcal{A}_{\varphi}(y \underset{n}{\otimes} \emptyset) & =\zeta_{\varphi}(u) .
\end{aligned}
$$

It remains to prove that $\mathfrak{Z}$ is a box-augmentation of $\left(\mathfrak{X}_{u}\right)_{u \in U}$. First, observe that the map

$$
\eta: \prod_{u \in U} X_{u} \rightarrow \mathcal{T}_{2, \Sigma}^{\text {fin }}
$$

defined by

$$
\eta\left(\left(x_{u}\right)_{u \in U}\right)=t\left[\left(u / x_{u}\right)_{u \in U}\right]
$$

is injective. Moreover, a tree $x=(X, \lambda) \in \mathcal{T}_{2, \Sigma}^{\text {fin }}$ is contained in the image of $\eta$ if and only if (1) $x \uparrow_{D}=t$,

(2) $X \cap \operatorname{fr}(D)=U$, and

(3) $x(u) \in X_{u}$ for each $u \in U$.

Note that by (3.5) and (3.6), condition (3) is equivalent to $\mathcal{A}_{R}\left(\otimes_{R} x(u)\right)=\zeta_{R}(u)$ for all $u \in U$ and $R \in \mathcal{R}$ and $\mathcal{A}_{\varphi}(x(u) \underset{n}{\otimes} \emptyset)=\zeta_{\varphi}(u)$ for all $u \in U$. Hence, the conjunction of (1), (2), and (3) is equivalent to $\operatorname{tp}_{\bar{s}}(x)=\left(t, U,\left(\zeta_{R}\right)_{R \in \mathcal{R}}, \zeta_{\varphi}\right)$ which again is equivalent to $x \in Z$. Thus, $\eta$ is a bijection onto its image $Z$.

Finally, we show that $\eta$ is a componentwise embedding of the $\left(\mathfrak{X}_{u}\right)_{u \in U}$ into $\mathfrak{Z}$. For $u_{0} \in U$ and $\bar{x}=\left(x_{u}\right)_{u \in U \backslash\left\{u_{0}\right\}} \in \prod_{u \in U \backslash\left\{u_{0}\right\}} X_{u}$ define the map $\eta_{u_{0}}^{\bar{x}}: X_{u_{0}} \rightarrow Z$ by

$$
\eta_{u_{0}}^{\bar{x}}\left(x_{u_{0}}\right)=\eta\left(\left(x_{u}\right)_{u \in U}\right)
$$

For all $R \in \mathcal{R}$ of arity $r$ and $\left(t_{1}, \ldots, t_{r}\right) \in\left(X_{u_{0}}\right)^{r}$ we have

$$
\otimes\left(\eta_{u_{0}}^{\bar{x}}\left(t_{1}\right), \ldots, \eta_{u_{0}}^{\bar{x}}\left(t_{r}\right)\right)=\left(\underset{R}{\otimes} t\left[\left(u / x_{u}\right)_{u \in U \backslash\left\{u_{0}\right\}}\right]\right)\left[u_{0} / \otimes\left(t_{1}, \ldots, t_{r}\right)\right] .
$$

Since $\mathcal{A}_{R}\left(\underset{R}{\otimes} x_{u}\right)=\zeta_{R}(u)$ for all $u \in U \backslash\left\{u_{0}\right\}$, this implies

$$
\mathcal{A}_{R}\left(\otimes\left(\eta_{u_{0}}^{\bar{x}}\left(t_{1}\right), \ldots, \eta_{u_{0}}^{\bar{x}}\left(t_{r}\right)\right)\right)=\mathcal{A}_{R}\left(\underset{R}{\otimes} t, \zeta_{R}\left[u_{0} \mapsto \mathcal{A}_{R}\left(\otimes\left(t_{1}, \ldots, t_{r}\right)\right)\right]\right) .
$$

We obtain the following chain of equivalences

$$
\begin{aligned}
\left(\eta_{u_{0}}^{\bar{x}}\left(t_{1}\right), \ldots, \eta_{u_{0}}^{\bar{x}}\left(t_{r}\right)\right) \in R^{\mathfrak{Z}} & \Longleftrightarrow\left(\eta_{u_{0}}^{\bar{x}}\left(t_{1}\right), \ldots, \eta_{u_{0}}^{\bar{x}}\left(t_{r}\right)\right) \in R^{\mathfrak{A}} \\
& \Longleftrightarrow \mathcal{A}_{R}\left(\otimes\left(\eta_{u_{0}}^{\bar{x}}\left(t_{1}\right), \ldots, \eta_{u_{0}}^{\bar{x}}\left(t_{r}\right)\right)\right) \in I_{R} \\
& \stackrel{(3.7)}{\Longleftrightarrow} \mathcal{A}_{R}\left(\otimes_{R} t, \zeta_{R}\left[u_{0} \mapsto \mathcal{A}_{R}\left(\otimes\left(t_{1}, \ldots, t_{r}\right)\right)\right]\right) \in I_{R} \\
& \stackrel{(3.4)}{\Longleftrightarrow} \mathcal{A}_{R}\left(\otimes\left(t_{1}, \ldots, t_{r}\right)\right) \in P_{R}\left(u_{0}\right) \\
& \stackrel{(3.2)}{\Longleftrightarrow}\left(t_{1}, \ldots, t_{r}\right) \in R^{\mathfrak{X}_{u_{0}}}
\end{aligned}
$$


showing that $\eta_{u_{0}}^{\bar{x}}$ is an embedding of $\mathfrak{X}_{u_{0}}$ into $\mathfrak{Z}$. Consequently, $\mathfrak{Z}$ is a box-augmentation of $\left(\mathfrak{X}_{u}\right)_{u \in U}$.

After all, let us take a closer look at the properties of the box-augmentation $\mathfrak{Z}$ of $\left(\mathfrak{X}_{u}\right)_{u \in U}$ in the previous proof. Consider some $R \in \mathcal{R}$ and let $r=\operatorname{ar}(R)$. For all $\bar{x}_{1}, \ldots, \bar{x}_{r} \in \prod_{u \in U} X_{u}$ with $\bar{x}_{i}=\left(x_{i, u}\right)_{u \in U}$ we have

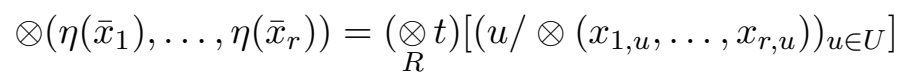

and hence

$$
\mathcal{A}_{R}\left(\otimes\left(\eta\left(\bar{x}_{1}\right), \ldots, \eta\left(\bar{x}_{r}\right)\right)\right)=\mathcal{A}_{R}(\underset{R}{\otimes} t, \zeta),
$$

where $\zeta: U \rightarrow Q_{R}$ is defined by $\zeta(u)=\mathcal{A}_{R}\left(\otimes\left(x_{1, u}, \ldots, x_{r, u}\right)\right)$. Thus, the tuple $(\zeta(u))_{u \in U}$ determines whether $\left(\eta\left(\bar{x}_{1}\right), \ldots, \eta\left(\bar{x}_{r}\right)\right) \in R^{\mathfrak{A}}$.

This observation leads to an improved version of Theorem 3.3, namely Corollary 3.6 below. As an abstraction of our observation, we introduce the notion of tamely colorable box-augmentations in the following definition. Therein for $R \in \mathcal{R}$ a finite $R$-coloring of a $\tau$-structure $\mathfrak{A}$ is a map $\sigma: A^{\operatorname{ar}(R)} \rightarrow C$ into a finite set $C$ such that for all $\bar{x}, \bar{y} \in A^{\operatorname{ar}(R)}$ with $\sigma(\bar{x})=\sigma(\bar{y})$ we have $\bar{x} \in R^{\mathfrak{A}}$ if and only if $\bar{y} \in R^{\mathfrak{A}}$.

Definition 3.4. Let $\mathcal{A}$ and $\mathfrak{B}_{1}, \ldots, \mathfrak{B}_{n}$ be $\tau$-structures. We say that $\mathfrak{A}$ is a tamely colorable box-augmentation of $\left(\mathcal{B}_{1}, \ldots, \mathfrak{B}_{n}\right)$ if

(1) there exists a bijection $\eta: \prod_{1 \leq i \leq n} B_{i} \rightarrow A$ witnessing that $\mathfrak{A}$ is a box-augmentation of $\left(\mathfrak{B}_{1}, \ldots, \mathfrak{B}_{n}\right)$ and

(2) for every $R \in \mathcal{R}$ of arity $r=\operatorname{ar}(R)$ there are finite $R$-colorings $\sigma_{i}: B_{i}^{r} \rightarrow C_{i}$ of each $\mathfrak{B}_{i}$ such that the map $\sigma: A^{r} \rightarrow C_{1} \times \cdots \times C_{n}$ mapping $\left(a_{1}, \ldots, a_{r}\right) \in A^{r}$ with $a_{j}=\eta\left(b_{j, 1}, \ldots, b_{j, n}\right)$ for $1 \leq j \leq r$ to

$$
\sigma\left(a_{1}, \ldots, a_{r}\right)=\left(\sigma_{1}\left(b_{1,1}, \ldots, b_{r, 1}\right), \ldots, \sigma_{n}\left(b_{1, n}, \ldots, b_{r, n}\right)\right),
$$

is a finite $R$-coloring of $\mathfrak{A}$.

Roughly speaking, the last condition says that the colors assigned to the tuples of the "componentwise preimages" of the $a_{i}$ under $\eta$ already determine whether $\left(a_{1}, \ldots, a_{r}\right) \in R^{\mathfrak{A}}$.

Remark 3.5. In the situation of Definition [3.4, assume that $\mathfrak{A}$ and the $\mathfrak{B}_{i}$ are directed graphs and all $C_{i}$ are the same set, say $\{1, \ldots, m\}$. For $1 \leq i \leq n$ the structure $\mathfrak{X}_{i}=$ $\left(B_{i} ; R_{1}^{\mathfrak{X}_{i}}, \ldots, R_{m}^{\mathfrak{X}_{i}}\right)$ with $R_{c}^{\mathfrak{X}_{i}}=\sigma_{i}^{-1}(c)$ can be regarded as a coloring of the edges of the complete directed graph on $B_{i}$ with $m$ colors. Since this coloring is compatible with the edge relation of $\mathfrak{B}_{i}$, the graph $\mathfrak{A}$ is a generalized product-in the sense of Feferman and Vaught - of the structures $\mathfrak{X}_{1}, \ldots, \mathfrak{X}_{n}$ using only atomic formulas.

We conclude by proving the "tamely-colorable" version of Theorem 3.3 .

Corollary 3.6. Given a tree-automatic $\tau$-structure $\mathfrak{A}$ and an $\mathrm{FO}[\tau]+\exists^{\infty}+\exists^{\text {chain }}$-formula $\varphi(x, \bar{y})$, one can compute a finite set $\mathcal{S}_{\varphi}^{\mathfrak{A}}$ of tree-automatic $\tau$-structures such that for all $\bar{s} \in A^{n}$ the substructure $\mathfrak{A} \uparrow_{\varphi, \bar{s}}$ is a sum-augmentation of tamely colorable box-augmentations of elements from $\mathcal{S}_{\varphi}^{\mathfrak{A}}$.

Proof. We show that the box-augmentation $\mathfrak{Z}$ of $\left(\mathfrak{X}_{u}\right)_{u \in U}$ constructed in the proof of Theorem 3.3 is tamely colorable. Therefore, consider the bijection $\eta: \prod_{u \in U} X_{u} \rightarrow Z$ witnessing this box-augmentation and fix some $R \in \mathcal{R}$ with arity $r=\operatorname{ar}(R)$. Due to the definition of $R^{\mathfrak{X}_{u}}$ in (3.2), for each $u \in U$ the map $\sigma_{u}: X_{u}^{r} \rightarrow Q_{R}$ with $\sigma_{u}(\bar{x})=\mathcal{A}_{R}(\otimes \bar{x})$ is a finite $R$-coloring of $\mathfrak{X}_{u}$. Finally, (3.8) shows that condition (2) from Definition 3.4 holds. 
3.2. Sum- and box-indecomposability. Suppose that $\mathcal{C}$ is a class of $\tau$-structures and $\nu$ is a function assigning an ordinal $\nu(\mathfrak{A})$ to each $\mathfrak{A} \in \mathcal{C} \mathbb{2}^{2}$ In this situation, we say that $\mathcal{C}$ is ranked by $\nu$.

Definition 3.7. Let $\mathcal{C}$ be a class of $\tau$-structures ranked by $\nu$.

(1) An ordinal $\alpha$ is called $\nu$-sum-indecomposable if for every $\mathfrak{A} \in \mathcal{C}$ with $\nu(\mathfrak{A})=\alpha$ and all $\tau$-structures $\mathfrak{B}_{1}, \ldots, \mathfrak{B}_{m}$ such that $\mathfrak{A}$ is a sum-augmentation of $\left(\mathfrak{B}_{1}, \ldots, \mathfrak{B}_{m}\right)$, there is $1 \leq i \leq m$ such that $\mathfrak{B}_{i} \in \mathcal{C}$ and $\nu\left(\mathfrak{B}_{i}\right)=\alpha$.

(2) An interval $\left[\alpha_{1}, \alpha_{2}\right]$ of ordinals is called $\nu$-tamely-colorable-box-indecomposable if for every $\mathfrak{A} \in \mathcal{C}$ with $\nu(\mathfrak{A})=\alpha_{2}$ and all $\tau$-structures $\mathfrak{B}_{1}, \ldots, \mathfrak{B}_{m}$ such that $\mathfrak{A}$ is a tamely colorable box-augmentation of $\left(\mathfrak{B}_{1}, \ldots, \mathfrak{B}_{m}\right)$, there is $1 \leq i \leq m$ such that $\mathfrak{B}_{i} \in \mathcal{C}$ and $\nu\left(\mathfrak{B}_{i}\right) \in\left[\alpha_{1}, \alpha_{2}\right]$.

Remark 3.8. For classes $\mathcal{C}$ which are closed under taking substructures, like the classes of forests and wulpo's, the requirement $\mathfrak{B}_{i} \in \mathcal{C}$ is always satisfied. Hence in this case explicitely requiring $\mathfrak{B}_{i} \in C$ is not necessary.

The decomposition results from the previous section imply that $\nu$ may only take finitely many $\nu$-sum-indecomposable and $\nu$-tamely-colorable-box-indecomposable values among the substructures of the form $\mathfrak{A} \uparrow_{\varphi, \bar{s}}$ (defined just before Theorem 3.3) for a fixed $\mathrm{FO}[\tau]+\exists^{\infty}+$ $\exists^{\text {chain }}$-formula $\varphi(x, \bar{y})$.

Proposition 3.9. Let $\mathcal{C}$ be a class of $\tau$-structures ranked by $\nu$ and $\alpha_{0}<\alpha_{1}<\alpha_{2}<\ldots$ an infinite sequence of $\nu$-sum-indecomposable ordinals such that $\left[\alpha_{i}, \alpha_{i+1}\right]$ is $\nu$-tamely-colorablebox-indecomposable for all $i \in \mathbb{N}$. Moreover, let $\mathfrak{A}$ be a tree-automatic $\tau$-structure and $\varphi\left(x, y_{1}, \ldots, y_{r}\right)$ an $\mathrm{FO}[\tau]+\exists^{\infty}+\exists^{\text {chain }}$-formula. Then there are only finitely many $i \in \mathbb{N}$ which admit a tuple $\bar{s} \in A^{r}$ with $\mathfrak{A} \uparrow_{\varphi, \bar{s}} \in \mathcal{C}$ and $\nu\left(\mathfrak{A} \uparrow_{\varphi, \bar{s}}\right)=\alpha_{i}$.

Proof. Let $\mathcal{S}_{\varphi}^{\mathfrak{A}}$ be the finite set of structures which exists by Corollary [3.6. Consider an $i \in \mathbb{N}_{>0}$ satisfying the condition above, witnessed by $\bar{s} \in A^{r}$, i.e., $\mathfrak{A} \uparrow_{\varphi, \bar{s}} \in \mathcal{C}$ and $\nu\left(\left.\mathfrak{A}\right|_{\varphi, \bar{s}}\right)=\alpha_{i}$. There are structures $\mathfrak{X}_{1}, \ldots, \mathfrak{X}_{k}$ such that each of them is a tamely colorable box-augmentation of elements from $\mathcal{S}_{\varphi}^{\mathfrak{A}}$ and $\mathfrak{A} \uparrow_{\varphi, \bar{s}}$ is a sum-augmentation of $\left(\mathfrak{X}_{1}, \ldots, \mathfrak{X}_{k}\right)$. Due to the definition of $\nu$-sum-indecomposability, there is a $1 \leq j \leq k$ such that $\mathfrak{X}_{j} \in \mathcal{C}$ and $\nu\left(\mathfrak{X}_{j}\right)=\alpha_{i}$. There are structures $\mathfrak{Y}_{1}, \ldots, \mathfrak{Y}_{\ell} \in \mathcal{S}_{\varphi}^{\mathfrak{A}}$ such that $\mathfrak{X}_{j}$ is a tamely colorable box-augmentation of $\left(\mathfrak{Y}_{1}, \ldots, \mathfrak{Y}_{\ell}\right)$. By the definition of $\nu$-tamely-colorable-boxindecomposability, there is an $1 \leq h \leq \ell$ such that $\mathfrak{Y}_{h} \in \mathcal{C}$ and $\nu\left(\mathfrak{Y}_{h}\right) \in\left[\alpha_{i-1}, \alpha_{i}\right]$. Thus, $i$ belongs to the set

$$
\left\{i \in \mathbb{N}_{>0} \mid \exists \mathfrak{B} \in \mathcal{S}_{\varphi}^{\mathfrak{A}} \cap \mathcal{C}: \nu(\mathfrak{B}) \in\left[\alpha_{i-1}, \alpha_{i}\right]\right\},
$$

which is finite since each $\mathfrak{B} \in \mathcal{S}_{\varphi}^{\mathfrak{A}}$ satisfies the condition above for at most two distinct $i$ 's. $\square$

\footnotetext{
${ }^{2}$ All our applications use isomorphism invariant functions, which means that $\nu(\mathfrak{A})=\nu(\mathfrak{B})$ if $\mathfrak{A} \cong \mathfrak{B}$. In fact, the results of this section apply to arbitrary class functions but are only useful if the function is isomorphism invariant for the important part of the class $\mathcal{C}$ because for other functions there are not enough indecomposable values. Thus, upon first reading of the following part, we recommend the reader to think of $\nu$ as an isomorphism invariant function.
} 
3.3. Rank-tamely-colorable-box- and rank-sum-indecomposability. Let us briefly prove that the ordinals $\omega^{\alpha}$ are rank-sum-indecomposable for the class of all well-founded partial orders. Afterwards, we characterize rank-tamely-colorable-box-indecomposable intervals for the classes of wulpo's and well-founded forests. For this purpose let rank ${ }_{u l}$ be the function obtained from rank by restriction of the domain to the class of wulpo's and analogously let rank $_{F}$ be rank restricted to the class of well-founded forests.

Proposition 3.10. The ordinals of the form $\omega^{\alpha}$ are rank-sum-indecomposable for the class of well-founded partial orders.

Proof. Let $\mathfrak{P}=(P, \leq)$ be a well-founded partial order and assume that $\mathfrak{P}$ is a sumaugmentation of $\left(\mathfrak{P}_{1}, \ldots, \mathfrak{P}_{n}\right)$. If $\operatorname{rank}\left(\mathfrak{P} \uparrow_{P \backslash P_{1}}\right)<\omega^{\alpha}$ and $\operatorname{rank}\left(\mathfrak{P}_{1}\right)<\omega^{\alpha}$, then

$$
\operatorname{rank}(\mathfrak{P}) \stackrel{\text { Lemma } 2.9}{\leq} \operatorname{rank}\left(\mathfrak{P}_{1}\right) \oplus \operatorname{rank}\left(\left.\mathfrak{P}\right|_{P \backslash P_{1}}\right)<\omega^{\alpha},
$$

where the last inequality follows from Property (2.2) of $\oplus$ (see page 6 ). Thus, $\operatorname{rank}(\mathfrak{P})=\omega^{\alpha}$ $\operatorname{implies} \operatorname{rank}\left(\mathfrak{P}_{1}\right)=\omega^{\alpha}$ or $\operatorname{rank}\left(\mathfrak{P} \uparrow_{P \backslash P_{1}}\right)=\omega^{\alpha}$. The claim follows by induction on $n$.

It follows trivially that the ordinals of the form $\omega^{\alpha}$ are rank $_{u l}$-sum-indecomposable and rank $_{F}$-sum-indecomposable.

We want to show that $\left[\omega^{\omega^{\alpha}}, \omega^{\omega^{\alpha+1}}\right]$ is a rank ul $_{\text {-tamely-colorable-box-indecomposable }}$ interval for each ordinal $\alpha$ and $\left[\omega^{\alpha}, \omega^{\alpha+1}\right]$ is a rank $F_{\text {-tamely-colorable-box-indecomposable }}$ interval for each ordinal $\alpha$. We start with the observation that every box-decomposition of a wulpo only contains at most one proper wulpo in the sense that if a wulpo is a boxaugmentation of $\left(\mathfrak{P}_{1}, \mathfrak{P}_{2}, \ldots, \mathfrak{P}_{n}\right)$ then all but one of the $\mathfrak{P}_{i}$ are disjoint unions of ordinals. In order to prove this fact, we introduce the following notation. Let $\mathfrak{P}=(P, \leq)$ be some partial order. We call $a \in P$ a branching node (of $\mathfrak{P}$ ), if there are $b, c \in P$ such that $b<a$, $c<a$ and neither $b \leq c$ nor $c \leq b$ (i.e., $b$ and $c$ are incomparable).

Lemma 3.11. Let $\mathfrak{P}, \mathfrak{P}_{1}$, and $\mathfrak{P}_{2}$ be wulpo's. If $\mathfrak{P}$ is a box-augmentation of $\left(\mathfrak{P}_{1}, \mathfrak{P}_{2}\right)$ then $\mathfrak{P}_{1}$ or $\mathfrak{P}_{2}$ does not contain a branching node.

Proof. Let $\mathfrak{P}=(P, \leq)$ and $\mathfrak{P}_{i}=\left(P_{i}, \leq_{i}\right)$. Heading for a contradiction assume that $a_{i}, b_{i}, c_{i} \in P_{i}$ for $i \in\{1,2\}$ are nodes such that $b_{i}<_{i} a_{i}, c_{i}<_{i} a_{i}$ and neither $b_{i} \leq_{i} c_{i}$ nor $c_{i} \leq_{i} b_{i}$.

Let $\eta: P_{1} \times P_{2} \rightarrow P$ be the bijection that witnesses that $\mathfrak{P}$ is a box-augmentation of $\left(\mathfrak{P}_{1}, \mathfrak{P}_{2}\right)$. Then $\mathfrak{P}$ contains the chains

$$
\begin{aligned}
& \eta\left(b_{1}, b_{2}\right)<\eta\left(b_{1}, a_{2}\right) \text { and } \\
& \eta\left(b_{1}, b_{2}\right)<\eta\left(a_{1}, b_{2}\right) .
\end{aligned}
$$

Since $\mathfrak{P}$ is a wulpo, the elements above $\eta\left(b_{1}, b_{2}\right)$ are linearly ordered and we may assume that $\eta\left(b_{1}, a_{2}\right)<\eta\left(a_{1}, b_{2}\right)$ without loss of generality. Thus, we obtain

$$
\eta\left(b_{1}, c_{2}\right)<\eta\left(b_{1}, a_{2}\right)<\eta\left(a_{1}, b_{2}\right) .
$$

Furthermore, we have

$$
\eta\left(b_{1}, c_{2}\right)<\eta\left(a_{1}, c_{2}\right)
$$

Again, the elements above $\eta\left(b_{1}, c_{2}\right)$ are linearly ordered and we obtain that $\eta\left(a_{1}, c_{2}\right)$ and $\eta\left(a_{1}, b_{2}\right)$ are comparable in $\mathfrak{P}$. By definition of a box-augmentation, we obtain that $c_{2}$ and $b_{2}$ are comparable in $\mathfrak{P}_{2}$ as well, which contradicts our assumptions. Thus, $\mathfrak{P}_{1}$ or $\mathfrak{P}_{2}$ does not contain a branching node. 
Corollary 3.12. Let $\mathfrak{P}$ and $\mathfrak{P}_{1}, \ldots, \mathfrak{P}_{n}$ be wulpo's such that $\mathfrak{P}$ is a box-augmentation of $\left(\mathfrak{P}_{1}, \ldots, \mathfrak{P}_{n}\right)$. There is at most one $i \in\{1, \ldots, n\}$ such that $\mathfrak{P}_{i}$ contains a branching node, i.e., there is an $i \in\{1, \ldots, n\}$ such that $\mathfrak{P}_{k}$ is a disjoint union of well-orders for all $k \neq i$.

Proof. Let $\eta: \prod_{i=1}^{n} \mathfrak{P}_{i} \rightarrow \mathfrak{P}$ be the bijection of the box-augmentation. Choose numbers $1 \leq j<k \leq n$ and a tuple $\bar{b}=\left(b_{1}, \ldots, b_{j-1}, b_{j+1}, \ldots, b_{k-1}, b_{k+1}, \ldots, b_{n}\right) \in \prod_{i \in\{1, \ldots n\} \backslash\{j, k\}} P_{i}$ arbitrarily but fixed. Then $\eta_{j, k}^{\bar{b}}: P_{j} \times P_{k} \rightarrow P$ with $\eta_{j, k}^{\bar{b}}\left(b_{j}, b_{k}\right)=\eta\left(b_{1}, \ldots, b_{n}\right)$ induces a box-augmentation of some subwulpo $\mathfrak{P}^{\prime} \leq \mathfrak{P}$. Application of Lemma 3.11 yields the claim.

Remark 3.13. In the following, our proofs for the case of wulpo's and the case of wellfounded forests proceed completely analogous. The difference in the results stems from an observation concerning Corollary 3.12 ; if a well-founded forest $\mathfrak{F}$ is a box-augmentation of partial orders $\mathfrak{P}_{1}, \ldots, \mathfrak{P}_{n}$ then the $\mathfrak{P}_{i}$ occur as substructures of $\mathfrak{F}$. Hence, these are also well-founded forests. But if a well-founded forest is a disjoint union of ordinals, all these ordinals must be finite. Thus, each connected component of such a disjoint union has finite rank and the whole structure has rank at most $\omega$. In contrast, if a disjoint union of ordinals is a tree-automatic wulpo each connected component is a tree-automatic ordinal whence its rank is bounded by $\omega^{\omega^{\omega}}$ and all smaller ordinals can be reached. This difference causes the different results with respect to box-indecomposability.

Lemma 3.14. Let $\mathfrak{P}$ and $\mathfrak{P}_{1}, \ldots, \mathfrak{P}_{m}$ be wulpo's such that $\mathfrak{P}$ is a box-augmentation of $\left(\mathfrak{P}_{1}, \ldots, \mathfrak{P}_{m}\right)$ via $\eta: \prod_{i=1}^{m} \mathfrak{P}_{i} \rightarrow \mathfrak{P}$. Let $I_{i} \subseteq P_{i}$ be a well-order. The substructure $\mathfrak{P}^{\prime} \subseteq \mathfrak{P}$ induced by $\eta\left(\prod_{i=1}^{m} I_{i}\right)$ is a well-order.

Proof. Note that $\mathfrak{P}^{\prime}$ is a wulpo because it is a substructure of $\mathfrak{P}$. Thus, it suffices to show that $\mathfrak{P}^{\prime}$ is linear. Let $a_{i}^{j} \in I_{i}$ for each $1 \leq i \leq m$ and $j \in\{1,2\}$. Set $m_{i}=\min \left(a_{i}^{1}, a_{i}^{2}\right)$. For $j \in\{1,2\}$,

$$
\eta\left(m_{1}, m_{2}, \ldots, m_{n}\right) \leq \eta\left(a_{1}^{j}, m_{2}, \ldots, m_{n}\right) \leq \cdots \leq \eta\left(a_{1}^{j}, a_{2}^{j}, \ldots, a_{n}^{j}\right) .
$$

Since the elements above $\eta\left(m_{1}, \ldots, m_{n}\right)$ are pairwise comparable, the elements $\eta\left(a_{1}^{1}, \ldots, a_{n}^{1}\right)$ and $\eta\left(a_{1}^{2}, \ldots, a_{n}^{2}\right)$ are comparable. Since the $a_{i}^{j}$ have been chosen arbitrarily any two elements of $\mathfrak{P}^{\prime}$ are comparable, i.e., $\mathfrak{P}^{\prime}$ is linear.

In the following lemma, the term "replacement" refers to the replacement operation introduced at the end of Section 2.1.

Lemma 3.15. If a wulpo $\mathfrak{P}$ is the box-augmentation of ordinals $\mathfrak{C}_{1}, \ldots, \mathfrak{C}_{n}$ and a wulpo $\mathfrak{Q}$ via the bijection $\eta$, then $\mathfrak{P}$ results from $\mathfrak{Q}$ by replacing every maximal branching free interval I of $\mathfrak{Q}$ by $\eta\left(\left(\prod_{i=1}^{n} \mathfrak{C}_{i}\right) \times I\right)$ (which is a well-order by Lemma 3.14).

Proof. We first show the following two claims.

(1) For all $q_{0}, q_{1} \in \mathfrak{Q}$ and for all $b_{i}, c_{i} \in \mathfrak{C}_{i}$ the nodes $\eta\left(b_{1}, \ldots, b_{n}, q_{0}\right)$ and $\eta\left(c_{1}, \ldots, c_{n}, q_{1}\right)$ are incomparable if and only if $q_{0}$ and $q_{1}$ are incomparable.

(2) For all $b_{i}, c_{i} \in \mathfrak{C}_{i}$ and $q_{0}, q_{1}, q \in \mathfrak{Q}$ such that $q_{0}<q$ and $q_{1}<q$ but $q_{0}$ and $q_{1}$ are incomparable, $\eta\left(b_{1}, \ldots, b_{n}, q_{1}\right)<\eta\left(c_{1}, \ldots, c_{n}, q\right)$.

The first statement is easy to show: If $\eta\left(b_{1}, \ldots, b_{n}, q_{0}\right) \leq \eta\left(c_{1}, \ldots, c_{n}, q_{1}\right)$ then the elements $\eta\left(\max \left(b_{1}, c_{1}\right), \ldots, \max \left(b_{n}, c_{n}\right), q_{0}\right)$ and $\eta\left(\max \left(b_{1}, c_{1}\right), \ldots, \max \left(b_{n}, c_{n}\right), q_{1}\right)$ are both above the 
element $\eta\left(b_{1}, \ldots, b_{n}, q_{0}\right)$ and therefore comparable. By the definition of a box-augmentation, also $q_{0}$ and $q_{1}$ are comparable. For the other direction, if $q_{0} \leq q_{1}$, then

$$
\eta\left(\min \left(b_{1}, c_{1}\right), \ldots, \min \left(b_{n}, c_{n}\right), q_{0}\right) \leq \eta\left(\min \left(b_{1}, c_{1}\right), \ldots, \min \left(b_{n}, c_{n}\right), q_{1}\right) .
$$

Thus, $\eta\left(b_{1}, \ldots, b_{n}, q_{0}\right)$ and $\eta\left(c_{1}, \ldots, c_{n}, q_{1}\right)$ are both above $\eta\left(\min \left(b_{1}, c_{1}\right), \ldots, \min \left(b_{n}, c_{n}\right), q_{0}\right)$ and therefore comparable.

Let us now prove the second claim. Due to the first claim, $q_{1}<q$ implies that $\eta\left(b_{1}, \ldots, b_{n}, q_{1}\right)$ and $\eta\left(c_{1}, \ldots, c_{n}, q\right)$ are comparable. Moreover,

$$
\text { for all } a_{i} \in \mathfrak{C}_{i}, \eta\left(a_{1}, \ldots, a_{n}, q_{0}\right) \text { and } \eta\left(a_{1}, \ldots, a_{n}, q_{1}\right) \text { are incomparable, }
$$

because $q_{0}$ and $q_{1}$ are incomparable and $\mathfrak{P}$ is a box-augmentation. Similarly, it is clear that $\eta\left(\min \left(b_{1}, c_{1}\right), \ldots, \min \left(b_{n}, c_{n}\right), q_{i}\right) \leq \eta\left(\min \left(b_{1}, c_{1}\right), \ldots, \min \left(b_{n}, c_{n}\right), q\right) \leq \eta\left(c_{1}, \ldots, c_{n}, q\right)$ and $\eta\left(\min \left(b_{1}, c_{1}\right), \ldots, \min \left(b_{n}, c_{n}\right), q_{i}\right) \leq \eta\left(b_{1}, \ldots, b_{n}, q_{i}\right)$

for $i \in\{0,1\}$. Since $\mathfrak{P}$ is a wulpo, the nodes above $\eta\left(\min \left(b_{1}, c_{1}\right), \ldots, \min \left(b_{n}, c_{n}\right), q_{i}\right)$ are linearly ordered. Hence $\eta\left(c_{1}, \ldots, c_{n}, q\right)$ and $\eta\left(b_{1}, \ldots, b_{n}, q_{i}\right)$ are comparable for $i \in\{0,1\}$. Note that

$$
\eta\left(b_{1}, \ldots, b_{n}, q_{i}\right) \leq \eta\left(c_{1}, \ldots, c_{n}, q\right) \leq \eta\left(b_{1}, \ldots, b_{n}, q_{1-i}\right)
$$

for $i \in\{0,1\}$ would contradict (3.9). Analogously, if

$$
\begin{aligned}
& \eta\left(c_{1}, \ldots, c_{n}, q\right) \leq \eta\left(b_{1}, \ldots, b_{n}, q_{1}\right) \text { and } \\
& \eta\left(c_{1}, \ldots, c_{n}, q\right) \leq \eta\left(b_{1}, \ldots, b_{n}, q_{0}\right),
\end{aligned}
$$

then upwards linearity would lead to a contradiction with (3.9). Thus, we conclude that

$$
\eta\left(b_{1}, \ldots, b_{n}, q_{1}\right) \leq \eta\left(c_{1}, \ldots, c_{n}, q\right),
$$

which shows the second claim.

Using these two claims, we now show that for every pair $I_{1}, I_{2}$ of distinct maximal branching free intervals of $\mathfrak{Q}$ and for all $q_{1} \in I_{1}, q_{2} \in I_{2}$, and for all $b_{i}, c_{i} \in \mathfrak{C}_{i}$ we have

$$
q_{1}<q_{2} \Longleftrightarrow \eta\left(b_{1}, \ldots, b_{n}, q_{1}\right)<\eta\left(c_{1}, \ldots, c_{n}, q_{2}\right) .
$$

First assume that $q_{1}<q_{2}$. Since $q_{1}$ and $q_{2}$ come from different maximal branching free intervals, Lemma 2.5 implies that there is a $q_{3}<q_{2}$ such that $q_{1}$ and $q_{3}$ are incomparable. Due to the second claim, this immediately shows that $\eta\left(b_{1}, \ldots, b_{n}, q_{1}\right)<\eta\left(c_{1}, \ldots, c_{n}, q_{2}\right)$. By symmetry, we conclude that $q_{2}<q_{1}$ implies $\eta\left(b_{1}, \ldots, b_{n}, q_{2}\right)<\eta\left(c_{1}, \ldots, c_{n}, q_{1}\right)$. Moreover, because of the first claim, if $q_{1}$ and $q_{2}$ are incomparable then $\eta\left(b_{1}, \ldots, b_{n}, q_{1}\right)$ and $\eta\left(c_{1}, \ldots, c_{n}, q_{2}\right)$ are incomparable as well.

By (3.10), $\mathfrak{P}$ is obtained from $\mathfrak{Q}$ by replacing every maximal branching free interval $I$ of $\mathfrak{Q}$ by a box-augmentation of $\left(\mathfrak{C}_{1}, \ldots, \mathfrak{C}_{n}, I\right)$, which is a well order by Lemma 3.14. This concludes the proof of the lemma.

Lemma 3.16. Let $\mathfrak{P}$ be a wulpo that is a box-augmentation of ordinals $\mathfrak{C}_{1}, \ldots, \mathfrak{C}_{n}$ and a wulpo $\mathfrak{Q}$ via the bijection $\eta$. Let $q \in \mathfrak{Q}$ and $q^{\prime} \in \mathfrak{Q} \cup\{\infty\}$ such that $q<q^{\prime}$ and $\left[q, q^{\prime}\right)$ is a maximal branching free interval in $\mathfrak{Q}$. Let $\alpha:=\operatorname{rank}\left(\left[q, q^{\prime}\right)\right)>0$ and $0_{i}$ the minimal element of $\mathfrak{C}_{i}$ for each $1 \leq i \leq n$. Then

$$
\operatorname{rank}\left(\eta\left(0_{1}, 0_{2}, \ldots, 0_{n}, q\right), \mathfrak{P}\right) \leq \operatorname{rank}\left(\mathfrak{C}_{1}\right) \otimes \operatorname{rank}\left(\mathfrak{C}_{2}\right) \otimes \cdots \otimes \operatorname{rank}\left(\mathfrak{C}_{n}\right) \otimes \operatorname{rank}(q, \mathfrak{Q})
$$

and for each $\hat{q} \in\left[q, q^{\prime}\right)$ and $c_{i} \in \mathfrak{C}_{i}$ we have

$$
\operatorname{rank}\left(\eta\left(c_{1}, c_{2}, \ldots, c_{n}, \hat{q}\right), \mathfrak{P}\right)<\operatorname{rank}\left(\mathfrak{C}_{1}\right) \otimes \operatorname{rank}\left(\mathfrak{C}_{2}\right) \otimes \cdots \otimes \operatorname{rank}\left(\mathfrak{C}_{n}\right) \otimes(\operatorname{rank}(q, \mathfrak{Q})+\alpha) .
$$


Proof. We prove both claims simultaneously by induction. If $\operatorname{rank}(q, \mathfrak{Q})=0$, then $q$ is minimal in $\mathfrak{Q}$. By Lemma 3.15, $\eta\left(0_{1}, 0_{2}, \ldots, 0_{n}, q\right)$ is minimal in $\mathfrak{P}$. Hence, its rank is also 0 as desired.

Now assume that (3.11) is true for some $q \in \mathfrak{Q}$ inducing a maximal branching free interval $\left[q, q^{\prime}\right) \subseteq \mathfrak{Q}$ and let $c_{i} \in \mathfrak{C}_{i}$ and $\hat{q} \in\left[q, q^{\prime}\right)$. Due to Lemma 3.15, $I:=\eta\left(\prod_{i=1}^{n} \mathfrak{C}_{i} \times\right.$ $\left.\left[q, q^{\prime}\right)\right)$ is a maximal branching free interval of $\mathfrak{P}$ with minimal element $\eta\left(0_{1}, 0_{2}, \ldots, 0_{n}, q\right)$. The rank of $I$ can be at most $\operatorname{rank}\left(\mathfrak{C}_{1}\right) \otimes \cdots \otimes \operatorname{rank}\left(\mathfrak{C}_{n}\right) \otimes \alpha$. The interval

$$
J:=\left[\eta\left(0_{1}, \ldots, 0_{n}, q\right), \eta\left(c_{1}, \ldots, c_{n}, \hat{q}\right)\right) \subseteq \mathfrak{P}
$$

is strictly contained in $I$ whence

$$
\operatorname{rank}(J)<\operatorname{rank}\left(\mathfrak{C}_{1}\right) \otimes \cdots \otimes \operatorname{rank}\left(\mathfrak{C}_{n}\right) \otimes \alpha .
$$

Thus, we get

$$
\begin{aligned}
& \quad \operatorname{rank}\left(\eta\left(c_{1}, \ldots, c_{n}, \hat{q}\right), \mathfrak{P}\right) \\
& \frac{\text { Lem. }}{=} \operatorname{rank}\left(\eta\left(0_{1}, \ldots, 0_{n}, q\right), \mathfrak{P}\right)+\operatorname{rank}(J) \\
& \frac{2.10}{\left(\frac{3.13}{<}\right.} \operatorname{rank}\left(\eta\left(0_{1}, \ldots, 0_{n}, q\right), \mathfrak{P}\right)+\operatorname{rank}\left(\mathfrak{C}_{1}\right) \otimes \cdots \otimes \operatorname{rank}\left(\mathfrak{C}_{n}\right) \otimes \alpha \\
& \stackrel{(\operatorname{3.11})}{\leq}\left(\operatorname{rank}\left(\mathfrak{C}_{1}\right) \otimes \cdots \otimes \operatorname{rank}\left(\mathfrak{C}_{n}\right) \otimes \operatorname{rank}(q, \mathfrak{Q})\right)+\left(\operatorname{rank}\left(\mathfrak{C}_{1}\right) \otimes \cdots \otimes \operatorname{rank}\left(\mathfrak{C}_{n}\right) \otimes \alpha\right) \\
& \text { Lem. } \\
& \leq \operatorname{rank}\left(\mathfrak{C}_{1}\right) \otimes \cdots \otimes \operatorname{rank}\left(\mathfrak{C}_{n}\right) \otimes(\operatorname{rank}(q, \mathfrak{Q})+\alpha) .
\end{aligned}
$$

Finally, let $q, q^{\prime} \in \mathfrak{Q}$ be nodes such that $\left[q, q^{\prime}\right)$ is maximal branching free and all maximal branching free intervals below $q$ satisfy the claims. Lemma 2.4 shows that for each $q_{1}<q$ there is a maximal branching free interval $I:=\left[q_{0}, q_{2}\right)$ with $q_{2} \leq q$ such that $q_{1} \in I$. Then for all $c_{i} \in \mathfrak{C}_{i}$

$$
\begin{aligned}
& \operatorname{rank}\left(\eta\left(c_{1}, c_{2}, \ldots, c_{n}, q_{1}\right), \mathfrak{P}\right) \stackrel{\mathrm{IH}}{<} \operatorname{rank}\left(\mathfrak{C}_{1}\right) \otimes \cdots \otimes \operatorname{rank}\left(\mathfrak{C}_{n}\right) \otimes\left(\operatorname{rank}\left(q_{0}, \mathfrak{Q}\right)+\operatorname{rank}(I)\right) \\
& \frac{\mathrm{Lem.}}{\leq} \operatorname{rank}\left(\mathfrak{C}_{1}\right) \otimes \cdots \otimes \operatorname{rank}\left(\mathfrak{C}_{n}\right) \otimes\left(\operatorname{rank}\left(q_{2}, \mathfrak{Q}\right)\right. \\
& \frac{2.10}{\leq} \operatorname{rank}\left(\mathfrak{C}_{1}\right) \otimes \cdots \otimes \operatorname{rank}\left(\mathfrak{C}_{n}\right) \otimes(\operatorname{rank}(q, \mathfrak{Q}) .
\end{aligned}
$$

By Lemma 3.15 all elements below $\eta\left(0_{1}, 0_{2}, \ldots, 0_{n}, q\right)$ are of the form $\eta\left(c_{1}, c_{2}, \ldots, c_{n}, q_{1}\right)$ with $q_{1}<q$. Thus,

$$
\operatorname{rank}\left(\eta\left(0_{1}, 0_{2}, \ldots, 0_{n}, q\right) \leq \operatorname{rank}\left(\mathfrak{C}_{1}\right) \otimes \cdots \otimes \operatorname{rank}\left(\mathfrak{C}_{n}\right) \otimes(\operatorname{rank}(q, \mathfrak{Q}),\right.
$$

which proves the lemma.

Corollary 3.17. Assume that $\mathfrak{P}$ is a wulpo that is a box-augmentation of connected wulpo's $\mathfrak{Q}_{1}, \ldots, \mathfrak{Q}_{n}$. Then

$$
\operatorname{rank}(\mathfrak{P}) \leq \operatorname{rank}\left(\mathfrak{Q}_{1}\right) \otimes \cdots \otimes \operatorname{rank}\left(\mathfrak{Q}_{n}\right) .
$$

Proof. By Corollary 3.12 we can assume that $\mathfrak{Q}_{2}, \ldots, \mathfrak{Q}_{n}$ are ordinals with minimal elements $0_{2}, \ldots, 0_{n}$. For each node $p \in \mathfrak{P}$ there is a maximal branching free interval $\left[p_{0}, p_{1}\right) \subseteq \mathfrak{P}$ such that $p \in\left[p_{0}, p_{1}\right)$ (where $p_{1}=\infty$ is possible). There is a $q_{0} \in \mathfrak{Q}_{1}$ such that $p_{0}=$ 
$\eta\left(q_{0}, 0_{2}, \ldots, 0_{n}\right)$ and there is a $q_{1} \in \mathfrak{Q}_{1} \cup\{\infty\}$ such that $I:=\left[q_{0}, q_{1}\right)$ is maximal branching free. By Lemma 3.16, we have

$$
\begin{aligned}
\operatorname{rank}(p, \mathfrak{P}) & <\operatorname{rank}\left(\mathfrak{Q}_{2}\right) \otimes \cdots \otimes \operatorname{rank}\left(\mathfrak{Q}_{n}\right) \otimes\left(\operatorname{rank}\left(q_{0}, \mathfrak{Q}_{1}\right)+\operatorname{rank}(I)\right) \\
& \leq \operatorname{rank}\left(\mathfrak{Q}_{2}\right) \otimes \cdots \otimes \operatorname{rank}\left(\mathfrak{Q}_{n}\right) \otimes \operatorname{rank}\left(\mathfrak{Q}_{1}\right),
\end{aligned}
$$

where in case that $p=p_{0}$ the strict inequality follows from $\operatorname{rank}(I) \geq 1$.

Having studied connected wulpo's, we now have to deal with disconnected ones. For this purpose, we have to restrict our attention to tamely colorable box-augmentations. As a first step, we analyze boxes of antichains. If a wulpo $\mathfrak{P}$ is a tamely colorable box-augmentation of $n$ antichains then the rank of $\mathfrak{P}$ is bounded by some constant that only depends on the tame colorings of the antichains and on $n$. In order to prove this fact, we first introduce a notion of same factor equivalence on the elements of a box-augmentation. Elements are equivalent with respect to this equivalence if and only if their preimages in each of the factors of the box are contained in the same connected component.

Definition 3.18. Let $\mathfrak{P}$ be a wulpo that is a box-augmentation of (wulpo's) $\left(\mathfrak{P}_{1}, \ldots, \mathfrak{P}_{n}\right)$. Let $\mathfrak{P}_{i}=\left(P_{i}, \leq_{i}\right)$. For $a_{i}, b_{i} \in P_{i}$ we write $a_{i} \equiv_{i} b_{i}$ if $a_{i}$ and $b_{i}$ are in the same connected component of $\mathfrak{P}_{i}$. Due to upwards linearity, this is equivalent to saying that $a_{i} \equiv_{i} b_{i}$ if there exists some $c_{i} \in P_{i}$ such that $a_{i} \leq_{i} c_{i}$ and $b_{i} \leq_{i} c_{i}$.

Let $\eta$ be the bijection witnessing that $\mathfrak{P}$ is box-augmentation of $\left(\mathfrak{P}_{1}, \ldots, \mathfrak{P}_{n}\right)$. For $a, b \in P$ we define the same factors equivalence by $a \equiv b$ if $a_{i} \equiv_{i} b_{i}$ for all $1 \leq i \leq n$ where $a=\eta\left(a_{1}, \ldots, a_{n}\right)$ and $b=\eta\left(b_{1}, \ldots, b_{n}\right)$. For $p \in P$ we write $[p]_{\equiv}$ for the equivalence class $\left\{p^{\prime} \mid p^{\prime} \equiv p\right\}$.

Lemma 3.19. Let $\mathfrak{P}=(P, \leq)$ be a wulpo that is a box-augmentation of $\left(\mathfrak{P}_{1}, \ldots, \mathfrak{P}_{n}\right)$ via the bijection $\eta$. If $C \subseteq P$ is a chain that contains elements of $k$ distinct $\equiv$-classes, then $\mathfrak{P}$ contains a chain $C^{\prime}$ of length $k$ such that $C^{\prime} \subseteq \eta\left(A_{1} \times \cdots \times A_{n}\right)$ where $A_{i}$ is an antichain in $\mathfrak{P}_{i}$.

Proof. Let $a^{1}<a^{2}<\cdots<a^{k}$ be a chain of pairwise $\equiv$-inequivalent elements of $\mathfrak{P}$. There are $a_{i}^{j} \in \mathfrak{P}_{i}$ such that $a^{j}=\eta\left(a_{1}^{j}, \ldots, a_{n}^{j}\right)$. Let

$$
A_{i}^{j}:=\left\{a_{i}^{j^{\prime}} \mid a_{i}^{j} \equiv_{i} a_{i}^{j^{\prime}}\right\}
$$

be the connected component of $a_{i}^{j}$ restricted to those elements that appear as factors of the $a^{j^{\prime}}$ with $1 \leq j, j^{\prime} \leq k$. Due to upwards linearity, for all $1 \leq i \leq n$ and $1 \leq j \leq k$ there is a minimal $m_{i}^{j} \in P_{i}$ such that for all $x \in A_{i}^{j}, x \leq_{i} m_{i}^{j}$. By definition, we have

$$
m_{i}^{j}=m_{i}^{j^{\prime}} \Leftrightarrow a_{i}^{j} \equiv_{i} a_{i}^{j^{\prime}} \Leftrightarrow A_{i}^{j}=A_{i}^{j^{\prime}} .
$$

By definition of a box-augmentation we have

$$
a^{j}=\eta\left(a_{1}^{j}, \ldots, a_{n}^{j}\right) \leq \eta\left(m_{1}^{j}, a_{2}^{j}, \ldots, a_{n}^{j}\right) \leq \cdots \leq \eta\left(m_{1}^{j}, \ldots, m_{n}^{j}\right) .
$$

Thus, $a^{1} \leq a^{j} \leq \eta\left(m_{1}^{j}, \ldots, m_{n}^{j}\right)$ for all $1 \leq j \leq k$. Due to upwards linearity, the set

$$
C^{\prime}:=\left\{\eta\left(m_{1}^{j}, \ldots, m_{n}^{j}\right) \mid 1 \leq j \leq k\right\}
$$

forms a chain. Note that $\eta\left(m_{1}^{j}, \ldots, m_{n}^{j}\right)=\eta\left(m_{1}^{j^{\prime}}, \ldots, m_{n}^{j^{\prime}}\right)$ would imply $m_{i}^{j}=m_{i}^{j^{\prime}}$, i.e., $a_{i}^{j} \equiv_{i} a_{i}^{j^{\prime}}$ (by (3.14) $)$ for all $1 \leq i \leq n$. But this would lead to the contradiction $a^{j} \equiv a^{j^{\prime}}$. Thus, the chain $C^{\prime}$ consists of $k$ elements. We conclude by proving that $A_{i}:=\left\{m_{i}^{j} \mid 1 \leq\right.$ 
$j \leq k\}$ is an antichain in $\mathfrak{P}_{i}$. Heading for a contradiction assume that there are $j \neq j^{\prime}$ such that $m_{i}^{j}<m_{i}^{j^{\prime}}$. Then clearly $m_{i}^{j} \equiv_{i} m_{i}^{j^{\prime}}$ holds and hence also $a_{i}^{j} \equiv_{i} a_{i}^{j^{\prime}}$ holds. With (3.14) we conclude that $m_{i}^{j}=m_{i}^{j^{\prime}}$ contradicting our assumption $m_{i}^{j}<m_{i}^{j^{\prime}}$.

The previous result can be used to bound the length of ordinals occurring in the image of antichains in a tamely colorable box-augmentation.

Lemma 3.20. Let $\mathfrak{P}=(P, \leq)$ be a wulpo which is a tamely colorable box-augmentation of $\left(\mathfrak{P}_{1}, \mathfrak{P}_{2}, \ldots, \mathfrak{P}_{n}\right)$ via $\eta$. There is a constant $c \in \mathbb{N}$ such that the following holds: For all choices of antichains $A_{i} \subseteq P_{i}$ (for each $1 \leq i \leq n$ ), the substructure of $\mathfrak{P}$ induced by $\eta\left(\prod_{i=1}^{n} A_{i}\right)$ does not contain a chain of length $c$.

Proof. For each $1 \leq i \leq n$ we fix a finite coloring $\sigma_{i}: P_{i} \times P_{i} \rightarrow C_{i}$ of $\mathfrak{P}_{i}$ such that the map $\sigma: P \times P \rightarrow C$ with $C=\prod_{1 \leq i \leq n} C_{i}$ and

$$
\sigma\left(\eta\left(p_{1}, \ldots, p_{n}\right), \eta\left(q_{1}, \ldots, q_{n}\right)\right)=\left(\sigma_{1}\left(p_{1}, q_{1}\right), \ldots, \sigma_{n}\left(p_{n}, q_{n}\right)\right) .
$$

is a finite coloring of $\mathfrak{P}$.

By Ramsey's theorem [23] there exists a constant $c \in \mathbb{N}$ such that every complete simple graph with at least $c$ nodes whose edges are colored by $|C|$ colors contains a monochromatic triangle.

For the sake of a contradiction, assume that for each $1 \leq i \leq n$ there exists an antichain $A_{i}$ in $\mathfrak{P}_{i}$ such that $\eta\left(\prod_{i=1}^{n} A_{i}\right)$ contains a chain of length $c$. Due to the choice of $c$ there exist three elements $p^{1}>p^{2}>p^{3}$ in this chain and a color $\bar{c}=\left(c_{1}, \ldots, c_{n}\right) \in C$ such that

$$
\sigma\left(p^{1}, p^{2}\right)=\sigma\left(p^{1}, p^{3}\right)=\sigma\left(p^{2}, p^{3}\right)=\bar{c} .
$$

Let $p^{j}=\eta\left(p_{1}^{j}, \ldots, p_{n}^{j}\right)$, where $p_{i}^{j} \in A_{i}$. For each $1 \leq i \leq n$ we obtain

$$
\sigma_{i}\left(p_{i}^{1}, p_{i}^{2}\right)=\sigma_{i}\left(p_{i}^{1}, p_{i}^{3}\right)=\sigma_{i}\left(p_{i}^{2}, p_{i}^{3}\right)=c_{i} .
$$

For $p^{\prime}=\eta\left(p_{1}^{2}, p_{2}^{1}, \ldots, p_{n}^{1}\right)$ we get $\sigma\left(p^{1}, p^{3}\right)=\sigma\left(p^{\prime}, p^{3}\right)$. Since $p^{1}>p^{3}$, this implies $p^{\prime}>p^{3}$. As the elements above $p^{3}$ are linearly ordered, we conclude that $p^{\prime}$ and $p^{1}$ are comparable. Recall that $\eta\left(\cdot, p_{2}^{1}, \ldots p_{n}^{1}\right)$ embeds $\mathfrak{P}_{1}$ into $\mathfrak{P}$. We conclude that $p_{1}^{1}$ and $p_{1}^{2}$ are comparable in $\mathfrak{P}_{1}$. Since $A_{1}$ is an antichain and $p_{1}^{1}, p_{1}^{2} \in A_{1}$, this implies $p_{1}^{1}=p_{1}^{2}$.

Analogous arguments for the other coordinates show that $p_{i}^{1}=p_{i}^{2}$ for each $1 \leq i \leq n$, i.e., $p^{1}=p^{2}$. However, this contradicts $p^{1}>p^{2}$.

We now head for the following result. Given a wulpo that is a tamely colorable box-augmentation we can write it as a finite sum-augmentation of wulpo's whose rank is bounded in terms of the ranks of the connected components of the factors of the box.

Lemma 3.21. Let $\mathfrak{P}=(P, \leq)$ be a countable wulpo which is a tamely colorable box augmentation of $\left(\mathfrak{P}_{1}, \ldots, \mathfrak{P}_{n}\right)$ via the bijection $\eta$. Let $c \in \mathbb{N}$ such that the image of antichains under $\eta$ does not contain a chain of length $c$ (exists by Lemma 3.20). Then there is a map $\mu: P \rightarrow\{1, \ldots, c\}$ such that for all chains $L \subseteq P$, and all $p, p^{\prime} \in L$ we have $\mu(p)=\mu\left(p^{\prime}\right)$ if and only if $p^{\prime} \equiv p$.

Proof. Fix an enumeration $m_{1}, m_{2}, \ldots$ of the minimal elements of $\mathfrak{P}$. Let $\mu_{0}$ be the partial function with empty domain. We define $\mu$ as the limit of partial functions $\mu_{i}$ satisfying the lemma (restricted to their domain). This limit $\mu$ is a total function because we guarantee 
that

$$
\operatorname{dom}\left(\mu_{i}\right)=\bigcup_{j=1}^{i}\left[m_{j}, \infty\right)
$$

Assume that $\mu_{j}$ has already been defined.

First we set $\mu_{j+1}(x)=\mu_{j}(x)$ for all $x \in \operatorname{dom}\left(\mu_{j}\right)$. By transfinite induction we extend $\mu_{j+1}$ to all nodes $y \in\left[m_{j+1}, \infty\right) \backslash \bigcup_{i=1}^{j}\left[m_{i}, \infty\right)$. For this purpose assume that $y \in\left[m_{j+1}, \infty\right) \backslash$ $\bigcup_{i=1}^{j}\left[m_{i}, \infty\right)$ and assume that we have defined $\mu_{j+1}(x)$ for all $m_{j+1} \leq x<y$. Hence, the domain of the current $\mu_{j+1}$ is

$$
D=\bigcup_{i=1}^{j}\left[m_{i}, \infty\right) \cup\left[m_{j+1}, y\right) .
$$

We assume that for all chains $L \subseteq D$, and all $p, p^{\prime} \in L$ we have $\mu_{j+1}(p)=\mu_{j+1}\left(p^{\prime}\right)$ if and only if $p^{\prime} \equiv p$. Let

$$
J=\{1, \ldots, c\} \backslash \mu_{j+1}\left(D \cap\left[m_{j+1}, \infty\right)\right) .
$$

Then $J \neq \emptyset: D \cap\left[m_{j+1}, \infty\right)$ is a chain. By Lemma 3.19 and the choice of $c$, each chain in $\mathfrak{P}$ contains elements of at most $c-1$ many $\equiv$-classes. Hence, the elements from $D \cap\left[m_{j+1}, \infty\right)$ fall into at most $c-1$ many $\equiv$-classes. By our assumption on the current $\mu_{j+1}$, this mapping takes at most $c-1$ different values among the elements from $D \cap\left[m_{j+1}, \infty\right)$. Hence, $J$ is not empty.

Now we extend $\mu_{j+1}$ to $y$ as follows:

$$
\mu_{j+1}(y):= \begin{cases}\mu_{j+1}(p) & \text { if } p \in D \cap\left[m_{j+1}, \infty\right) \text { and } p \equiv y \\ \min (J) & \text { otherwise. }\end{cases}
$$

Each of the $\mu_{j}$ is a well-defined partial function and setting $\mu=\bigcup_{j \in \mathbb{N}} \mu_{j}$ settles the claim.

Corollary 3.22. Let $\mathfrak{P}, c$, and $\mu$ be defined as in Lemma 3.21. Let $1 \leq i \leq c$ and $a, b \in \mu^{-1}(i)$ such that $a$ and $b$ are in a connected component of the suborder induced by $\mu^{-1}(i) \subseteq \mathfrak{P}$. Then we have $a \equiv b$.

Proof. Let $\mu(a)=i=\mu(b)$ and assume that $a$ and $b$ are in a connected component of the suborder induced by $\mu^{-1}(i) \subseteq \mathfrak{P}$. Since this suborder is again a wulpo, there exists $p \in \mu^{-1}(i)$ with $a \leq p$ and $b \leq p$. Lemma 3.21 together with $\mu(a)=\mu(p)=\mu(b)$ implies $a \equiv p \equiv b$, i.e., $a \equiv b$ by transitivity of $\equiv$.

Corollary 3.23. Let $\mathfrak{P}$ be a wulpo that is box-augmentation of (wulpo's) $\left(\mathfrak{P}_{1}, \mathfrak{P}_{2}, \ldots, \mathfrak{P}_{m}\right)$ such that there is some $n \in \mathbb{N}$ with $\operatorname{rank}\left(\mathfrak{P}_{j}\right)<\omega^{\omega^{n}}$ for all $1 \leq j \leq m$.

(1) For $\mu$ as in Lemma 3.21, $\operatorname{rank}\left(\mathfrak{P} \uparrow_{\mu^{-1}(i)}\right) \leq \omega^{\omega^{n}}$ for all $i$ in the range of $\mu$.

(2) Moreover, if $\mathfrak{P}$ is a forest and there is $n \in \mathbb{N}$ with $\operatorname{rank}\left(\mathfrak{P}_{j}\right)<\omega^{n}$ for all $1 \leq j \leq m$, then $\operatorname{rank}\left(\mathfrak{P} \uparrow_{\mu^{-1}(i)}\right) \leq \omega^{n}$ for all $i$ in the range of $\mu$.

Proof. Let $\mathfrak{C}_{j}$ be the set of connected components of $\mathfrak{P}_{j}$ for all $1 \leq j \leq m$. Due to Corollary 3.22, $\mathfrak{P} \uparrow_{\mu^{-1}(i)}$ is isomorphic to some suborder of the disjoint union

$$
\left.\bigsqcup_{\left(D_{1}, \ldots, D_{m}\right) \in \mathfrak{C}_{1} \times \cdots \times \mathfrak{C}_{m}} \mathfrak{P}\right|_{\eta\left(D_{1} \times \cdots \times D_{m}\right)} .
$$


Note that every $D_{j} \in \mathfrak{C}_{j}$ is a substructure of $\mathfrak{P}_{j}$, hence $\operatorname{rank}\left(D_{j}\right)<\omega^{\omega^{n}}$. Due to Lemma 3.17 .

$$
\left.\operatorname{rank}\left(\left.\mathfrak{P}\right|_{\eta\left(D_{1} \times \cdots \times D_{m}\right)}\right) \leq \operatorname{rank}\left(D_{1}\right) \otimes \cdots \otimes \operatorname{rank}\left(D_{m}\right)\right) \stackrel{(2.3)}{<} \omega^{\omega^{n}}
$$

Thus,

$$
\operatorname{rank}\left(\bigsqcup_{\left(D_{1}, \ldots, D_{m}\right) \in \mathfrak{C}_{1} \times \cdots \times \mathfrak{C}_{m}} \mathfrak{P} \uparrow_{\eta\left(D_{1} \times \cdots \times D_{m}\right)}\right) \leq \omega^{\omega^{n}} .
$$

Hence, the same holds for the substructure $\mathfrak{P} \uparrow_{\mu^{-1}(i)}$.

If $\mathfrak{P}$ is a forest, then without loss of generality $\mathfrak{P}_{2}, \ldots, \mathfrak{P}_{m}$ are disjoint unions of finite ordinals (cf. Remark 3.13). Thus, $\operatorname{rank}\left(\left.\mathfrak{P}\right|_{\eta\left(D_{1} \times \cdots \times D_{m}\right)}\right) \leq \operatorname{rank}\left(D_{1}\right) \otimes c=\alpha \otimes c$ for some finite ordinal $c<\omega$ and some $\alpha<\omega^{n}$. We get

$$
\alpha \otimes c \leq \underbrace{\alpha \oplus \alpha \oplus \cdots \oplus \alpha}_{c \text { many }} \stackrel{(2.2)}{<} \omega^{n}
$$

where the first inequality follows from the fact that every linearization of the direct product $\alpha \otimes c$ can be viewed as a linearization of the disjoint union of $c$ copies of $\alpha$ (one can actually show that equality holds at the place of the first inequality). We can now conclude completely analogously to the wulpo case.

We are now prepared to establish box-indecomposable intervals with respect to the rank function on the domain of wulpo's. Recall that we denote by rank $k_{u l}$ the function rank restricted to wulpo's and by rank $_{F}$ its restriction to well-founded forests.

Proposition 3.24. Let $i \in \mathbb{N}$.

(1) The interval $\left[\omega^{\omega^{i}}, \omega^{\omega^{i+1}}\right]$ is rank $_{u l}$-tamely-colorable-box-indecomposable.

(2) The interval $\left[\omega^{i}, \omega^{i+1}\right]$ is rank ${ }_{F}$-tamely-colorable-box-indecomposable.

Proof. For the first claim let $\mathfrak{P}=(P, \leq)$ be a wulpo of rank $\omega^{\omega^{i+1}}$. Heading for a contradiction, assume that it is a tamely colorable box-augmentation of wulpo's $\left(\mathfrak{P}_{1}, \ldots, \mathfrak{P}_{n}\right)$ such that $\operatorname{rank}\left(\mathfrak{P}_{j}\right)<\omega^{\omega^{i}}$ for all $1 \leq j \leq n$.

Take $c \in \mathbb{N}$ and the mapping $\mu: P \rightarrow\{1, \ldots, c\}$ from Lemma 3.21, The preimages $\mu^{-1}(i)$ of $\mu$ induce a sum-decomposition of $\mathfrak{P}$ into $c$ parts $\left.\mathfrak{P} \uparrow_{\mu^{-1}(1)}, \ldots, \mathfrak{P}\right\rceil_{\mu^{-1}(c)}$. Since $\omega^{\omega^{i+1}}$ is rank-sum-indecomposable by Proposition 3.10, we can assume (perhaps after renaming of colors) that $\operatorname{rank}\left(\left.\mathfrak{P}\right|_{\mu^{-1}(1)}\right)=\omega^{\omega^{i+1}}$. But Corollary 3.23 implies that $\operatorname{rank}\left(\mathfrak{P} \uparrow_{\mu^{-1}(1)}\right) \leq \omega^{\omega^{i}}$ which is clearly a contradiction.

For the case of forests, we use exactly the same arguments, and the second part of Corollary 3.23 yields a similar contradiction. ranks.

Using Propositions 3.9, 3.10 and 3.24, we obtain directly the desired bounds on the

Theorem 3.25.

(1) Every tree-automatic wulpo has rank strictly below $\omega^{\omega^{\omega}}$.

(2) Every tree-automatic well-founded forest has rank strictly below $\omega^{\omega}$. 
Proof. Concerning the first claim, let $\alpha_{i}=\omega^{\omega^{i}}$ for each $i \in \mathbb{N}$. Due to Propositions 3.10 and 3.24 (part (11)), the sequence $\alpha_{0}<\alpha_{1}<\alpha_{2}<\ldots$ satisfies the conditions of Proposition [3.9. For the sake of a contradiction to this latter proposition, assume that $\mathfrak{P}$ is a tree-automatic wulpo of rank at least $\omega^{\omega^{\omega}}$. Let $\varphi$ be the formula $\varphi(x, y)=x<y$. By wellfoundedness, for each $i \in \mathbb{N}$ there is a $p \in P$ such that $\left.\mathfrak{P}\right|_{\varphi, p}$ has rank $\alpha_{i}$, contradicting Proposition 3.9.

For (2), we proceed analogously with $\alpha_{i}=\omega^{i}$. This time we use the second part of Proposition 3.24.

Note that this result has an analogous counterpart in the world of string-automatic structures: Khoussainov and Minnes [16] and Delhommé [9] independently proved that the ordinal ranks of string-automatic well-founded partial orders are the ordinals strictly below $\omega^{\omega}$ and this bound is optimal because for each $\alpha<\omega^{\omega}$ the ordinal $\alpha$ is string-automatic. In contrast, we [14, 15] proved that every string-automatic forest has rank strictly below $\omega^{2}$ and this bound is also optimal. In the next section, we construct tree-automatic wellfounded trees of all ranks strictly below $\omega^{\omega}$. Moreover, since ordinals are wulpo's, our bound on the ranks of wulpo's is also optimal. Thus, tree-automatic forests realize much smaller ranks than tree-automatic partial orders (as it is also the case in the string-automatic setting). Note that there is no non-trivial bound on the ranks of arbitrary well-founded tree-automatic partial orders. But our new result on the subclass of all wulpo's and the analogy to the string-automatic case support the following conjecture.

Conjecture 3.26. Every tree-automatic well-founded partial order has rank below $\omega^{\omega^{\omega}}$.

Beside upwards linearity, well-founded forests also have the property that one cannot embed an infinite linear order. One might wonder whether the class of tree-automatic partial orders without infinite linear suborder already satisfies that the ranks are bounded by $\omega^{\omega}$. The example below answers this question negatively be showing that for each $\alpha<\omega^{\omega^{\omega}}$ there exists such a partial order of rank $\alpha$.

Example 3.27. Let $\alpha<\omega^{\omega^{\omega}}$ be an ordinal. We show that the the direct product of $\alpha$ and $\omega^{*}$ (the reverse of $\omega$ ) as strict partial orders is a tree-automatic partial order which has rank $\alpha$ and contains no infinite linear suborder.

More formally, we consider the partial order $\mathfrak{P}=(P, \preceq)$ on

$$
P=\{(\beta, k) \mid \beta<\alpha, k \in \mathbb{N}\}
$$

whose induced strict partial order $\prec$ is given by

$$
(\beta, k) \prec\left(\beta^{\prime}, k^{\prime}\right) \Longleftrightarrow \beta<\beta^{\prime} \text { and } k>k^{\prime} \text {. }
$$

As a direct product of the tree-automatic structures $\alpha$ and $\omega^{*}, \mathfrak{P}$ is also tree-automatic.

Note that $\mathfrak{P}$ does not contain an infinite linear suborder because any such suborder could be projected to an infinite strictly descending sequence in $\alpha$ or to an infinite strictly ascending sequence in $\omega^{*}$.

We prove that $\operatorname{rank}(\mathfrak{P})=\operatorname{rank}(\alpha)$ by transfinite induction. For $\beta=0$ and $k \in \omega^{*}$ we have $\operatorname{rank}((\beta, k), \mathfrak{P})=0$ because $(\beta, k)$ is a minimal element. For $\beta>0, k \in \omega^{*}$,

$$
\begin{aligned}
\operatorname{rank}((\beta, k), \mathfrak{P}) & =\sup \left\{\operatorname{rank}\left(\left(\beta^{\prime}, k^{\prime}\right), \mathfrak{P}\right)+1 \mid\left(\beta^{\prime}, k^{\prime}\right) \prec(\beta, k) \in P\right\} \\
& =\sup \left\{\beta^{\prime}+1 \mid \beta^{\prime}<\beta\right\}=\beta .
\end{aligned}
$$


Thus, we conclude that

$$
\operatorname{rank}(\mathfrak{P})=\sup \{\operatorname{rank}((\beta, k), \mathfrak{P})+1 \mid(\beta, k) \in \mathfrak{P}\}=\sup \{\beta+1 \mid \beta<\alpha\}=\alpha .
$$

The structure $\mathfrak{P}$ also shows that Delhommé's decomposition technique cannot be used to prove our conjecture that all tree-automatic partial orders have ranks below $\omega^{\omega^{\omega}}$. Note that for all $\alpha^{\prime} \in \alpha$ the substructure $\mathfrak{P} \uparrow_{\left\{\left(\alpha^{\prime}, n\right) \mid n \in \omega^{*}\right\}}$ is isomorphic to the countably infinite antichain $\left(\omega^{*},=\right)$. Similarly, for all $n \in \omega^{*}$ the substructure $\left.\mathfrak{P}\right|_{\left\{\left(\alpha^{\prime}, n\right) \mid \alpha^{\prime} \in \alpha\right\}}$ is isomorphic to the countably infinite antichain $(\alpha,=)$. Thus, $\mathfrak{P}$ is a partial order of rank $\alpha$ that is a tamely colorable box-augmentation of two antichains. Since infinite antichains have rank 1, we conclude that applications of box-decomposition to well-founded partial orders may destroy all information about the rank. Thus, there is no hope that one could provide bounds on the ranks of tree-automatic well-founded partial orders using Delhommé's decomposition technique.

\section{UPPER BOUND FOR THE ISOMORPHISM PROBLEM FOR WELL-FOUNDED TREES}

We first introduce hyperarithmetical sets. Afterwards we provide the upper bound for the isomorphism problem of well-founded tree-automatic trees.

4.1. Hyperarithmetical sets. We use standard terminology concerning recursion theory; see e.g. [24]. We use the definition of the hyperarithmetical hierarchy from Ash and Knight [1], see also [11. We first define inductively a set of ordinal notations $O \subseteq \mathbb{N}_{>0}$. Simultaneously we define a mapping $a \mapsto|a|_{O}$ from $O$ into ordinals and a strict partial order $<_{O}$ on $O$. The set $O$ is the smallest subset of $\mathbb{N}_{>0}$ satisfying the following conditions:

- $1 \in O$ and $|1|_{O}=0$, i.e., 1 is a notation for the ordinal 0 .

- If $a \in O$, then also $2^{a} \in O$. We set $\left|2^{a}\right|_{O}=|a|_{O}+1$ and let $b<_{O} 2^{a}$ if and only if $b=a$ or $b<_{O} a$.

- If $e \in \mathbb{N}$ is such that $\Phi_{e}$ (the $e^{t h}$ partial computable function) is total, $\Phi_{e}(n) \in O$ for all $n \in \mathbb{N}$, and $\Phi_{e}(0)<_{O} \Phi_{e}(1)<_{O} \Phi_{e}(2)<_{O} \cdots$, then also $3 \cdot 5^{e} \in O$. We set $\left|3 \cdot 5^{e}\right|_{O}=\sup \left\{\left|\Phi_{e}(n)\right|_{O} \mid n \in \mathbb{N}\right\}$ and let $b<_{O} 3 \cdot 5^{e}$ if and only if there exists $n \in \mathbb{N}$ with $b<_{O} \Phi_{e}(n)$.

An ordinal $\alpha$ is computable if there exists $a \in O$ with $|a|_{O}=\alpha$. The smallest non-computable ordinal is the Church-Kleene ordinal $\omega_{1}^{\mathrm{ck}}$. If $a \in O$ then the restriction of the partial order $\left(O,<_{O}\right)$ to $B=\left\{b \in O \mid b<_{O} a\right\}$ is isomorphic to the ordinal $|a|_{O}$ [1. Proposition 4.9]. Moreover, the set $B$ is computably enumerable and an index for $B$ can be computed from $a$ [1, Proposition 4.10].

Next, we define the hyperarithmetical hierarchy based on ordinal notations. For this we define sets $H(a)$ for each $a \in O$ as follows:

- $H(1)=\emptyset$,

- $H\left(2^{b}\right)=H(b)^{\prime}$ (the Turing jump of $H(b)$; see e.g. [24]),

- $H\left(3 \cdot 5^{e}\right)=\left\{\langle b, n\rangle \mid b<_{O} 3 \cdot 5^{e}, n \in H(b)\right\}$; here $\langle\cdot, \cdot\rangle$ denotes a computable pairing function.

Spector has shown that $|a|_{O}=|b|_{O}$ implies that $H(a)$ and $H(b)$ are Turing equivalent. The levels of the hyperarithmetical hierarchy can be defined as follows, where $\alpha$ is a computable ordinal. 
- If $\alpha$ is infinite, then $\Sigma_{\alpha}^{0}$ is the set of all subsets $A \subseteq \mathbb{N}$ that are recursively enumerable in some $H(a)$ with $|a|_{O}=\alpha$ (by Spector's theorem, the concrete choice of $a$ is irrelevant). For $n>0$ finite, one defines $\Sigma_{n}^{0}$ as the set of all subsets $A \subseteq \mathbb{N}$ that are recursively enumerable in $H(a)$ with $|a|_{O}=n-13$

- $\Pi_{\alpha}^{0}$ is the set of all complements of $\Sigma_{\alpha}^{0}$-sets.

- $\Delta_{\alpha}^{0}=\Sigma_{\alpha}^{0} \cap \Pi_{\alpha}^{0}$

A relation $R \subseteq \mathbb{N}^{k}$ is $X_{\alpha}^{0}$ (with $X \in\{\Sigma, \Pi, \Delta\}$ ) if the set $\left\{\left\langle x_{1}, \ldots, x_{k}\right\rangle \mid\left(x_{1}, \ldots, x_{k}\right) \in R\right\}$ is $X_{\alpha}^{0}$, where $\langle\cdot, \ldots, \cdot\rangle$ denotes a computable encoding of $k$-tuples.

For any two computable ordinals $\alpha, \beta, \alpha<\beta$ implies $\Sigma_{\alpha} \cup \Pi_{\alpha} \subsetneq \Delta_{\beta}$. The union of all classes $\Sigma_{\alpha}^{0}$ where $\alpha<\omega_{1}^{\text {ck }}$ yields the class of all hyperarithmetical sets. By a classical result of Kleene, the hyperarithmetical sets are exactly the sets in $\Delta_{1}^{1}=\Sigma_{1}^{1} \cap \Pi_{1}^{1}$, where $\Sigma_{1}^{1}$ is the first existential level of the analytical hierarchy, and $\Pi_{1}^{1}$ is the set of all complements of $\Sigma_{1}^{1}$-sets.

For our purposes, it is convenient to present an alternative definition of the hyperarithmetical hierarchy using computable infinitary formulas, see [1, Chapter 7]. Fix a predicate $R(\bar{x}) \subseteq \mathbb{N}^{k}$ where $k \geq 1$. If $R$ is computable, then a $\Sigma_{0}^{0}$ (resp. $\Pi_{0}^{0}$ ) index for $R$ is a triple $(\Sigma, 0, e)$ (resp. $(\Pi, 0, e))$ where $e$ is an index for $R$. Next, let $0<\alpha<\omega_{1}^{\text {ck }}$ be a computable ordinal. Case 1. $\alpha=\beta+1$ is a successor ordinal. Then, a $\Sigma_{\alpha}^{0}$ (resp. $\Pi_{\alpha}^{0}$ ) index for $R$ is a triple $(\Sigma, a, e)($ resp. $(\Pi, a, e))$ where $|a|_{O}=\alpha$ and $e$ is a $\Pi_{\beta}^{0}$ (resp. $\Sigma_{\beta}^{0}$ ) index for a predicate $P(\bar{x}, y) \subseteq \mathbb{N}^{k+1}$ such that for all $\bar{x} \in \mathbb{N}^{k}$ :

$$
R(\bar{x}) \Leftrightarrow \exists y: P(\bar{x}, y) \quad(\text { resp. } R(\bar{x}) \Leftrightarrow \forall y: P(\bar{x}, y)) .
$$

Case 2. $\alpha$ is a limit ordinal. Then, a $\Sigma_{\alpha}^{0}\left(\operatorname{resp} . \Pi_{\alpha}^{0}\right)$ index for $R$ is a triple $(\Sigma, a, e)$ (resp. $(\Pi, a, e))$, where $|a|_{O}=\alpha$ and $\Phi_{e}$ is a total computable function such that the following holds: For all $n \in \mathbb{N}, \Phi_{e}(n)$ is a $\Pi_{\beta_{n}}^{0}$ (resp. $\Sigma_{\beta_{n}}^{0}$ ) index for some predicate $P_{n}(\bar{x}) \subseteq \mathbb{N}^{k}$, $\beta_{0}<\beta_{1}<\ldots<\alpha$ with $\sup \left\{\beta_{n} \mid n \in \mathbb{N}\right\}=\alpha$, and for all $\bar{x} \in \mathbb{N}^{k}$ :

$$
R(\bar{x}) \Leftrightarrow \bigvee_{i \in \mathbb{N}} P_{i}(\bar{x}) \quad\left(\text { resp. } R(\bar{x}) \Leftrightarrow \bigwedge_{i \in \mathbb{N}} P_{i}(\bar{x})\right) .
$$

Essentially, we can view a $\Sigma_{\alpha}^{0}$ (resp. $\Pi_{\alpha}^{0}$ ) index as a finite representation of a computable infinitary formula that defines the corresponding $\Sigma_{\alpha}^{0}$ (resp. $\Pi_{\alpha}^{0}$ ) predicate. For instance, the $\Sigma_{\alpha}^{0}$ index $(\Sigma, a, e)$, where $|a|_{O}$ is a limit ordinal, represents the computable infinitary formula $\bigvee_{i \in \mathbb{N}} \varphi_{i}$, where $\varphi_{i}$ is the computable infinitary formula represented by the index $\Phi_{e}(i)$. In this paper we use the notions of $\Sigma_{\alpha}^{0}$ (resp. $\Pi_{\alpha}^{0}$ ) predicates and indices interchangeably. Formally, we do not allow negation in computable infinitary formulas. But if $\varphi(\bar{x})$ defines the $\Sigma_{\alpha}^{0}\left(\right.$ resp. $\left.\Pi_{\alpha}^{0}\right)$ set $A \subseteq \mathbb{N}^{k}$, then one can construct effectively a $\Pi_{\alpha}^{0}$ (resp. $\Sigma_{\alpha}^{0}$ ) formula for $\mathbb{N}^{k} \backslash A$; and we therefore may define this formula as $\neg \varphi(\bar{x})$, see [1, Theorem 7.1].

4.2. Hyperarithmetic Upper Bound. It turns out that the rank for well-founded computable trees yields an upper bound on the recursion-theoretic complexity of the isomorphism problem. Recall that we defined forests as particular partial orders. For the isomorphism problem, it is useful to assume that also the direct successor relation is computable. When speaking of a computable forest in the following theorem, we mean a forest $\mathfrak{F}=(F, \leq)$

\footnotetext{
${ }^{3}$ The distinction between finite and infinite ordinals is made in order to have a correspondence between the arithmetical hierarchy and the finite part of the hyperarithmetical hierarchy, see also [1, pp. 74 and 75].
} 
such that $F, \leq$, and the direct successor relation $E_{\mathfrak{F}}$ are all computable 4 Note that the direct successor relation of a tree-automatic forest is even tree-automatic because it is firstorder definable.

Theorem 4.1. Let $\alpha$ be a computable ordinal and assume that $\alpha=\lambda+k$, where $k \in \mathbb{N}$ and $\lambda$ is not a successor ordinal. The isomorphism problem for well-founded computable trees of rank at most $\alpha$ belongs to level $\Pi_{\lambda+2 k+1}^{0}$ of the hyperarithmetical hierarchy.

Proof. Let us fix a well-founded forest $\mathfrak{F}=(F, \leq)$. We define a computable infinitary $\Pi_{\lambda+2 k+1}^{0}$ formula expressing $\mathfrak{F}(x) \cong \mathfrak{F}(y)$ for nodes $x$ and $y$ of $\mathfrak{F}$ of rank at most $\lambda+k$, where $k \in \mathbb{N}$ and $\lambda=0$ or $\lambda$ is a limit ordinal. This suffices because the disjoint union of two computable trees is a computable forest.

Let $E=E_{\mathfrak{F}}$ be the direct successor relation of $\mathfrak{F}$. For every ordinal $\alpha$ we define a computable infinitary formula iso ${ }_{\alpha}(x, y)$ over $\mathfrak{F}$ as follows: Let

$$
\operatorname{iso}_{0}(x, y)=\forall z(\neg(x>z) \wedge \neg(y>z)) .
$$

This is a $\Pi_{1}^{0}$ formula. For a successor ordinal $\alpha+1$ let 5 iso $\alpha+1(x, y)$ be

$$
\forall u \in E(x) \cup E(y) \forall \ell \geq 1\left(\begin{array}{l}
\left.\exists^{\geq \ell} v \in E(x) \text { iso }_{\alpha}(u, v) \Longleftrightarrow \exists^{\geq \ell} v \in E(y) \text { iso }_{\alpha}(u, v)\right) \\
\wedge \operatorname{iso}_{\alpha}(u, u) .
\end{array}\right)
$$

Finally, for a limit ordinal $\alpha$, we define iso $\alpha(x, y)$ as

$$
\bigwedge_{\beta<\alpha} \forall u \in E(x) \cup E(y) \forall \ell \geq 1\left(\begin{array}{l}
\left.\exists^{\geq \ell} v \in E(x) \text { iso }_{\beta}(u, v) \Longleftrightarrow \exists^{\geq \ell} v \in E(y) \operatorname{iso}_{\beta}(u, v)\right) \\
\wedge \bigvee_{\beta<\alpha} \text { iso }_{\beta}(u, u) .
\end{array}\right)
$$

Let us argue by induction that iso ${ }_{\lambda+k}$ (where $k \in \mathbb{N}$ and $\lambda=0$ or $\lambda$ a limit ordinal) is a $\Pi_{\lambda+2 k+1}^{0}$-formula. The case $\lambda=k=0$ is clear. For the case $k=0$ and $\lambda>0$ note that the subformula $\forall u \in E(x) \cup E(y): \bigvee_{\beta<\lambda}$ iso $\beta(u, u)$ is $\Pi_{\lambda+1}^{0}$. Moreover, the subformula

$$
\forall u \in E(x) \cup E(y) \forall \ell \geq 1:\left(\exists^{\geq \ell} v \in E(x): \operatorname{iso}_{\beta}(u, v) \Longleftrightarrow \exists^{\geq \ell} v \in E(y): \operatorname{iso}_{\beta}(u, v)\right)
$$

is $\Pi_{\lambda^{\prime}+2 k^{\prime}+3}^{0}$, where $\beta=\lambda^{\prime}+k^{\prime}<\alpha$ (with $k^{\prime} \in \mathbb{N}$ and $\lambda^{\prime}=0$ or $\lambda^{\prime}$ a limit ordinal). Since $\lambda$ is a limit ordinal, we have $\lambda^{\prime}+2 k^{\prime}+3<\lambda$. This shows that iso $_{\lambda}(x, y)$ is a conjunction of a $\Pi_{\lambda}^{0}$-formula and a $\Pi_{\lambda+1}^{0}$-formula, and hence also $\Pi_{\lambda+1}^{0}$. Finally, for the case that

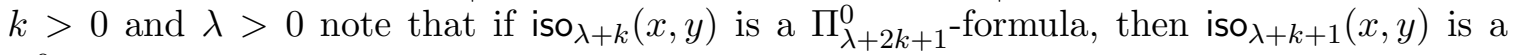
$\Pi_{\lambda+2 k+3}^{0}$-formula, since two alternating quantifier blocks are added for successor ordinals.

It remains to show that if $\operatorname{rank}(x) \leq \alpha$ and $\operatorname{rank}(y) \leq \alpha$, then $\mathfrak{F} \models$ iso $_{\alpha}(x, y)$ if and only if $\mathfrak{F}(x) \cong \mathfrak{F}(y)$. This is the content of Claim 2 below. As an auxiliary step we show the following claim.

Claim 1. If $\mathfrak{F} \models$ iso $_{\alpha}(x, y)$ then $\operatorname{rank}(x) \leq \alpha$ and $\operatorname{rank}(y) \leq \alpha$.

We prove Claim 1 by induction on $\alpha$. The case $\alpha=0$ is clear because trees of rank 0 do only consist of one node. Next, consider an ordinal $\alpha>0$ such that the claim holds for all $\beta<\alpha$. Assume that $\mathfrak{F} \models$ iso $_{\alpha}(x, y)$. Note that the second conjunct of

\footnotetext{
${ }^{4}$ On the other hand, if we would omit the requirement of a computable direct successor relation in Theorem 4.1 then we would only have to replace the constants in the theorem by larger values.

${ }^{5}$ We use $\exists^{\geq \ell} x: \varphi(x)$ as an abbreviation for $\exists x_{1} \cdots \exists x_{\ell}: \bigwedge_{1 \leq i<j \leq n} x_{i} \neq x_{j} \wedge \bigwedge_{i=1}^{n} \varphi\left(x_{i}\right)$. The quantifier $\exists^{\geq \ell} x$ can be encoded by an ordinary single existential quantifier.
} 
iso $_{\alpha}(x, y)$ and the induction hypothesis imply that all children of $x$ have rank $<\alpha$. Thus, $\operatorname{rank}(x) \leq \sup \{\beta+1 \mid \beta<\alpha\}=\alpha$. For $y$ we conclude analogously.

Claim 2. If $\operatorname{rank}(x) \leq \alpha$ and $\operatorname{rank}(y) \leq \alpha$, then $\mathfrak{F} \models$ iso $_{\alpha}(x, y)$ if and only if $\mathfrak{F}(x) \cong \mathfrak{F}(y)$.

We prove Claim 2 by induction on $\alpha$. Again, the case $\alpha=0$ is clear. Next, consider some ordinal $\alpha>0$ and assume that $\operatorname{rank}(x) \leq \alpha$ and $\operatorname{rank}(y) \leq \alpha$.

First, assume that $\mathfrak{F}(x) \cong \mathfrak{F}(y)$. Fix $\beta<\alpha, u \in E(x) \cup E(y)$, and $\ell \geq 1$. By assumption, we have $\operatorname{rank}(u)<\alpha$. Furthermore, due to Claim 1 , if $\beta<\operatorname{rank}(u)$, then there is no $v$ such that iso $_{\beta}(u, v)$ holds. Thus, in this case

$$
\left(\exists^{\geq \ell} v \in E(x): \operatorname{iso}_{\beta}(u, v) \Longleftrightarrow \exists^{\geq \ell} v \in E(y): \operatorname{iso}_{\beta}(u, v)\right) .
$$

holds. Moreover, if $\operatorname{rank}(u) \leq \beta<\alpha$, then Claim 1 and the induction hypothesis imply that

$$
\exists^{\geq \ell} v \in E(x): \operatorname{iso}_{\beta}(u, v) \Longleftrightarrow \exists^{\geq \ell} v \in E(y): \operatorname{iso}_{\beta}(u, v)
$$

is equivalent to

$$
\exists^{\geq \ell} v \in E(x): \mathfrak{F}(u) \cong \mathfrak{F}(v) \Longleftrightarrow \exists^{\geq \ell} v \in E(y): \mathfrak{F}(u) \cong \mathfrak{F}(v) .
$$

Since $\mathfrak{F}(x) \cong \mathfrak{F}(y)$ the latter is clearly satisfied. Moreover, by induction hypothesis for each $u \in E(x) \cup E(y)$ we have $\operatorname{iso}_{\operatorname{rank}(u)}(u, u)$ (note that $\operatorname{rank}(u)<\alpha$ ). Hence $\bigvee_{\beta<\alpha}$ iso $_{\beta}(u, u)$ holds as well. Thus iso $\alpha(x, y)$ is satisfied.

For the other direction, assume that $\mathfrak{F} \models$ iso $_{\alpha}(x, y)$. Then for each $u \in E(x) \cup E(y)$, there is some $\beta<\alpha$ such that iso $\beta(u, u)$ holds. Due to Claim 1 this implies that $\operatorname{rank}(u)<\alpha$ for each child $u$ of $x$ or $y$. Using the induction hypothesis and Claim 1, we conclude that for all $u \in E(x) \cup E(y)$ and all ordinals $\operatorname{rank}(u) \leq \beta<\alpha$,

$$
\exists^{\geq \ell} v \in E(x): \operatorname{iso}_{\beta}(u, v) \Longleftrightarrow \exists^{\geq \ell} v \in E(y): \operatorname{iso}_{\beta}(u, v)
$$

is equivalent to

$$
\exists^{\geq \ell} v \in E(x): \mathfrak{F}(u) \cong \mathfrak{F}(v) \Longleftrightarrow \exists^{\geq \ell} v \in E(y): \mathfrak{F}(u) \cong \mathfrak{F}(v) .
$$

Since iso $\alpha(x, y)$ holds, for each $u \in E(x) \cup E(y)$ there is a $\operatorname{rank}(u) \leq \beta<\alpha$ such that (4.1) holds. Hence, (4.2) holds for all children $u$ of $x$ or $y$, which implies that $\mathfrak{F}(x) \cong \mathfrak{F}(y)$.

Remark 4.2. If we are only interested in the isomorphism problem for computable trees of rank at most $\lambda$ for a limit ordinal $\lambda$ it suffices to consider the $\Pi_{\lambda}^{0}$-formula

$$
\bigwedge_{\beta<\alpha} \forall u \in E(x) \cup E(y) \forall \ell \geq 1:\left(\exists^{\geq \ell} v \in E(x): \operatorname{iso}_{\beta}(u, v) \Longleftrightarrow \exists^{\geq \ell} v \in E(y): \operatorname{iso}_{\beta}(u, v)\right)
$$

(where the formulas iso $\beta$ are constructed as above). This is because if the rank of the root is bounded by $\lambda$, any child of the root has rank strictly below $\lambda$ and satisfies $\operatorname{iso}_{\beta}(u, u)$ for some $\beta<\lambda$.

Corollary 4.3. The isomorphism problem for well-founded tree-automatic trees belongs to $\Delta_{\omega \omega}^{0}$.

Proof. The $\Sigma_{\omega^{\omega}}^{0}$ formula $\bigvee_{\alpha<\omega^{\omega}}$ iso $_{\alpha}(x, y)$ expresses $\mathfrak{F}(x) \cong \mathfrak{F}(y)$ for all $x, y$ with $\operatorname{rank}(x)$, $\operatorname{rank}(y)<\omega^{\omega}$.

With Remark 4.2 , we also obtain a $\Pi_{\omega^{\omega}}^{0}$ formula expressing $\mathfrak{F}(x) \cong \mathfrak{F}(y)$ for all $x, y$ with $\operatorname{rank}(x), \operatorname{rank}(y)<\omega^{\omega}$.

Due to Theorem 3.25, the rank of every tree-automatic well-founded tree is strictly below $\omega^{\omega}$. Thus, the isomorphism problem for these trees belongs to $\Sigma_{\omega^{\omega}}^{0} \cap \Pi_{\omega^{\omega}}^{0}=\Delta_{\omega^{\omega}}^{0}$. 


\section{LOWER BOUND FOR THE ISOMORPHISM PROBLEM FOR WELL-FOUNDED TREES}

In this section, we prove hyperarithmetical lower bounds for the isomorphism problem for well-founded tree-automatic trees. More precisely, we show that for every ordinal $\omega^{i}$, there exists a well-founded tree $\mathfrak{V}$ such that the set of all tree-automatic copies of $\mathfrak{V}$ is hard for the class $\Pi_{\omega^{i}}^{0}$. Moreover, for the class of all well-founded tree-automatic trees, we prove that the isomorphism problem is $\Delta_{\omega^{\omega}}^{0}$-hard under Turing-reductions.

5.1. Isomorphism for computable trees of rank $<\omega^{\omega}$. Basically, our hardness proof is a reduction from computable well-founded trees to tree-automatic well-founded trees. For this, we make use of a construction from [11, which works for all computable ordinals. We use this construction only for ordinals strictly below $\omega^{\omega}$. In this section, a computable tree is a computable prefix-closed subset $\mathfrak{S} \subseteq \mathbb{N}_{>0}^{*} 6$ We identify $\mathfrak{S}$ with the tree $(\mathfrak{S}, \succeq)$. Recall that $\succeq$ is the inverse prefix relation. Hence, the empty word $\varepsilon$ is the root of $(\mathfrak{S}, \succeq)$.

First, we have to fix the so called fundamental sequence for every limit ordinal $<\omega^{\omega}$. Each ordinal $\alpha<\omega^{\omega}$ can be written in its Cantor normal form as

$$
\alpha=\omega^{e_{i}} \cdot n_{i}+\omega^{e_{i-1}} \cdot n_{i-1}+\cdots+\omega^{e_{1}} \cdot n_{1},
$$

where $e_{i}>e_{i-1}>\cdots>e_{1} \geq 0$ and $n_{j}>0$ are natural numbers for $1 \leq j \leq i$. Assume that $e_{1}>0$ so that $\alpha$ is a limit ordinal. Then

$$
\alpha=\sup \left\{\alpha_{k} \mid k \geq 1\right\},
$$

where we define $\alpha_{k}$ as

$$
\alpha_{k}=\omega^{e_{i}} \cdot n_{i}+\omega^{e_{i-1}} \cdot n_{i-1}+\cdots+\omega^{e_{2}} \cdot n_{2}+\omega^{e_{1}} \cdot\left(n_{1}-1\right)+\omega^{e_{1}-1} \cdot k+1,
$$

for $k \geq 1$. Note that $\alpha_{k}$ is a successor ordinal and we call $\left(\alpha_{k}\right)_{k \geq 1}$ the fundamental sequence.

Next, we define for every ordinal $\alpha<\omega^{\omega}$ a computable well-founded tree $\mathfrak{S}_{\alpha} \subseteq \mathbb{N}_{>0}^{*}$ by induction on $\alpha$. Let $\mathfrak{S}_{0}=\{\varepsilon\}$ be the tree consisting of a single node. If $\alpha=\beta+1<\omega^{\omega}$ is a successor ordinal then

$$
\mathfrak{S}_{\beta+1}=\left\{n u \mid n \in \mathbb{N}_{>0}, u \in \mathfrak{S}_{\beta}\right\} \cup\{\varepsilon\} .
$$

Hence, $\mathfrak{S}_{\beta+1}$ consists of $\aleph_{0}$ many copies of $\mathfrak{S}_{\beta}$ together with a new root. Finally let $\alpha<\omega^{\omega}$ be a limit ordinal with the fundamental sequence $\left(\alpha_{k}\right)_{k \geq 1}$ defined in (5.1). Then

$$
\mathfrak{S}_{\alpha}=\left\{k u \mid k \in \mathbb{N}_{>0}, u \in \mathfrak{S}_{\alpha_{k}}\right\} \cup\{\varepsilon\} .
$$

Thus, $\mathfrak{S}_{\alpha}$ consists of all the trees $\mathfrak{S}_{\alpha_{k}}\left(k \in \mathbb{N}_{>0}\right)$ together with a new root. By induction on $\alpha<\omega^{\omega}$, it is straightforward to show that $\mathfrak{S}_{\alpha}$ is well-founded and computable. Moreover, also the set of leaves leaves $\left(\mathfrak{S}_{\alpha}\right) \subseteq \mathbb{N}_{>0}^{*}$ is computable. Let $A \subseteq$ leaves $\left(\mathfrak{S}_{\alpha}\right)$. Then we denote the structure consisting of the tree $\mathfrak{S}_{\alpha}$ together with the additional unary predicate $A$ by $\left(\mathfrak{S}_{\alpha}, A\right)$.

The following result is implicitly shown in [11, Proposition 3.2], where it is stated for all computable ordinals. But since we only defined fixed fundamental sequences for limit ordinals below $\omega^{\omega}$, we restrict to ordinals below $\omega^{\omega}$.

\footnotetext{
${ }^{6}$ For technical reasons, it is useful to exclude 0. This makes the construction of tree-automatic copies easier.
} 
Theorem 5.1. Given a limit ordinal $\alpha<\omega^{\omega}$ and $k \in \mathbb{N}_{>0} \cup\{\infty\}$ (resp., a successor ordinal $\left.\alpha<\omega^{\omega}\right)$, one can compute indices of a computable subset $L_{k}^{\alpha} \subseteq$ leaves $\left(\mathfrak{S}_{\alpha}\right.$ ) (resp., computable subsets $A_{\alpha}, E_{\alpha} \subseteq$ leaves $\left(\mathfrak{S}_{\alpha}\right)$ ) such that the following holds:

From a natural number $n \in \mathbb{N}$ and $a \Pi_{\alpha}^{0}$ index $(\Pi, a, e)$ for a set $P \subseteq \mathbb{N}$ one can compute an index for a computable subset $T_{P, n} \subseteq$ leaves $\left(\mathfrak{S}_{\alpha}\right)$ such that the following holds:

- $\left(\mathfrak{S}_{\alpha}, A_{\alpha}\right) \neq\left(\mathfrak{S}_{\alpha}, E_{\alpha}\right)$ and $\left(\mathfrak{S}_{\alpha}, L_{\infty}^{\alpha}\right) \neq\left(\mathfrak{S}_{\alpha}, L_{k}^{\alpha}\right)$ for all $k \in \mathbb{N}_{>0}$, respectively.

- If $\alpha$ is a successor ordinal, then

$$
\left(\mathfrak{S}_{\alpha}, T_{P, n}\right) \cong \begin{cases}\left(\mathfrak{S}_{\alpha}, A_{\alpha}\right) & \text { if } n \in P \\ \left(\mathfrak{S}_{\alpha}, E_{\alpha}\right) & \text { if } n \notin P .\end{cases}
$$

- If $\alpha$ is a limit ordinal, then

$$
\left(\mathfrak{S}_{\alpha}, T_{P, n}\right) \cong \begin{cases}\left(\mathfrak{S}_{\alpha}, L_{\infty}^{\alpha}\right) & \text { if } n \in P \\ \left(\mathfrak{S}_{\alpha}, L_{k}^{\alpha}\right) \text { for some } k \in \mathbb{N}_{>0} & \text { if } n \notin P .\end{cases}
$$

Proof. In Proposition 3.2 from [11], the computable subset $A_{\alpha} \subseteq$ leaves $\left(\mathfrak{S}_{\alpha}\right)$ is replaced by a computable tree $\mathcal{A}_{\alpha} \subseteq \mathfrak{S}_{\alpha}$ (and similarly for $\left.E_{\alpha}, L_{k}^{\alpha}, T_{P, n}\right)$. For our purpose it is more convenient to work with computable subsets of the leaves of the fixed tree $\mathfrak{S}_{\alpha}$. Nevertheless, the construction works analogously to the proof of Proposition 3.2 from [11, except for the induction base $\alpha=1$. In [11], the tree $\mathcal{A}_{1}$ consists of a single node and the tree $\mathcal{E}_{1}$ consists of a root with infinitely many children (i.e., $\mathcal{E}_{1} \cong \mathfrak{S}_{1}$ ). For the construction of $T_{P, n}$, one chooses a computable set $Q \subseteq \mathbb{N} \times \mathbb{N}$ such that $P^{\prime}=\{n \mid \exists x Q(x, n)\}$ is the complement of the $\Pi_{1}^{0}$-set $P$. Then the tree $\mathcal{T}_{P, n}$ consists of the root and $x \in \mathbb{N}_{>0}$ is a child of the root if and only if there exists $y \leq x$ satisfying $Q(y, n)$. Hence, $\mathcal{T}_{P, n} \cong \mathcal{E}_{1}$ if there exists $x$ with $Q(x, n)$ and $\mathcal{T}_{P, n} \cong \mathcal{A}_{1}$ if $\neg Q(x, n)$ for all $x$.

In our context, we define the subsets $A_{1}, E_{1}, T_{P, n} \subseteq \mathbb{N}_{>0}=$ leaves $\left(\mathfrak{S}_{1}\right)$ as follows: let $A_{1}=\emptyset, E_{1}$ be the set of all non-zero even numbers, and $T_{P, n}=\{2 x \mid \exists y \leq x: Q(y, n)\}$. Then, we have $\left(\mathfrak{S}_{1}, T_{P, n}\right) \cong\left(\mathfrak{S}_{1}, E_{1}\right)$ if there exists $x$ with $Q(x, n)$ and $\left(\mathfrak{S}_{1}, T_{P, n}\right) \cong\left(\mathfrak{S}_{1}, A_{1}\right)$ if $\neg Q(x, n)$ for all $x$.

Let us now discuss the induction step. As remarked above, it works analogously to the proof of Proposition 3.2 from [11, but we present the details for the sake of completeness. Let $\alpha<\omega^{\omega}, n \in \mathbb{N}$, and $(\Pi, a, e)$ be a $\Pi_{\alpha}^{0}$ index for a set $P \subseteq \mathbb{N}$. Hence, $|a|_{O}=\alpha$. We distinguish three cases:

Case 1. $\alpha=\beta+1$ for a successor ordinal $\beta<\omega^{\omega}$. Note that we can compute indices for the sets $A_{\beta}, E_{\beta} \subseteq$ leaves $\left(\mathfrak{S}_{\beta}\right)$ from $\beta$. The following constructions are illustrated in Figure 2. We define the sets $A_{\alpha}, E_{\alpha} \subseteq$ leaves $\left(\mathfrak{S}_{\alpha}\right)=\left\{m u \mid m \in \mathbb{N}_{>0}, u \in \operatorname{leaves}\left(\mathfrak{S}_{\beta}\right)\right\}$ as follows (here and in the following, we use square brackets to enclose single numbers in finite words over $\mathbb{N}$ ):

$$
\begin{aligned}
& A_{\alpha}=\left\{m u \mid n \in \mathbb{N}_{>0}, u \in E_{\beta}\right\}, \\
& E_{\alpha}=\left\{[2 m] u \mid n \in \mathbb{N}_{>0}, u \in E_{\beta}\right\} \cup\left\{[2 m-1] u \mid m \in \mathbb{N}_{>0}, u \in A_{\beta}\right\} .
\end{aligned}
$$

Clearly, indices for these computable sets can be computed from the indices for $A_{\beta}$ and $E_{\beta}$. Since $\left(\mathfrak{S}_{\beta}, E_{\beta}\right) \neq\left(\mathfrak{S}_{\beta}, A_{\beta}\right)$, we also have $\left(\mathfrak{S}_{\alpha}, E_{\alpha}\right) \neq\left(\mathfrak{S}_{\alpha}, A_{\alpha}\right)$

The index $e$ from $(\Pi, a, e)$ is a $\Sigma_{\beta}^{0}$ index for a set $Q \subseteq \mathbb{N} \times \mathbb{N}$ such that $n \in P$ if and only if $(n, m) \in Q$ for all $m \in \mathbb{N}$. From $e$ one can compute a $\Pi_{\beta}^{0}$ index $f$ for the complement of $Q$ [1, Theorem 7.1]. By induction, one can compute from $f$ and $(n, m) \in \mathbb{N} \times \mathbb{N}$ an index 

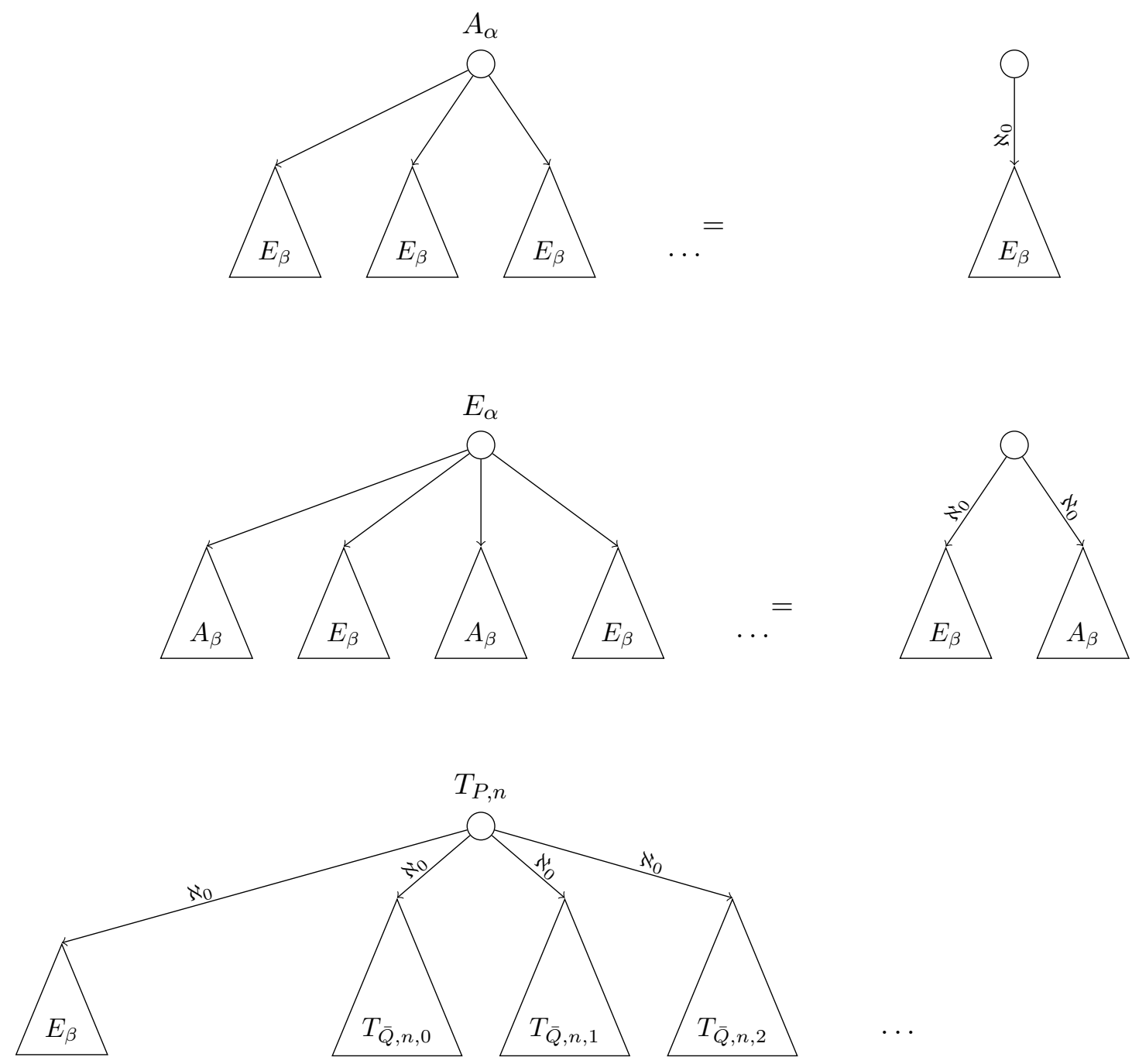

Figure 2: Trees for Case 1 from the proof of Theorem 5.1 (here and in the following pictures we write an $\aleph_{0}$ labeled edge from a node $d$ to a subtree $T$ if $d$ has edges to countably infinitely many subtrees isomorphic to $T$ ).

of a computable set $T_{\bar{Q}, n, m} \subseteq$ leaves $\left(\mathfrak{S}_{\beta}\right)$ such that: 7

$$
\left(\mathfrak{S}_{\beta}, T_{\bar{Q}, n, m}\right) \cong \begin{cases}\left(\mathfrak{S}_{\beta}, A_{\beta}\right) & \text { if }(n, m) \notin Q, \\ \left(\mathfrak{S}_{\beta}, E_{\beta}\right) & \text { if }(n, m) \in Q .\end{cases}
$$

We define the computable set $T_{P, n} \subseteq$ leaves $\left(\mathfrak{S}_{\alpha}\right)$ as follows (where we write $2^{\mathbb{N}} 3^{\mathbb{N}}$ for $\left.\left\{n \in \mathbb{N} \mid \exists k, l \in \mathbb{N} n=2^{k} \cdot 3^{l}\right\}\right)$ :

$$
T_{P, n}=\left\{\left[2^{m} 3^{x}\right] u \mid x, m \in \mathbb{N}, u \in T_{\bar{Q}, n, m}\right\} \cup\left\{x u \mid x \in \mathbb{N}_{>0} \backslash 2^{\mathbb{N}} 3^{\mathbb{N}}, u \in E_{\beta}\right\} .
$$

\footnotetext{
${ }^{7}$ Formally, we have to encode the pair $(n, m)$ into a single number in order to apply the induction hypothesis.
} 

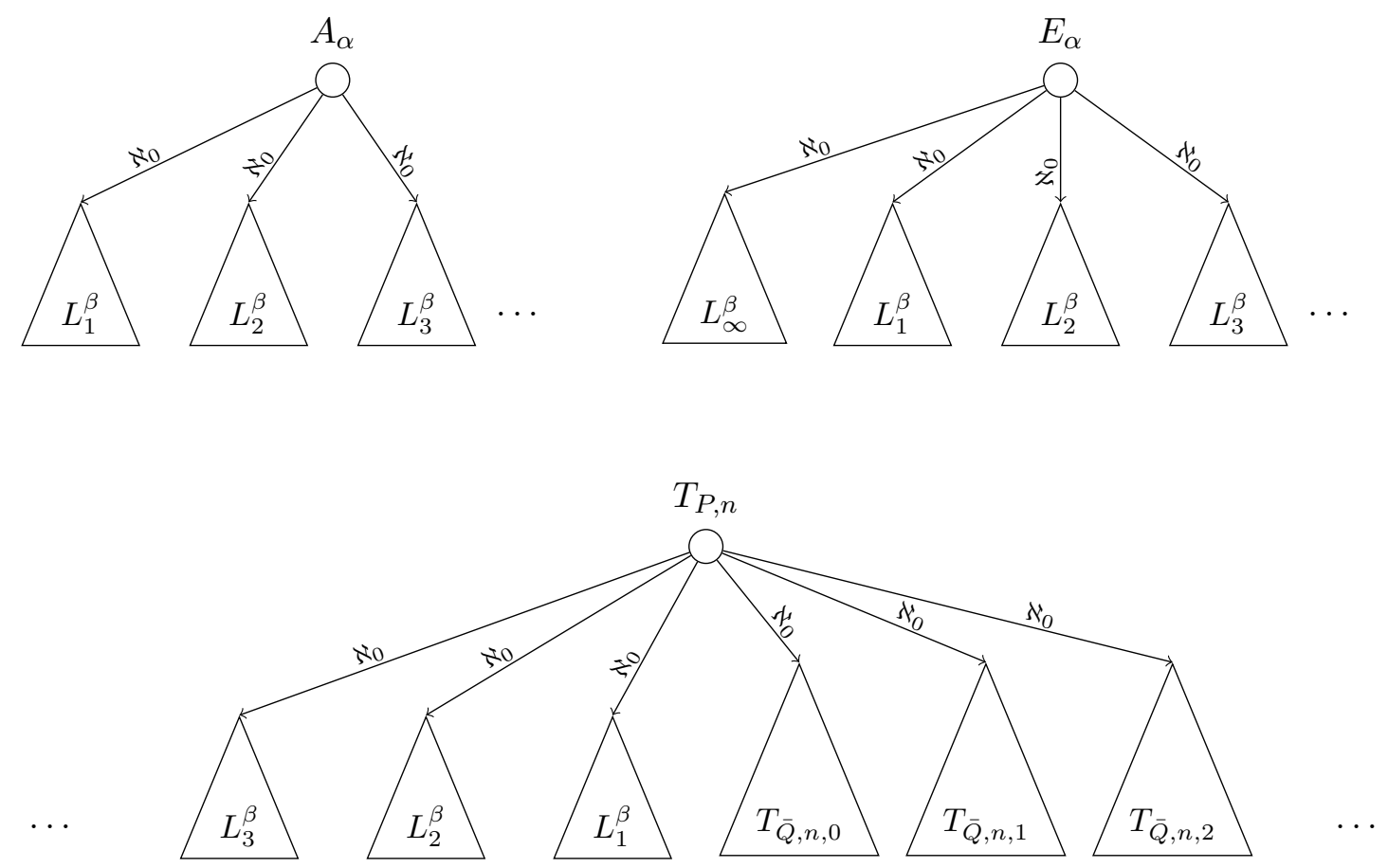

Figure 3: Trees for Case 2 from the proof of Theorem 5.1,

Note that the leaf-labeled tree $\left(\mathfrak{S}_{\alpha}, T_{P, n}\right)$ consists of a root together with infinitely many copies of each of the trees $\left(\mathfrak{S}_{\beta}, T_{\bar{Q}, n, m}\right)$ for $m \in \mathbb{N}$ together with infinitely many copies of the tree $\left(\mathfrak{S}_{\beta}, E_{\beta}\right)$. Also note that each of the subtrees $\left(\mathfrak{S}_{\beta}, T_{\bar{Q}, n, m}\right)$ is either isomorphic to $\left(\mathfrak{S}_{\beta}, A_{\beta}\right)$ or $\left(\mathfrak{S}_{\beta}, E_{\beta}\right)$.

If there is an $m$ with $(n, m) \notin Q$, then $\left(\mathfrak{S}_{\alpha}, T_{P, n}\right)$ consists of infinitely many copies of $\left(\mathfrak{S}_{\beta}, A_{\beta}\right)$ and infinitely many copies of $\left(\mathfrak{S}_{\beta}, E_{\beta}\right)$. Hence, we have $\left(\mathfrak{S}_{\alpha}, T_{P, n}\right) \cong\left(\mathfrak{S}_{\alpha}, E_{\alpha}\right)$. On the other hand, if $(n, m) \in Q$ for all $m \in \mathbb{N}$ then $\left(\mathfrak{S}_{\alpha}, T_{P, n}\right) \cong\left(\mathfrak{S}_{\alpha}, A_{\alpha}\right)$.

Finally, an index for the computable set $T_{P, n}$ can be computed from $(\Pi, a, e)$ and $n$.

Case 2. $\alpha=\beta+1$ for a limit ordinal $\beta<\omega^{\omega}$. Hence, from a given $k \in \mathbb{N}_{>0} \cup\{\infty\}$ one can compute and index of a computable subset $L_{k}^{\alpha} \subseteq$ leaves $\left(\mathfrak{S}_{\beta}\right)$. We define the sets $A_{\alpha}, E_{\alpha} \subseteq$ leaves $\left(\mathfrak{S}_{\alpha}\right)=\left\{m u \mid m \in \mathbb{N}_{>0}, u \in\right.$ leaves $\left.\left(\mathfrak{S}_{\beta}\right)\right\}$ as follows (cf. Figure 3):

$$
\begin{aligned}
& A_{\alpha}=\left\{\left[2^{k-1}(2 x-1)\right] u \mid k, x \in \mathbb{N}_{>0}, u \in L_{k}^{\beta}\right\} \\
& E_{\alpha}=\left\{\left[2^{k}(2 x-1)\right] u \mid k, x \in \mathbb{N}_{>0}, u \in L_{k}^{\beta}\right\} \cup\left\{[2 x-1] u \mid x \in \mathbb{N}_{>0}, u \in L_{\infty}^{\beta}\right\} .
\end{aligned}
$$

Again, indices for these computable sets can be computed from $\alpha$. Since $\left(\mathfrak{S}_{\beta}, L_{k}^{\beta}\right) \nsucceq$ $\left(\mathfrak{S}_{\beta}, L_{\infty}^{\beta}\right)$ for all $k \in \mathbb{N}_{>0}$, we have $\left(\mathfrak{S}_{\alpha}, E_{\alpha}\right) \neq\left(\mathfrak{S}_{\alpha}, A_{\alpha}\right)$.

As in Case 1, the index $e$ from $(\Pi, a, e)$ is a $\Sigma_{\beta}^{0}$ index for a set $Q \subseteq \mathbb{N} \times \mathbb{N}$ such that $n \in P$ if and only if $(n, m) \in Q$ for all $m \in \mathbb{N}$. From $e$ one can compute a $\Pi_{\beta}^{0}$ index $f$ for the complement of $Q$. By induction, one can compute from $f$ and $(n, m) \in \mathbb{N} \times \mathbb{N}$ an index 
of a computable set $T_{\bar{Q}, n, m} \subseteq$ leaves $\left(\mathfrak{S}_{\beta}\right)$ such that

$$
\left(\mathfrak{S}_{\beta}, T_{\bar{Q}, n, m}\right) \cong \begin{cases}\left(\mathfrak{S}_{\beta}, L_{\infty}^{\beta}\right) & \text { if }(n, m) \notin Q, \\ \left(\mathfrak{S}_{\beta}, L_{k}^{\beta}\right) \text { for some } k \in \mathbb{N}_{>0} & \text { if }(n, m) \in Q .\end{cases}
$$

We define the computable set $T_{P, n} \subseteq$ leaves $\left(\mathfrak{S}_{\alpha}\right)$ as the set

$$
\left\{\left[2^{2 m}(2 x+1)\right] u \mid m, x \in \mathbb{N}, u \in T_{\bar{Q}, n, m}\right\} \cup\left\{\left[2^{2 k-1}(2 x+1)\right] u \mid k \in \mathbb{N}_{>0}, x \in \mathbb{N}, u \in L_{k}^{\beta}\right\} .
$$

The leaf-labeled tree $\left(\mathfrak{S}_{\alpha}, T_{P, n}\right)$ consists of a root together with infinitely many copies of each of the trees $\left(\mathfrak{S}_{\beta}, T_{\bar{Q}, n, m}\right)$ for $m \in \mathbb{N}$ together with infinitely many copies of each of the trees $\left(\mathfrak{S}_{\beta}, L_{k}^{\beta}\right)$ for $k \in \mathbb{N}_{>0}$. Also note that each of the subtrees $\left(\mathfrak{S}_{\beta}, T_{\bar{Q}, n, m}\right)$ is isomorphic to one of the trees $\left(\mathfrak{S}_{\beta}, L_{k}^{\beta}\right)\left(k \in \mathbb{N}_{>0} \cup\{\infty\}\right)$.

If there is an $m$ with $(n, m) \notin Q$, then $\left(\mathfrak{S}_{\alpha}, T_{P, n}\right)$ consists of infinitely many copies of each of the trees $\left(\mathfrak{S}_{\beta}, L_{k}^{\beta}\right)$ for all $k \in \mathbb{N}_{>0} \cup\{\infty\}$. Hence, we have $\left(\mathfrak{S}_{\alpha}, T_{P, n}\right) \cong\left(\mathfrak{S}_{\alpha}, E_{\alpha}\right)$. On the other hand, if $(n, m) \in Q$ for all $m \in \mathbb{N}$ then $\left(\mathfrak{S}_{\alpha}, T_{P, n}\right) \cong\left(\mathfrak{S}_{\alpha}, A_{\alpha}\right)$.

Case 3. $\alpha$ is a limit ordinal. Let $\left(\alpha_{k}\right)_{k \geq 1}$ be the fundamental sequence of $\alpha$. Recall that $\alpha_{k}$ is a successor ordinal. We define a computable subset $L_{\infty}^{\alpha}$ of the leaves leaves $\left(\mathfrak{S}_{\alpha}\right)=$ $\left\{k u \mid k \in \mathbb{N}_{>0}, u \in\right.$ leaves $\left.\left(\mathfrak{S}_{\alpha_{k}}\right)\right\}$ by

$$
L_{\infty}^{\alpha}=\left\{k u \mid k \in \mathbb{N}_{>0}, u \in A_{\alpha_{k}}\right\} .
$$

For $k \in \mathbb{N}_{>0}$ we define the computable set $L_{k}^{\alpha} \subseteq$ leaves $\left(\mathfrak{S}_{\alpha}\right)$ by

$$
L_{k}^{\alpha}=\left\{x u \mid 0<x<k, u \in A_{\alpha_{x}}\right\} \cup\left\{x u \mid x \geq k, u \in E_{\alpha_{x}}\right\} .
$$

Recall that $\left(\mathfrak{S}_{\alpha_{k}}, A_{\alpha_{k}}\right) \varsubsetneqq\left(\mathfrak{S}_{\alpha_{k}}, E_{\alpha_{k}}\right)$ for all $k \geq 1$, whence $\left(\mathfrak{S}_{\alpha}, L_{k}^{\alpha}\right) \varsubsetneqq\left(\mathfrak{S}_{\alpha}, L_{\infty}^{\alpha}\right)$ for all $k \geq 1$.

For the $\Pi_{\alpha}^{0}$ index $(\Pi, a, e)$ we can assume (using a padding argument) that $\Phi_{e}$ is a total computable function such that for all $k \geq 1, \Phi_{e}(k)$ is a $\Pi_{\alpha_{k}}^{0}$ index for some set $Q_{k} \subseteq \mathbb{N}$ and $P=\bigcap_{k>1} Q_{k}$. Let us define for $k \in \mathbb{N}_{>0}$ the set $P_{k}=\bigcap_{i=1}^{k} Q_{k}$. Since $\Pi_{\alpha_{k}}^{0}$ sets are closed under finite intersections, $P_{k}$ is a $\Pi_{\alpha_{k}}^{0}$ set. Moreover, a $\Pi_{\alpha_{k}}^{0}$ index for $P_{k}$ can be computed from $k$. By induction, from $k \in \mathbb{N}_{>0}$ and $n$ one can compute an index for a computable set $T_{P_{k}, n} \subseteq$ leaves $\left(\mathfrak{S}_{\alpha_{k}}\right)$ such that

$$
\left(\mathfrak{S}_{\alpha_{k}}, T_{P_{k}, n}\right) \cong \begin{cases}\left(\mathfrak{S}_{\alpha_{k}}, A_{\alpha_{k}}\right) & \text { if } n \in P_{k}, \\ \left(\mathfrak{S}_{\alpha_{k}}, E_{\alpha_{k}}\right) & \text { if } n \notin P_{k} .\end{cases}
$$

Define the computable set

$$
T_{P, n}=\left\{k u \mid k \in \mathbb{N}_{>0}, u \in T_{P_{k}, n}\right\} .
$$

If $n \in P$, then $n \in P_{k}$ for all $k \in \mathbb{N}_{>0}$ and we get $\left(\mathfrak{S}_{\alpha}, T_{P, n}\right) \cong\left(\mathfrak{S}_{\alpha}, L_{\infty}^{\alpha}\right)$. On the other hand, if $n \notin P$ then there is some $k \in \mathbb{N}_{>0}$ such that $n \in P_{i}$ if and only if $i<k$. In this case we get $\left(\mathfrak{S}_{\alpha}, T_{P, n}\right) \cong\left(\mathfrak{S}_{\alpha}, L_{k}^{\alpha}\right)$. 

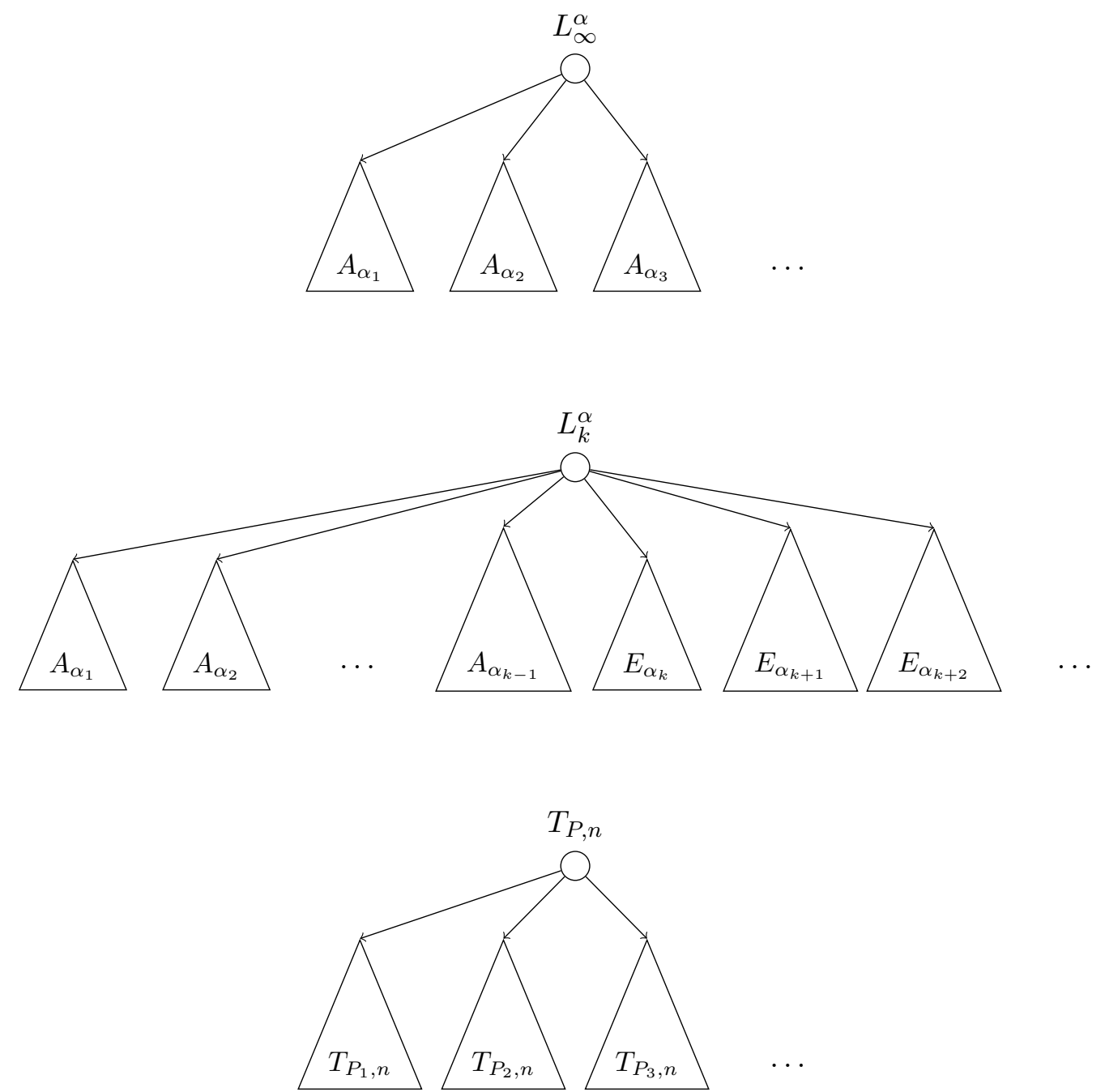

Figure 4: Trees for Case 3 from the proof of Theorem 5.1.

5.2. Tree-automaticity of the trees $\mathfrak{S}_{\omega^{i}}$. In this section, we show that all trees $\mathfrak{S}_{\omega^{i}}$ $(i \geq 1)$ from the previous section are tree-automatic. For this, we need the following lemma.

Lemma 5.2. Let $i \geq 1, n \geq 1, \alpha \leq \omega^{i}$. Then we have

$$
\mathfrak{S}_{\alpha} \cup\left\{u v \mid u \in \operatorname{leaves}\left(\mathfrak{S}_{\alpha}\right), v \in \mathfrak{S}_{\omega^{i} \cdot n}\right\}=\mathfrak{S}_{\omega^{i} \cdot n+\alpha} .
$$

Note that the tree on the left-hand side of (5.2) is the tree that results from the tree $\mathfrak{S}_{\alpha}$ by replacing every leaf by a copy of the tree $\mathfrak{S}_{\omega^{i} \cdot n}$.

Proof. We prove the lemma by induction on $\alpha \leq \omega^{i}$. The case $\alpha=0$ is clear. Next, assume that $\alpha=\gamma+1$ is a successor ordinal. Then

$$
\mathfrak{S}_{\alpha}=\{\varepsilon\} \cup\left\{n u \mid n \in \mathbb{N}_{>0}, u \in \mathfrak{S}_{\gamma}\right\} .
$$


By induction hypothesis, we have

$$
\mathfrak{S}_{\gamma} \cup\left\{u v \mid u \in \operatorname{leaves}\left(\mathfrak{S}_{\gamma}\right), v \in \mathfrak{S}_{\omega^{i} \cdot n}\right\}=\mathfrak{S}_{\omega^{i} \cdot n+\gamma} .
$$

Hence, we get

$$
\begin{aligned}
& \mathfrak{S}_{\alpha} \cup\left\{u v \mid u \in \operatorname{leaves}\left(\mathfrak{S}_{\alpha}\right), v \in \mathfrak{S}_{\omega^{i} \cdot n}\right\} \\
& \stackrel{(5.3)}{=}\{\varepsilon\} \cup\left\{n u \mid n \in \mathbb{N}_{>0}, u \in \mathfrak{S}_{\gamma}\right\} \cup\left\{n u^{\prime} v \mid n \in \mathbb{N}_{>0}, u^{\prime} \in \text { leaves }\left(\mathfrak{S}_{\gamma}\right), v \in \mathfrak{S}_{\omega^{i} \cdot n}\right\} \\
& \stackrel{(5.4)}{=}\{\varepsilon\} \cup\left\{n w \mid n \in \mathbb{N}_{>0}, w \in \mathfrak{S}_{\omega^{i} \cdot n+\gamma}\right\}=\mathfrak{S}_{\omega^{i} \cdot n+\gamma+1}=\mathfrak{S}_{\omega^{i} \cdot n+\alpha} .
\end{aligned}
$$

Finally, assume that $\alpha \leq \omega^{i}$ is a limit ordinal with the fundamental sequence $\left(\alpha_{k}\right)_{k \geq 1}$. Then $\left(\omega^{i} \cdot n+\alpha_{k}\right)_{k \geq 1}$ is our fundamental sequence for the ordinal $\omega^{i} \cdot n+\alpha$. We have

$$
\mathfrak{S}_{\alpha}=\{\varepsilon\} \cup\left\{k u \mid k \in \mathbb{N}_{>0}, u \in \mathfrak{S}_{\alpha_{k}}\right\} .
$$

By induction hypothesis, for every $k \geq 1$ we have

$$
\mathfrak{S}_{\alpha_{k}} \cup\left\{u v \mid u \in \operatorname{leaves}\left(\mathfrak{S}_{\alpha_{k}}\right), v \in \mathfrak{S}_{\omega^{i} \cdot n}\right\}=\mathfrak{S}_{\omega^{i} \cdot n+\alpha_{k}} .
$$

Hence, we get

$$
\begin{aligned}
& \mathfrak{S}_{\alpha} \cup\left\{u v \mid u \in \text { leaves }\left(\mathfrak{S}_{\alpha}\right), v \in \mathfrak{S}_{\omega^{i} \cdot n}\right\} \\
& \stackrel{(5.5)}{=}\{\varepsilon\} \cup\left\{k u \mid k \in \mathbb{N}_{>0}, u \in \mathfrak{S}_{\alpha_{k}}\right\} \cup\left\{k u^{\prime} v \mid k \in \mathbb{N}_{>0}, u^{\prime} \in \operatorname{leaves}\left(\mathfrak{S}_{\alpha_{k}}\right), v \in \mathfrak{S}_{\omega^{i} \cdot n}\right\} \\
& \stackrel{(5.6)}{=}\{\varepsilon\} \cup\left\{k w \mid k \in \mathbb{N}_{>0}, w \in \mathfrak{S}_{\omega^{i} \cdot n+\alpha_{k}}\right\}=\mathfrak{S}_{\omega^{i} \cdot n+\alpha} .
\end{aligned}
$$

Now we can prove tree-automaticity of $\mathfrak{S}_{\omega^{i}}$.

Lemma 5.3. For every $i \geq 1$, the tree $\mathfrak{S}_{\omega^{i}} \subseteq \mathbb{N}_{>0}^{*}$ is tree-automatic. Moreover, there is a unary tree-automatic copy $(L, \leq)$ of $\mathfrak{S}_{\omega^{i}}$ together with a computable isomorphism $f: \mathfrak{S}_{\omega^{i}} \rightarrow(L, \leq)$.

Proof. We prove the lemma by induction on $i$. Assume that we have already constructed a tree-automatic copy $(L, \leq)$ of the tree $\mathfrak{S}_{\omega^{i}}$ over a unary alphabet (i.e., $L \subseteq \mathcal{T}_{2}^{\text {fin }}$ is regular) together with the computable isomorphism $f$. In addition, we assume that the root of $(L, \leq)$ is the one-node tree $\{\varepsilon\}$; this property is preserved by the construction. We aim at constructing a unary tree-automatic copy of $\mathfrak{S}_{\omega^{i+1}}$ with root $\{\varepsilon\}$. Let us first construct a tree-automatic copy of the computable forest $\bigsqcup_{n \geq 1} \mathfrak{S}_{\omega^{i} \cdot n}$. This forest is isomorphic to the inverse prefix relation on the domain

$$
\left\{n u \mid n \geq 1, u \in \mathfrak{S}_{\omega^{i} \cdot n}\right\} \subseteq \mathbb{N}_{>0}^{*} .
$$

Define well-founded trees $\mathfrak{T}_{n}$ for $n \geq 1$ inductively as follows. Let $\mathfrak{T}_{1}=\mathfrak{S}_{\omega^{i}}$ and let $\mathfrak{T}_{n+1}$ result from the tree $\mathfrak{S}_{\omega^{i}}$ by replacing every leaf by a copy of the tree $\mathfrak{T}_{n}$. Formally, we define

$$
\mathfrak{T}_{n}=\left\{u_{1} \cdots u_{j} v \mid 0 \leq j<n, u_{1}, \ldots, u_{j} \in \operatorname{leaves}\left(\mathfrak{S}_{\omega^{i}}\right), v \in \mathfrak{S}_{\omega^{i}}\right\} .
$$

Lemma 5.2 implies $\mathfrak{T}_{n}=\mathfrak{S}_{\omega^{i} \cdot n}$ for $n \geq 1$. We construct a tree-automatic copy of $\bigsqcup_{n \geq 1} \mathfrak{T}_{n}$ using $(L, \leq)$. The universe of this copy is the set $L^{\prime}$ of all trees of the form $\operatorname{pref}\left(\bigcup_{i=1}^{n} 0^{i} 1 t_{i}\right)$, where $n \geq 1, t_{1}, \ldots, t_{n} \in L$ and there exists $1 \leq j \leq n$ such that $t_{j}$ is a leaf of $(L, \leq)$ for all $j<i$ and $t_{j}=\{\varepsilon\}$ for all $j>i$. Since the set of leaves of $(L, \leq)$ is regular, the set $L^{\prime}$ is clearly regular. We define a tree-automatic partial order $\leq^{\prime}$ on the set $L^{\prime}$ by comparing the $t_{i}$ componentwise. Let $t=\operatorname{pref}\left(\bigcup_{i=1}^{m} 0^{i} 1 t_{i}\right) \in L^{\prime}$ and $t^{\prime}=\overline{\operatorname{pref}}\left(\bigcup_{i=1}^{n} 0^{i} 1 t_{i}^{\prime}\right) \in L^{\prime}$. Then $t \leq^{\prime} t^{\prime}$ 
if and only if $n=m$ and $t_{i} \leq t_{i}^{\prime}$ for all $1 \leq i \leq n$. From this construction, it follows easily that

$$
\left(L^{\prime}, \leq^{\prime}\right) \cong \bigsqcup_{n \geq 1} \mathfrak{T}_{n}=\bigsqcup_{n \geq 1} \mathfrak{S}_{\omega^{i} \cdot n}
$$

The set of roots of this forest is $\left\{\operatorname{pref}\left(\bigcup_{i=1}^{n} 0^{i} 1\right) \mid n \geq 1\right\}$. Let us also define a computable isomorphism $f^{\prime}: \bigsqcup_{n \geq 1} \mathfrak{T}_{n} \rightarrow\left(L^{\prime}, \leq^{\prime}\right)$. Take an element $n w$ from $\bigsqcup_{n \geq 1} \mathfrak{T}_{n}$, where $n \in \mathbb{N}_{>0}$ and $w \in \mathfrak{T}_{n}$. There is a unique factorization $w=u_{1} \cdots u_{j} v$ with $0 \leq j<n$, $u_{1}, \ldots, u_{j} \in$ leaves $\left(\mathfrak{S}_{\omega^{i}}\right)$, and $v \in \mathfrak{S}_{\omega^{i}}$. Since the set leaves $\left(\mathfrak{S}_{\omega^{i}}\right)$ is computable, we can compute this factorization. Next, using the computable isomorphism $f: \mathfrak{S}_{\omega^{i}} \rightarrow(L, \leq)$ define the trees $t_{k}=f\left(u_{k}\right)(1 \leq k \leq j), t_{j+1}=f(v)$, and $t_{j+2}, \ldots, t_{n}=\{\varepsilon\}$. Then set $f^{\prime}(n w)=\operatorname{pref}\left(\bigcup_{i=1}^{n} 0^{i} 1 t_{i}\right)$. It is straightforward to verify that this defines indeed an isomorphism from $\bigsqcup_{n>1} \mathfrak{T}_{n}$ to $\left(L^{\prime}, \leq^{\prime}\right)$.

We derive from $\left(\bar{L}^{\prime}, \leq^{\prime}\right)$ a tree-automatic copy of the computable forest $\bigsqcup_{n \geq 1} \mathfrak{S}_{\omega^{i} \cdot n+1}$. This forest is isomorphic to the inverse prefix relation on

$$
\left\{n u \mid n \in \mathbb{N}_{>0}, u \in \mathfrak{S}_{\omega^{i} \cdot n+1}\right\}=\mathbb{N}_{>0} \cup\left\{n m w \mid n, m \in \mathbb{N}_{>0}, w \in \mathfrak{S}_{\omega^{i} \cdot n}\right\} .
$$

Note that in every node $u \in \mathcal{T}_{2}^{\text {fin }}$ of the forest $\left(L^{\prime}, \leq^{\prime}\right)$, the root of $u$ has no right child (i.e., $1 \notin u)$. Define the regular set of trees

$$
L^{\prime \prime}=\left\{\operatorname{pref}\left(0^{n}\right) \mid n \geq 1\right\} \cup\left\{\operatorname{pref}\left(\left\{1^{m}\right\} \cup t\right) \mid t \in L^{\prime}, m \geq 1\right\} .
$$

On the set $L^{\prime \prime}$ we define the partial order $\leq^{\prime \prime}$ as follows. For $u, v \in L^{\prime \prime}$, let $u \leq$ " $v$ if and only if either

- $u=\operatorname{pref}\left(\left\{1^{m}\right\} \cup s\right), v=\operatorname{pref}\left(\left\{1^{m}\right\} \cup t\right)$ with $s, t \in L^{\prime}$ and $s \leq^{\prime} t$ or

- $u=\operatorname{pref}\left(\left\{1^{m}\right\} \cup t\right), v=\operatorname{pref}\left(0^{n}\right)$ with $t \in L^{\prime}$ of the form $\operatorname{pref}\left(\bigcup_{i=1}^{n} 0^{i} 1 t_{i}\right)$.

This order relation is clearly tree-automatic. Moreover, the construction implies that

$$
\left(L^{\prime \prime}, \leq^{\prime \prime}\right) \cong\left(\mathbb{N}_{>0} \cup\left\{n m w \mid n, m \in \mathbb{N}_{>0}, w \in \mathfrak{S}_{\omega^{i} \cdot n}\right\}, \succeq\right)=\bigsqcup_{n \geq 1} \mathfrak{S}_{\omega^{i} \cdot n+1}
$$

A computable isomorphism $f^{\prime \prime}: \bigsqcup_{n \geq 1} \mathfrak{S}_{\omega^{i} \cdot n+1} \rightarrow\left(L^{\prime \prime}, \leq^{\prime \prime}\right)$ can be defined as follows. For a root $n \in \mathbb{N}_{>0}$ let $f^{\prime \prime}(n)=\operatorname{pref}\left(0^{n}\right)$. A node $n m u$ with $n, m \in \mathbb{N}_{>0}$ and $u \in \mathfrak{S}_{\omega^{i} \cdot n}$ (hence, $\left.m u \in \mathfrak{S}_{\omega^{i} \cdot n+1}\right)$ is mapped to $f^{\prime \prime}(n m u)=\operatorname{pref}\left(\left\{1^{m}\right\} \cup t\right)$ with $f^{\prime}(n u)=t$.

Finally, we add to the forest $\left(L^{\prime \prime}, \leq^{\prime \prime}\right)$ the root $\{\varepsilon\}$; this gives us a tree-automatic copy of the tree $\mathfrak{S}_{\omega^{i+1}}$ with root $\{\varepsilon\}$. A computable isomorphism is obtained by extending $f^{\prime \prime}$ by $f^{\prime \prime}(\varepsilon)=\{\varepsilon\}$.

5.3. Encoding $\Sigma_{2}^{0}$-sets of binary trees. Theorem 5.1 and Lemma 5.3 show that the isomorphism problem for the following class of computable structures is $\Pi_{\omega^{i}}^{0}$-hard for every $i \in \mathbb{N}$ : the class contains all structures of the form $(V, \sqsubseteq, X)$ where $(V, \sqsubseteq)$ is the unary treeautomatic copy of $\mathfrak{S}_{\omega^{i}}$ from Lemma 5.3 and $X$ is a computable unary predicate, which is moreover a subset of leaves $(V$, $)$. By Lemma 2.12 we can moreover assume that $V \subseteq \mathcal{T}_{\text {bin }}$, i.e., $V$ consists of unlabeled full binary trees.

Let us define the set

$$
\mathcal{T}_{\text {lef }}=\left\{\{\varepsilon\} \cup 0 u \mid u \in \mathcal{T}_{\text {bin }}\right\} .
$$

Thus, $\mathcal{T}_{\text {lef }}$ contains all trees $t \in \mathcal{T}_{2}^{\text {fin }}$, where the root of $t$ has no right child $(1 \notin t)$, the root has a left child $(0 \in t)$, and the subtree rooted at 0 belongs to $\mathcal{T}_{\text {bin }}$, i.e., is a full binary tree. 
In this section, we describe an encoding of $\Sigma_{2}^{0}$-subsets of $\mathcal{T}_{\text {lef }}$ by sets of tree-automatic trees of height 3 . Actually, we need this encoding only for computable subsets of $\mathcal{T}_{\text {lef }}$ (instead of $\Sigma_{2}^{0}$-sets), but the proof of Lemma 5.4 is not simpler for a computable set $B$.

Lemma 5.4. There are two trees $\mathfrak{U}_{0}$ and $\mathfrak{U}_{1}$ of height $3\left(\mathfrak{U}_{0} \neq \mathfrak{U}_{1}\right)$ with the following property: From a given index of a $\Sigma_{2}^{0}$-set $B \subseteq \mathcal{T}_{\text {lef }}$ one can effectively construct a treeautomatic forest $\mathfrak{F}_{B}$ of height 3 such that:

- The set of roots of $\mathfrak{F}_{B}$ is $\mathcal{T}_{\text {lef. }}$.

- For every $t \in \mathcal{T}_{\text {lef }}, \mathfrak{F}_{B}(t) \cong \mathfrak{U}_{0}$ if $t \in B$ and $\mathfrak{F}_{B}(t) \cong \mathfrak{U}_{1}$ if $t \notin B$.

Restricting to trees from $\mathcal{T}_{\text {lef }}$ makes our encoding technically a bit simpler, and this restriction can be easily enforced later when we apply Lemma 5.4.

We prove Lemma 5.4 using a similar statement for words from [20]. First, we have to introduce a notation from [20]. Let $\mathcal{A}=(Q, \Sigma, \Delta, I, F)$ be a finite nondeterministic string automaton, where $Q$ is the set of states, $\Sigma$ is the input alphabet, $\Delta \subseteq Q \times \Sigma \times Q$ is the set of transition triples, $I$ is the set of initial states, and $F$ is the set of final states. A successful run of $\mathcal{A}$ on a non-empty word $w \in \Sigma^{+}$is a word $\left(q_{0}, a_{1}, q_{1}\right)\left(q_{1}, a_{2}, q_{2}\right) \cdots\left(q_{n-1}, a_{n}, q_{n}\right)$ over $\Delta$ such that $q_{0} \in I, q_{n} \in F$, and $w=a_{1} a_{2} \cdots a_{n}$. The language $L(\mathcal{A})$ accepted by $\mathcal{A}$ consists of all non-empty words (for technical reasons, the empty word was excluded in [20]) that have a successful run. We define a forest forest $(\mathcal{A})$ in the following. Clearly, $(L(\mathcal{A}), \succeq)$ (recall that $\preceq$ is the prefix relation on words) is a (string-automatic) forest. The set of all leaves of the forest $(L(\mathcal{A}), \succeq)$ is first-order definable whence it is a regular language. It is the set of all words in $L(\mathcal{A})$ that are not a proper prefix of another word in $(L(\mathcal{A})$. Let us denote the set of leaves of $(L(\mathcal{A}), \succeq)$ with leaves $(\mathcal{A})$. We define the forest forest $(\mathcal{A})$ as follows:

- The domain of forest $(\mathcal{A})$ is the regular set

$$
L(\mathcal{A}) \sqcup\left\{r \in \Delta^{+} \mid r \text { is a successful run of } \mathcal{A} \text { on some } v \in \text { leaves }(\mathcal{A})\right\} .
$$

- The order relation $\leq$ of forest $(\mathcal{A})$ is defined by $u \leq v$ if

(1) either $u, v \in L(\mathcal{A})$ and $v \preceq u$ or

(2) $v \in L(\mathcal{A})$ and $u \in \Delta^{+}$is a successful run of $\mathcal{A}$ on some $w \in \operatorname{leaves}(\mathcal{A})$ with $v \preceq w$.

Clearly, forest $(\mathcal{A})$ is string-automatic. Intuitively, we take the forest resulting from the inverse prefix order on the regular language $L(\mathcal{A})$ and append to each leaf $v$ of $(L(\mathcal{A}), \succeq)$ all successful runs of $\mathcal{A}$ on $v$ as children. All these children are leaves in forest $(\mathcal{A})$. In [20], the following lemma was proved.

Lemma 5.5. There exist two trees $\mathfrak{U}_{0}$ and $\mathfrak{U}_{1}$ of height $3\left(\mathfrak{U}_{0} \neq \mathfrak{U}_{1}\right)$ with the following property: From a given index of a $\Sigma_{2}^{0}$-set $A \subseteq\{0,1\}^{*} 1$ one can effectively construct a finite string automaton $\mathcal{A}$ (over an alphabet $\Sigma$ with $0,1, \sharp \in \Sigma$ ) such that forest $(\mathcal{A})$ is a forest of height 3 with the following properties.

- The set of roots of forest $(\mathcal{A})$ is $\{0,1\}^{*} 1 \sharp$.

- For every $w \in\{0,1\}^{*} 1$, forest $(\mathcal{A})(w \sharp) \cong \mathfrak{U}_{0}$ if $w \in A$, and forest $(\mathcal{A})(w \sharp) \cong \mathfrak{U}_{1}$ if $w \notin A$.

In order to prove Lemma 5.4 using Lemma 5.5, we have to encode trees from $\mathcal{T}_{\text {lef }}$ by words. A tree $t \in \mathcal{T}_{\text {bin }}$ can be encoded by a non-empty bracket expression, i.e., a word over the alphabet $\{()$,$\} . Here, we view such a bracket expression as a binary string by$ identifying ( with 0 and ) with 1 . Thus, we define a nonempty word word $(t) \in\{0,1\}^{+}$as follows: Consider a depth-first left-to-right traversal of $t$. Each time, we move from a node $v$ to one of its children, we write down 0 . Each time, we move from a node $v i$ to its parent 
node $v$, we write down 1 . The resulting word is word $(t)$. Formally, let word $(\{\varepsilon\})=\varepsilon$ and for $t \in \mathcal{T}_{\text {bin }} \backslash\{\{\varepsilon\}\}$ such that $t=\{\varepsilon\} \cup 0 t_{1} \cup 1 t_{2}$ let

$$
\operatorname{word}(t)=0 \operatorname{word}\left(t_{1}\right) 10 \text { word }\left(t_{2}\right) 1 \text {. }
$$

This mapping word is clearly injective. Finally, for $t=(\{\varepsilon\} \cup 0 u) \in \mathcal{T}_{\text {lef }}$ with $u \in \mathcal{T}_{\text {bin }}$ let $\operatorname{word}(t)=0$ word $(u) 1$. Also the mapping word : $\mathcal{T}_{\text {lef }} \rightarrow 0\{0,1\}^{*} 1$ is injective.

Let us now fix an alphabet $\Sigma$ such that $0,1, \sharp \in \Sigma$ (as in Lemma 5.5). Take a word $w=u \sharp v$ with $u \in\{0,1\}^{*}, v \in \Sigma^{*}$ and $u=\operatorname{word}(t)$ for some tree $t \in \mathcal{T}_{\text {lef }}$. Note that the root of $t$ has no right child in $t$. For $1 \leq i \leq|v|$ let $a_{i}$ be the $i^{t h}$ symbol of $v$. We encode $w$ by the $\Sigma$-labeled tree tree $(w)=(T, \lambda) \in \mathcal{T}_{2, \Sigma}^{\text {fin }}$, where

$$
\begin{aligned}
T & =t \cup\left\{1^{i}|1 \leq i \leq| v \mid\right\} \text { and } \\
\lambda(x) & = \begin{cases}a_{i} & \text { if } x=1^{i} \text { for some } 1 \leq i \leq|v|, \\
\sharp & \text { else. }\end{cases}
\end{aligned}
$$

Note that tree $(\operatorname{word}(t) \sharp)=t$, since we identify an unlabeled tree with a tree where all nodes are labeled with $\sharp$.

Lemma 5.6. From a given string automaton $\mathcal{A}$ over $\Sigma$ one can construct effectively a tree automaton $\mathcal{B}$ over $\Sigma$ such that for every tree $t \in \mathcal{T}_{\text {lef }}$ and every word $v \in \Sigma^{*}$ the following holds: The number of successful runs of $\mathcal{A}$ on the string word $(t) \sharp v$ equals the number of successful runs of $\mathcal{B}$ on the tree tree $(\operatorname{word}(t) \sharp v)$.

Proof. Let $\mathcal{A}=(Q, \Sigma, \Delta, I, F)$. Note that tree $(\operatorname{word}(t) \sharp v)$ consists of the tree $t$ to which we add at the root a right branch of length $|v|$. The $i^{t h}$ node on this branch is labeled with the $i^{t h}$ symbol of $\sharp v$ (we count from the root to the leaf). Essentially, the tree automaton $\mathcal{B}$ simulates a tree-walking automaton $\mathcal{W}$ (see [5] for a survey on tree walking automata, but we do not need a formal definition of tree walking automata) that walks over the tree tree $($ word $(t) \$ v)$ in depth-first left-to-right order. Thereby, $\mathcal{W}$ simulates the string automaton $\mathcal{A}$. The automaton $\mathcal{W}$ starts in the root of the tree. In a first phase (which is finished if $\mathcal{W}$ returns to the root for the first time), $\mathcal{W}$ behaves as follows: each time, $\mathcal{W}$ moves down in the tree (towards the leaves), it simulates a 0 -labeled transition of $\mathcal{A}$, and each time, $\mathcal{W}$ moves up in the tree (towards the root), it simulates a 1-labeled transition of $\mathcal{A}$. After the first phase, the tree $t$ is fully traversed and $\mathcal{W}$ goes into the right branch of tree $(\operatorname{word}(t) \sharp v)$ (which is labeled with the word $\sharp v$ ) and continues the simulation of $\mathcal{A}$.

Here is a formal definition of the tree automaton $\mathcal{B}=\left(Q^{\prime}, \Delta^{\prime}, I^{\prime}, F^{\prime}\right)$, which simulates a tree-walking automaton $\mathcal{W}$ with the above behavior. Fix an arbitrary final state $q_{\mathrm{f}} \in F$. The state set of $\mathcal{B}$ is

$$
Q^{\prime}=(Q \times Q \times Q) \cup(I \times Q) \cup Q
$$

the set of initial states is $I^{\prime}=I \times Q$, and the set of final states is $F$. The set of transitions is $\Delta^{\prime}=\Delta_{1} \cup \Delta_{2} \cup \Delta_{3}$, where

$$
\begin{aligned}
\Delta_{1}= & \left\{\left(\left(p_{1}, p_{2}\right), \sharp,\left(q_{1}, q_{2}, q_{3}\right), r\right) \mid p_{1} \in I,\left(p_{1}, 0, q_{1}\right) \in \Delta, q_{2} \in Q,\left(q_{3}, 1, p_{2}\right),\left(p_{2}, \sharp, r\right) \in \Delta\right\}, \\
\Delta_{2}= & \left\{\left(\left(p_{1}, p_{2}, p_{3}\right), \sharp,\left(q_{1}, q_{2}, q_{3}\right),\left(r_{1}, r_{2}, r_{3}\right)\right) \mid\right. \\
& \left.\left(p_{1}, 0, q_{1}\right), q_{2} \in Q,\left(q_{3}, 1, p_{2}\right),\left(p_{2}, 0, r_{1}\right), r_{2} \in Q,\left(r_{3}, 1, p_{3}\right) \in \Delta\right\} \\
\cup & \left\{\left((p, p, p), \sharp, q_{\mathrm{f}}, q_{\mathrm{f}}\right) \mid p \in Q\right\}, \\
\Delta_{3}= & \left\{\left(p, a, q_{\mathrm{f}}, q\right) \mid(p, a, q) \in \Delta\right\} .
\end{aligned}
$$


With the transitions in $\Delta_{1}$ we split the simulation of the tree-walking automaton into its first and second phase, i.e., $p_{2}$ in $\Delta_{1}$ is the state reached by the tree-walking automaton after traversing the tree $t$. The transitions in $\Delta_{2}$ simulate the traversal of $t$, whereas the transitions in $\Delta_{3}$ simulate the the string automaton $\mathcal{A}$ on the right $\sharp v$-labeled branch.

Let us now prove Lemma 5.4 .

Proof of Lemma 5.4. Fix a $\Sigma_{2}^{0}$-set $B \subseteq \mathcal{T}_{\text {lef }}$. Then the set $A=\operatorname{word}(B) \subseteq 0\{0,1\}^{*} 1$ belongs to $\Sigma_{2}^{0}$ as well (the range of the word-mapping is computable and on its range, the inverse of word is also computable). Therefore, we can apply Lemma 5.5 to the set $\operatorname{word}(B)$. We obtain (effectively) a finite string automaton $\mathcal{A}$ (over an alphabet $\Sigma$ with $0,1, \sharp \in \Sigma$ ) such that forest $(\mathcal{A})$ is a forest of height 3 with the following properties.

- The set of roots of forest $(\mathcal{A})$ is $\{0,1\}^{*} 1 \sharp$.

- For every $w \in\{0,1\}^{*} 1$, forest $(\mathcal{A})(w \sharp) \cong \mathfrak{U}_{0}$ if $w \in A$, and forest $(\mathcal{A})(w \sharp) \cong \mathfrak{U}_{1}$ if $w \notin A$ for two nonisomorphic trees $\mathfrak{U}_{0}$ and $\mathfrak{U}_{1}$.

To the string automaton $\mathcal{A}$ we next apply Lemma 5.6. We obtain (effectively) a tree automaton $\mathcal{B}$ over $\Sigma$ such that for every tree $t \in \mathcal{T}_{\text {lef }}$ and every word $v \in \Sigma^{*}$ the following holds: The number of successful runs of $\mathcal{A}$ on the string word $(t) \sharp v$ equals the number of successful runs of $\mathcal{B}$ on the tree tree $($ word $(t) \sharp v)$. Since $\mathcal{A}$ accepts every word from $\{0,1\}^{*} 1 \sharp$ (this set is the set of roots of forest $(\mathcal{A})$ ), $\mathcal{B}$ accepts every tree $t \in \mathcal{T}_{\text {lef }}$.

By taking the product with a deterministic tree automaton that accepts the set of trees

$$
\left\{\operatorname{tree}(\operatorname{word}(t) \sharp v) \mid t \in \mathcal{T}_{\text {lef }}, v \in \Sigma^{*}\right\}
$$

(which is regular), we can assume that

$$
L(\mathcal{B}) \subseteq\left\{\operatorname{tree}(\operatorname{word}(t) \sharp v) \mid t \in \mathcal{T}_{\text {lef }}, v \in \Sigma^{*}\right\} .
$$

For trees $t_{1}=\operatorname{tree}\left(\operatorname{word}(t) \sharp v_{1}\right), t_{2}=\operatorname{tree}\left(\operatorname{word}(t) \sharp v_{2}\right)$ let us write $t_{1} \sqsubseteq t_{2}$ if $v_{1}$ is a prefix of $v_{2}$. Clearly, this is a tree-automatic relation. Let

$$
\max (\mathcal{B})=\left\{t \in L(\mathcal{B}) \mid \text { there does not exist } t^{\prime} \in L(\mathcal{B}) \text { with } t \sqsubset t^{\prime}\right\} ;
$$

this set is regular as well. We can now construct a tree-automatic forest $\mathfrak{F}_{B}$ of height 3 as follows. The set of nodes of $\mathfrak{F}_{B}$ is

$$
L(\mathcal{B}) \cup \bigcup_{t \in \max (\mathcal{B})} \operatorname{Run}(\mathcal{B}, t) .
$$

Since $\max (\mathcal{B})$ is regular, this set is also regular. The order relation of the forest $\mathfrak{F}_{B}$ is the tree-automatic relation

$$
\sqsupseteq \cup\{(\rho, t) \mid t \in L(\mathcal{B}), \exists u \in \max (\mathcal{B}): t \sqsubseteq u, \rho \in \operatorname{Run}(\mathcal{B}, u)\} .
$$

The set of roots of $\mathfrak{F}_{B}$ is (as required) $\mathcal{T}_{\text {lef }}$. Moreover, for every tree $t \in \mathcal{T}_{\text {lef }}$, the construction directly implies that $\mathfrak{F}_{B}(t) \cong \operatorname{forest}(\mathcal{A})($ word $(t) \sharp)$. Hence, for every tree $t \in \mathcal{T}_{\text {lef }}$ we have $\mathfrak{F}_{B}(t) \cong \mathfrak{U}_{0}$ if and only if forest $(\mathcal{A})(\operatorname{word}(t) \sharp) \cong \mathfrak{U}_{0}$ if and only if $\operatorname{word}(t) \in A=\operatorname{word}(B)$ if and only if $t \in B$, and similarly, $\mathfrak{F}_{B}(t) \cong \mathfrak{U}_{1}$ if and only if $t \notin B$. 
5.4. Hardness for the isomorphism problem. Hardness of the isomorphism problem for well-founded tree-automatic trees is established through the following theorem.

Theorem 5.7. From a given $i \in \mathbb{N}_{>0}$, one can compute a well-founded tree-automatic tree $\mathfrak{V}_{i}$ such that the following holds: From a given $\Pi_{\omega^{i}}^{0}$-set $P \subseteq \mathbb{N}$ (represented by a $\Pi_{\omega^{i}}^{0}$ index) and $n \in \mathbb{N}$ one can compute a well-founded tree-automatic tree $\mathfrak{W}_{P, n}$ such that $n \in P$ if and only if $\mathfrak{V}_{i} \cong \mathfrak{W}_{P, n}$

Proof. Fix $i \geq 1$, an arbitrary $\Pi_{\omega^{i}}^{0}$-set $P \subseteq \mathbb{N}$, and $n \in \mathbb{N}$. According to Theorem 5.1 there exists effectively a computable subset $L_{i}=L_{\infty}^{\omega^{i}} \subseteq$ leaves $\left(\mathfrak{S}_{\omega^{i}}\right)$ such that the following holds. From an index for $P$ and $n$ one can compute an index for a computable subset $T_{P, n} \subseteq$ leaves $\left(\mathfrak{S}_{\omega^{i}}\right)$ such that $\left(\mathfrak{S}_{\omega^{i}}, L_{i}\right) \cong\left(\mathfrak{S}_{\omega^{i}}, T_{P, n}\right)$ if and only if $n \in P$. By Lemma 5.3 , the tree $\mathfrak{S}_{\omega^{i}}$ is tree-automatic and there exists a unary tree-automatic copy $(S, \leq)$ of $\mathfrak{S}_{\omega^{i}}$ together with a computable isomorphism $f: \mathfrak{S}_{\omega^{i}} \rightarrow(S, \leq)$. Moreover, by Lemma 2.12 we can assume that $S \subseteq \mathcal{T}_{\text {bin }}$. Finally, by applying the computable bijection $t \mapsto\{\varepsilon\} \cup 0 t$ from $\mathcal{T}_{\text {bin }}$ to $\mathcal{T}_{\text {lef }}$, we can even assume that $S \subseteq \mathcal{T}_{\text {lef }}$. Since $f$ is computable and bijective and $L_{i}$ and $T_{P, n}$ are computable, the sets $L_{i}^{\prime}=f\left(L_{i}\right) \subseteq S$ and $T_{P, n}^{\prime}=f\left(T_{P, n}\right) \subseteq S$ are computable as well and in particular $\Sigma_{2}^{0}$. We have $n \in P$ if and only if $\left(S, \leq, L_{i}^{\prime}\right) \cong\left(S, \leq, T_{P, n}^{\prime}\right)$.

Next, using Lemma 5.4 we obtain two trees $\mathfrak{U}_{0}$ and $\mathfrak{U}_{1}$ of height 3 and from the indices of the computable sets $L_{i}^{\prime}$ and $T_{P, n}^{\prime}$, we can compute two tree-automatic forests $\mathfrak{G}_{i}$ and $\mathfrak{H}_{P, n}$ of height 3 such that the following holds.

- The set of roots of $\mathfrak{G}_{i}\left(\right.$ resp. $\mathfrak{H}_{P, n}$ ) is $\mathcal{T}_{\text {lef }}$.

- For every $t \in \mathcal{T}_{\text {lef }}, \mathfrak{G}_{i}(t) \cong \mathfrak{U}_{0}$ if $t \in L_{i}^{\prime}$ and $\mathfrak{G}_{i}(t) \cong \mathfrak{U}_{1}$ otherwise.

- For every $t \in \mathcal{T}_{\text {lef }}, \mathfrak{H}_{P, n}(t) \cong \mathfrak{U}_{0}$ if $t \in T_{P, n}^{\prime}$ and $\mathfrak{H}_{P, n}(t) \cong \mathfrak{U}_{1}$ otherwise.

Note that by Theorem 2.13, leaves $(S, \leq)$ is a regular set of trees since it is first-order definable in the tree-automatic tree $(S, \leq)$. Let $\mathfrak{G}_{i}^{\prime}\left(\operatorname{resp} . \mathfrak{H}_{P, n}^{\prime}\right)$ be the restriction of the forest $\mathfrak{G}_{i}$ (resp. $\left.\mathfrak{H}_{P, n}\right)$ to those trees with a root from leaves $(S, \leq)$. It follows that $\mathfrak{G}_{i}^{\prime}$ and $\mathfrak{H}_{P, n}^{\prime}$ are again tree-automatic forests of height 3. Moreover, we can assume that the intersection of the domains of $\mathfrak{G}_{i}^{\prime}$ (resp. $\left.\mathfrak{H}_{P, n}^{\prime}\right)$ and $S$ equals leaves $(S, \leq)$. Finally, let $\mathfrak{V}_{i}$ (resp. $\mathfrak{W}_{P, n}$ ) be the well-founded tree-automatic tree obtained from the union of $\mathfrak{G}_{i}^{\prime}$ (resp. $\mathfrak{H}_{P, n}^{\prime}$ ) and $(S, \leq)$. Hence, $\mathfrak{V}_{i}$ (resp. $\left.\mathfrak{W}_{P, n}\right)$ results from the tree $(S, \leq)$ by (i) replacing every leaf which belongs to $L_{i}^{\prime}$ (resp. $T_{P, n}^{\prime}$ ) by the tree-automatic height-3 tree $\mathfrak{U}_{0}$ and by (ii) replacing every leaf which does not belong to $L_{i}^{\prime}$ (resp. $T_{P, n}^{\prime}$ ) by the tree-automatic height-3 tree $\mathfrak{U}_{1}$. Since $\mathfrak{U}_{0} \not \mathfrak{U}_{1}$, we have $n \in P$ if and only if $\left(S, \leq, L_{i}^{\prime}\right) \cong\left(S, \leq, T_{P, n}^{\prime}\right)$ if and only if $\mathfrak{V}_{i} \cong \mathfrak{W}_{P, n}$.

Theorem 5.8. The isomorphism problem for well-founded tree-automatic trees is $\Delta_{\omega^{\omega}}^{0}$-hard under Turing-reductions.

Proof. Let $\Phi_{e}$ be a total computable function that maps $i \in \mathbb{N}$ to an ordinal notation $a_{i} \in O$ with $\left|a_{i}\right|_{O}=\omega^{i}$. Hence $3 \cdot 5^{e}$ is an ordinal notation for $\omega^{\omega}$. Recall that $\Delta_{\omega^{\omega}}^{0}$ consists of all sets that are Turing-reducible to

$$
H\left(3 \cdot 5^{e}\right)=\left\{\langle a, n\rangle \mid a<_{O} 3 \cdot 5^{e}, n \in H(a)\right\} .
$$

This set is Turing-reducible to

$$
A=\left\{\langle i, n\rangle \mid i \geq 1, n \in H\left(a_{i}\right)\right\} .
$$

To see this, take a pair $\langle a, n\rangle$. First, check whether $a<_{O} 3 \cdot 5^{e}$. Since the set $\{b \in O \mid$ $\left.b<_{O} 3 \cdot 5^{e}\right\}$ is computably enumerable [1, Prop. 4.10], this is effectively possible using the 
halting problem as an oracle. Clearly, the halting problem is computable in $H\left(a_{1}\right)=H(\omega)$ and hence in $A$. If $a<_{O} 3 \cdot 5^{e}$, we can compute effectively $i \in \mathbb{N}$ with $a<_{O} a_{i}$ : Simply enumerate all sets $B_{i}=\left\{b \in O \mid b<_{O} a_{i}\right\}$ until $a$ is found. Having $i \in \mathbb{N}$ with $a<_{O} a_{i}$ we can finally compute $m$ such that $m \in H\left(a_{i}\right)$ if and only if $n \in H(a)$ (more precisely, from $a, a_{i} \in O$ one can compute an index for a many-one reduction of $H(a)$ to $H\left(a_{i}\right)$ [24, p. 437]).

Finally, we reduce the set $A$ to the isomorphism problem for well-founded tree-automatic trees. Take a pair $\langle i, n\rangle$. From $i, n$ (and a $\Pi_{\omega^{i}}^{0}$ index for the $\Delta_{\omega^{i}}^{0}$-set $H\left(a_{i}\right)$, which can be computed from $i$ ) we can compute by Theorem 5.7 two well-founded tree-automatic trees $\mathfrak{V}$ and $\mathfrak{W}$ such that $n \in H\left(a_{i}\right)$ if and only if $\mathfrak{V} \cong \mathfrak{W}$. Hence, $\langle i, n\rangle \in A$ if and only if $\mathfrak{V} \cong \mathfrak{W}$. This proves the theorem.

\section{ACKNOWLEDGMENT}

We thank Dietrich Kuske and the anonymous referees of this paper and its conference version for their useful comments. In particular, we thank one of the referees for simplifying our arguments in Example 3.27 .

\section{REFERENCES}

[1] Christopher J. Ash and Julia F. Knight. Computable structures and the hyperarithmetical hierarchy, volume 144 of Studies in Logic and the Foundations of Mathematics. North-Holland Publishing Co., Amsterdam, 2000.

[2] Vince Bárány, Erich Grädel, and Sasha Rubin. Automata-based presentations of infinite structures. In Finite and Algorithmic Model Theory, number 379 in London Mathematical Society Lecture Notes Series, pages 1-76. Cambridge University Press, 2011.

[3] Achim Blumensath. Automatic structures. Diploma thesis, RWTH Aachen, 1999.

[4] Achim Blumensath and Erich Grädel. Finite presentations of infinite structures: Automata and interpretations. Theory of Computing Systems, 37:642-674, 2004.

[5] Mikolaj Bojanczyk. Tree-walking automata, 2008. tutorial at LATA 2008, Www.mimuw.edu.pl/ bojan/papers/twasurvey.pdf

[6] Wesley Calvert and Julia F. Knight. Classification from a computable viewpoint. Bulletin of Symbolic Logic, 12(2):191-218, 2006.

[7] Philip W. Carruth. Arithmetic of ordinals with applications to the theory of ordered Abelian groups. Bulletin of the American Mathematical Society, 48:262-271, 1942.

[8] S. Barry Cooper, Anuj Dawar, and Benedikt Löwe, editors. How the World Computes - Turing Centenary Conference and 8th Conference on Computability in Europe, CiE 2012, Cambridge, UK, June 18-23, 2012. Proceedings, volume 7318 of Lecture Notes in Computer Science. Springer, 2012.

[9] Christian Delhommé. Automaticité des ordinaux et des graphes homogènes. Comptes Rendus de l'Académie des Sciences, Series I, 339:5-10, 2004.

[10] Sergei S. Goncharov and Julia F. Knight. Computable structure and antistructure theorems. Algebra Logika, 41(6):639-681, 2002.

[11] Denis R. Hirschfeldt and Walker M. White. Realizing levels of the hyperarithmetic hierarchy as degree spectra of relations on computable structures. Notre Dame Journal of Formal Logic, 43(1):51-64, 2002.

[12] Martin Huschenbett. Word automaticity of tree automatic scattered linear orderings is decidable. In Cooper et al. 8], pages 313-322.

[13] Alexander Kartzow. First-Order Model Checking On Generalisations of Pushdown Graphs. PhD thesis, TU Darmstadt Fachbereich Mathematik, July 2011.

[14] Alexander Kartzow, Jiamou Liu, and Markus Lohrey. Tree-automatic well-founded trees. In Cooper et al. [8], pages 363-373. 
[15] Alexander Kartzow, Jiamou Liu, and Markus Lohrey. Tree-automatic well-founded trees. CoRR, abs/1201.5495, 2012. Version 1.

[16] Bakhadyr Khoussainov and Mia Minnes. Model theoretic complexity of automatic structures. Annals of Pure and Applied Logic, 161(3):416-426, 2009.

[17] Bakhadyr Khoussainov and Anil Nerode. Automatic presentations of structures. In LCC: International Workshop on Logic and Computational Complexity, number 960 in Lecture Notes in Computer Science, pages 367-392. Springer, 1995.

[18] Bakhadyr Khoussainov, André Nies, Sasha Rubin, and Frank Stephan. Automatic structures: richness and limitations. Logical Methods in Computer Science, 3(2):2:2, 18 pp. (electronic), 2007.

[19] Bakhadyr Khoussainov, Sasha Rubin, and Frank Stephan. Automatic linear orders and trees. ACM Transactions on Computational Logic, 6(4):675-700, 2005.

[20] Dietrich Kuske, Jiamou Liu, and Markus Lohrey. The isomorphism problem on classes of automatic structures with transitive relations. to appear in Transactions of the American Mathematical Society.

[21] Dietrich Kuske and Markus Lohrey. Automatic structures of bounded degree revisited. Journal of Symbolic Logic, 76(4):1352-1380, 2011.

[22] Graham P. Oliver and Richard M. Thomas. Automatic presentations for finitely generated groups. In Volker Diekert and Bruno Durand, editors, Proceedings of STACS 2005, number 3404 in Lecture Notes in Computer Science, pages 693-704. Springer, 2005.

[23] Frank P. Ramsey. On a problem of formal logic. Proceedings of the London Mathematical Society, $2: 30(1): 264-286,1930$.

[24] Hartley Rogers. Theory of Recursive Functions and Effective Computability. McGraw-Hill, 1968.

[25] Joseph G. Rosenstein. Linear Ordering. Academic Press, 1982.

[26] Anthony W. To and L. Libkin. Recurrent reachability analysis in regular model checking. In Iliano Cervesato, Helmut Veith, and Andrei Voronkov, editors, Proceedings of LPAR 2008, number 5330 in Lecture Notes in Computer Science, pages 198-213, 2008. 